\title{
Investigation of Polymer-Filler Interactions Using Functionalized Nanoparticles
}

\author{
Dissertation \\ zur Erlangung des mathematisch-naturwissenschaftlichen Doktorgrades „Doctor \\ rerum naturalium" \\ der Georg-August-Universität Göttingen \\ im Promotionsprogramm Chemie der \\ Georg-August University School of Science (GAUSS)

\section{vorgelegt von \\ Annika Nitschke \\ aus Uelzen}

Göttingen, 2020 
Betreuungsausschuss

Prof. Dr. Philipp Vana, MBA Institut für Physikalische Chemie

Georg-August-Universität Göttingen

Prof. Dr. Michael Buback

Institut für Physikalische Chemie

Georg-August-Universität Göttingen

\section{Mitglieder der Prüfungskommission}

Referent

Prof. Dr. Philipp Vana, MBA Institut für Physikalische Chemie Georg-August-Universität Göttingen

Korreferent

Prof. Dr. Michael Buback

Institut für Physikalische Chemie Georg-August-Universität Göttingen

Weitere Mitglieder der Prüfungskommission

Prof. Dr. Alec Wodtke

Prof. Dr. Burkhard Geil

Prof. Dr. Thomas Zeuch

Dr. Matthias Otte
Institut für Physikalische Chemie Georg-August-Universität Göttingen Institut für Physikalische Chemie Georg-August-Universität Göttingen Institut für Physikalische Chemie Georg-August-Universität Göttingen Institut für Anorganische Chemie Georg-August-Universität Göttingen

Tag der mündlichen Prüfung: 04.03.2020 


\section{Acknowledgments}

First of all, I want to express my profound gratitude to my supervisor Prof. Dr. Philipp Vana for his extraordinary guidance and unconditional support during the past four years. I am thankful for the great opportunity to take part in a collaboration project and thus working on an exciting and partially industry-oriented topic. Furthermore, I highly appreciate that he continuously motivated me and encouraged me to further develop myself as a scientist and as a person.

I would like to express my appreciation and a sincere thanks to my cosupervisor Prof. Dr. Michael Buback for helping me out and taking over the supervision during my thesis. I enjoyed the many inspiring and useful discussions we had. I am particularly grateful for his support and dedication to the kinetic studies, which were totally new to me when I started the project. Additionally, special thanks go to his wife for allowing him to postpone his retirement for me.

Prof. Dr. Alec Wodtke, Prof. Dr. Burkhard Geil, Prof. Dr. Thomas Zeuch, and Dr. Matthias Otte are gratefully acknowledged for being part of my thesis committee.

I want to thank the Continental Reifen $\mathrm{GmbH}$ for financing my project and especially Prof. Dr. Nils Hojdis and the members of the ATM division for the great cooperation. I did learn a lot from you and always enjoyed working together.

I consider myself very lucky to have been able to work together with a couple of gifted students, that contributed practically to this thesis: my bachelor students Laura Brinkmann and Vanessa Braun, as well as my interns Nils Gehrmann, Marc Christian Neben and Leon Kollenbach, who did research under my supervision. Thank you all for the thriving and pleasant collaboration. 
I want to thank all current and former members of the MMC and TMC groups for contributing to a very nice working environment, for a lot of support, interesting and cheerful discussions and for making the $\mathrm{PhD}$ a very joyful time. A special thanks goes to Lara Riemann for the excellent cooperation in writing the joint publication. I would also like to acknowledge Judith Steinhoff, Stella Weber, Lara Riemann and Niklas Frerichs for thorough proof reading of this work.

The past ten years in Göttingen were an extraordinary time which I would not have loved so much without the people that accompanied me. I am thankful for each one of you and for the memories we will always share. I am especially grateful to Jana Dumrese and Nikolas Wolter, who are my dearest friends and are like family to me. Thank you so much for your support in any situation of life and that I can always rely on you.

Finally, I would like to express my gratitude to my family for their unlimited support. Without you, I would not be who and where I am now. 


\section{Abstract}

Silica and carbon black (CB) particles were successfully functionalized by exploiting the characteristic features of the reversible addition-fragmentation chain transfer (RAFT) polymerization. This enabled both the contribution to a better understanding of the interactions within filled rubber compounds and the improvement of their mechanical properties.

Silica nanoparticles were functionalized with styrene butadiene rubber (SBR) via grafting-from and grafting-to approaches using various RAFT agents for the polymerization from the particle surface as well as two different strategies for the grafting of polymer to the surface. The grafting density of surface bound polymer was determined via thermogravimetric analysis (TGA) and elemental analysis (EA), revealing it's depended on the used approach and RAFT agent, respectively. The so-obtained hybrid silica nanoparticles were used as fillers in typical tire compounds and physical tests were conducted to investigate the influence of the functionalization on the interactions within the filled compounds. It was demonstrated that the mechanical properties were improved compared to the use of unfunctionalized or even silanized silica nanoparticles. This was mainly attributed to an increase of polymer-filler interactions through the entanglement of the surface bound polymer with the polymeric matrix. The results showed that the use of functionalized silica nanoparticles is a powerful tool for the adjustment and tuning of the mechanical properties of tire compounds.

A different strategy was adopted for the functionalization of CB particles applying a grafting-to approach using copolymers with anchor groups within the polymer side chain. Pyridine, furan and epoxide units were chosen as anchor groups, due to their different binding mechanisms with the $\mathrm{CB}$ surface. As material design requires the ability to precisely tune a materials properties, further investigations into the grafting conditions were conducted using methacrylate copolymers and thereby new insights into the binding mechanisms were revealed. It was found that the Diels-Alder reaction of 
the furan anchor group can only occur up to a certain amount of functional groups at the CB surface. The grafting of the epoxide anchor group via a ring opening reaction is favored by external energy input, i.e. by elevated temperatures. In contrast, the non-covalent grafting of the pyridine unit to the CB surface occurs fast, even at room temperature. Additionally, the grafting-to approach was transferable to butadiene-based copolymers. The grafting density of the respective pyridine anchor group-containing copolymer was comparable to that of the methacrylate copolymer, confirming the applicability of the grafting-to approach with the chosen anchor groups for a variety of monomer classes and materials.

Another part of this thesis was the investigation into the kinetics of $n$ pentyl methacrylate (PnMA) radical polymerization and the determination of the Mark-Houwink coefficients (MHCs) of poly(pentyl methacrylate) (PPnMA). Both have not been reported before, but the precise knowledge of rate coefficients is of key importance for the understanding and the application of radical polymerization processes and the MHCs are essential for the analysis of the molecular weight distribution (MWD) obtained from size-exclusion chromatography (SEC). For the first time, the MHCs were determined based on the principle of universal calibration from a double-log plot of SEC data obtained with poly(methyl methacrylate) calibration in combination with the calculated molecular weights of the PPnMA samples from RAFT polymerization. Propagation rate coefficients $\left(k_{\mathrm{p}}\right)$ of PnMA radical polymerization were measured in bulk and in toluene solution over an extended temperature range via pulsed laser polymerization in conjunction with SEC (PLP-SEC). The so-obtained data allows for modeling PnMA polymerization kinetics and product properties at moderate degrees of monomer conversion. The comparison with reported data for several other alkyl methacrylates showed a distinct family behavior of this group of monomers. 


\section{Contents}

1 Introduction and Motivation 1

2 Theoretical Background 3

2.1 Fundamentals of RAFT Polymerization . . . . . . . . . . 3

2.1 .1 The RAFT Mechanism . . . . . . . . . . . . 4

2.1 .2 RAFT Polymers on Surfaces . . . . . . . . . . . . 4

2.2 Fundamentals of Radical Copolymerizations . . . . . . . 7

2.3 Fundamentals of Tire Materials $\ldots \ldots \ldots \ldots$. . . . . . 8

2.3.1 Composition of Tire Materials . . . . . . . . . . 8

2.3.2 Mechanical Properties of Rubber Compounds . . . . 9

3 SBR-Functionalized Silica Nanoparticles - Grafting-from versus Grafting-to Approach 15

3.1 Silica as Filler - Properties and State of the Art . . . . . . 16

3.2 Copolymerization of Styrene and Butadiene . . . . . . . 16

3.3 Functionalization of Silica Nanoparticles . . . . . . . . . . . 20

3.3 .1 Grafting-to Approach . . . . . . . . . . . . . . . . . 20

3.3.2 Grafting-from Approach . . . . . . . . . . . . . 23

3.4 Functionalized Silica Nanoparticles as Filler in Tire Compounds 26

3.4 .1 End Group Modification . . . . . . . . . . . . . . 26

3.4.2 Mixing of the Compounds and Production of the Test Specimens ................. 28

3.4 .3 Results of Physical Testing . . . . . . . . . . . . . . . 29

3.5 Conclusion and Future Perspectives $\ldots \ldots \ldots \ldots$ 


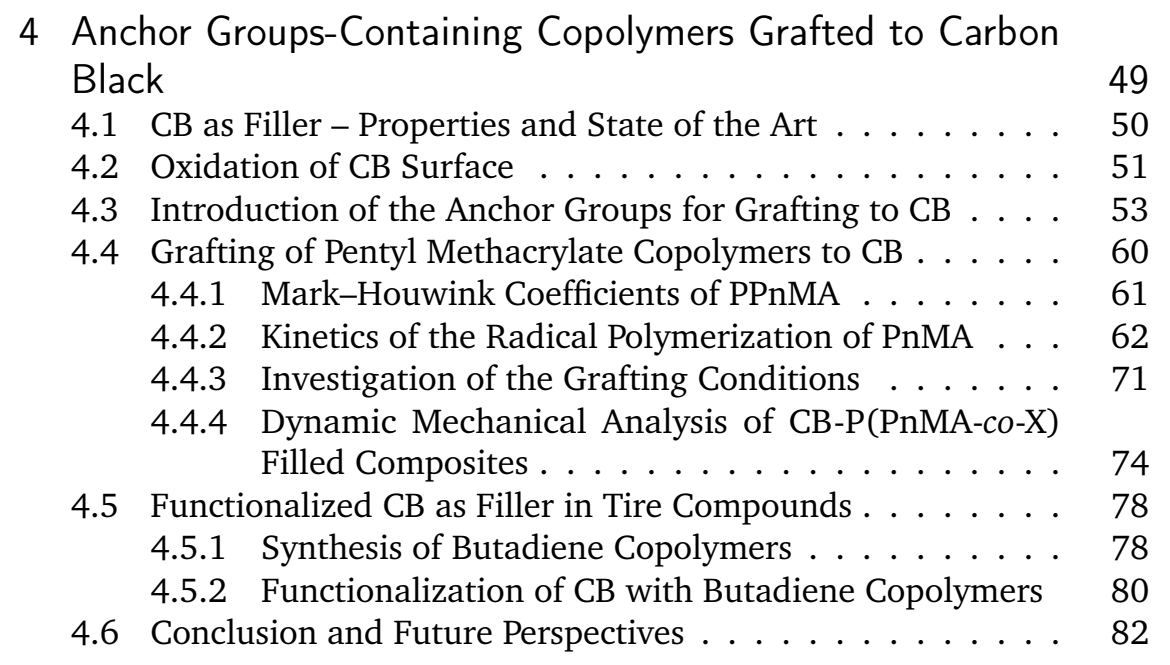

5 Closing Remarks 87

6 Experimental Section 91

6.1 Chemicals . . . . . . . . . . . . . . . . . . . . . . . 91

6.2 Synthesis of Monomers . . . . . . . . . . . . . . . . 91

6.2.1 Glycidyl 4-vinylbenzyl ether (GMSt) . . . . . . . . . 91

6.2.2 4-Furfuryloxymethylstyrene (FMSt) . . . . . . . . . 92

6.3 Synthesis of RAFT Agents $\ldots \ldots \ldots \ldots$. . . . . . . . . 92

6.3 .1 Benzylpropyltrithiocarbonate (BPTT) . . . . . . . . 9 92

6.3.2 Activated 4-Cyano-4-[(dodecylsulfanylthiocarbonyl) sulfanyl]pentanoic acid (Act. CDSPA) $\ldots \ldots . .969$

6.3.3 Dodecyl-((trimethoxysilyl)ethylphenylmethyl) trithiocarbonate (DTPT) . . . . . . . . . . . . 9 93

6.3.4 Propyl-((trimethoxysilyl)ethylphenylmethyl) trithiocarbonate (PTPT) $\ldots \ldots \ldots \ldots \ldots$. . . . . . . . 94

6.4 RAFT Polymerizations $\ldots \ldots \ldots \ldots$

$6.4 .1 \quad$ Butadiene Copolymers . . . . . . . . . . . . . . . . 94

6.4.2 Methacrylate Homo- and Copolymers . . . . . . . . . 95

6.5 Synthesis and Functionalization of Silica Nanoparticles . . . 95

6.5 .1 Synthesis of Silica Nanoparticles via Stöber Method. 95

6.5.2 Immobilization of RAFT Agent with Anchor Group . 96

6.5.3 Immobilization of RAFT Agent without Anchor Group 96 
6.5.4 Grafting of Polymer to Silica Nanoparticles via ThiolEne Click Reaction . . . . . . . . . . . 96

6.5.5 Grafting of Polymer to Silica Nanoparticles via Nucleophilic Substitution. . . . . . . . . . . 97

6.5 .6 Thioether End Group . . . . . . . . . . . . . . . . . . . 97

6.6 Functionalization of Carbon Black . . . . . . . . . . . . 97

6.6 .1 Synthesis of CB-COOH . . . . . . . . . . . . 97

6.6 .2 Synthesis of $\mathrm{CB}-\mathrm{OH} \ldots \ldots$. . . . . . . . . . 98

6.6 .3 Grafting of Polymer to CB . . . . . . . . . . . . 98

6.7 Instrumentation . . . . . . . . . . . . . . 98

6.7 .1 Density Measurement . . . . . . . . . . . . . . . 9 98

6.7.2 Dynamic Mechanical Analysis . . . . . . . . . . . . . 98

6.7 .3 Elemental Analysis . . . . . . . . . . . . . . . . 99

6.7.4 Nuclear Magnetic Resonance Spectroscopy. . . . . . 99

6.7 .5 PLP-SEC . . . . . . . . . . . . . . . . 99

6.7.6 Size-Exclusion Chromatography . . . . . . . . . . 100

6.7 .7 Thermogravimetric Analysis . . . . . . . . . . . . 100

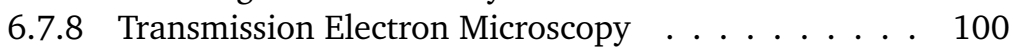

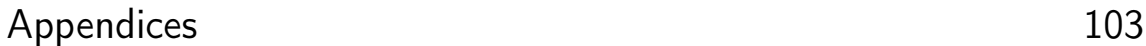

\begin{tabular}{ll}
\hline Abbreviations & 117
\end{tabular}

\begin{tabular}{ll}
\hline Bibliography & 121
\end{tabular} 



\section{Introduction and Motivation}

The use of inorganic materials, like silica (Si) and carbon black (CB), as fillers in plastics is of huge industrial interest. ${ }^{[1,2]}$ One main field of application is their usage as reinforcing filler in rubber compounds. Through polymerfiller interactions the stiffness and the elastic modulus of the compound are enhanced. The rubber becomes more resistant and less prone to crack formation. [3,4]

The combination of organic and inorganic materials is challenging, since filler-filler interactions are strong, leading to aggregation of the nanoparticles within the polymeric matrix. In contrast, the polymer-filler interactions are mostly weak. [5] Thus, improvement of the polymer-filler interactions is of huge interest to the industry and the functionalization of inorganic nanoparticles is a continuously growing field of research. ${ }^{[6]}$ An important quantity in this context is the dispersibility of the nanoparticles within the polymeric matrix. The more finely distributed the particles are, the more particle surface is present to interact with the polymer. [7]

There is already a variety of approaches known to enhance particle dispersion either chemically or physically. Wu et al. excited CB particles using a high-energy electron beam, resulting in a decrease of the particle size and an increase of the ratio of oxygen-containing functional groups at the surface. It was shown, that within a matrix of natural rubber the polymer-filler interactions were enhanced. ${ }^{[8]}$ Another approach is the use of so-called filler activators. These are small molecules that bind to the particle surface via adsorption or chemically, leading to an improvement of the polymer-filler interactions. [4,5] Typical filler activators for silica nanoparticles are organic silane compounds, which are the subject of extensive research and many patents. ${ }^{[5,9,10]}$ 
Besides small molecules polymer can also be covalently bound to inorganic nanoparticles. Springer showed, that the polymerization from CB surface via reversible addition-fragmentation chain transfer (RAFT) polymerization is possible. For this, particular RAFT agents were synthesized and bound to the surface via a Diels-Alder reaction. ${ }^{[11]}$ The polymerization from the silica surface has already been studied intensively using different polymerization techniques. Depending on the method, either controlling agents, monomer molecules or initiator molecules are bound to the surface prior to polymerization. Mostly silyl groups are used as surface anchor groups. [11-13]

This work focuses on the functionalization of silica and CB nanoparticles with (co)polymers to be used as reinforcing fillers in tire compounds. Modern tires are high performance materials, that consist of about $30 \%$ of fillers. ${ }^{[14]}$ Tires have a huge impact on the driving characteristics, like fuel consumption, driving comfort and safety. At the center of tire development is the so-called magic triangle of rolling resistance, wet braking and durability. Since optimizing one property usually leads to loss in another, there is a continuous development of tire materials in science and industry. ${ }^{[10,14]}$ In this context, the use of polymer-functionalized silica and CB nanoparticles as fillers could offer new possibilities to adjust the material properties. Besides, the comparison of the physical properties of compounds filled with functionalized and unfunctionalized particles as well as of typical tire compounds could give new insights to the polymer-filler and filler-filler interactions within cured compounds.

As for the synthesis of functionalized silica and CB particles different strategies for the grafting of polymer to the particle surface via RAFT polymerization are used and investigated with respect to the grafting mechanisms and resulting grafting densities. Silica nanoparticles are functionalized using the grafting-from approach with three different RAFT agents and the graftingto approach with two different grafting mechanisms. Another grafting-to approach using copolymers bearing anchor groups for the particle surface within the polymer side chain is adopted for the functionalization of CB particles. Thereby, three anchor groups are used and the grafting conditions, like reaction temperature and duration, are investigated. 


\section{Theoretical Background}

In this chapter the theoretical background of the functionalization of inorganic nanoparticles will be summerized briefly. This includes the applied technique of RAFT polymerization as well as some aspects of radical copolymerizations. Furthermore the impact of inorganic nanoparticles in rubber compounds will be presented. Additional concepts and background will be explained on the point it is required within this work.

\subsection{Fundamentals of RAFT Polymerization}

Within the last decades, polymeric materials became essential elements of everyday life. Many fields of application have been developed, like the use of smart polymers, responsive to temperature or $\mathrm{pH}$, in medicine or the application of conductive polymers in optoelectronic devices. [15-17] This leads to a continually increasing demand on high-performance materials with well-defined and controllable properties: starting from the applicability of a range of monomers under various reaction conditions up to the realization of diverse topologies and the design of hybrid materials. [18-20] To meet these challenges, polymerization techniques are continuously developed further. Starting in the 1990's the reversible-deactivation radical polymerization (RDRP) mechanism was introduced. ${ }^{[21-23]}$ Since then, techniques like the RAFT polymerization have become established in polymeric research. [18,19,21]

The RAFT polymerization was introduced by Rizzardo et al. in 1998. [21] By the use of a dithioester chain transfer agent (Figure 2-1) polymers with narrow molecular weight distributions (MWD) can be obtained. In contrast to other radical polymerization techniques, the RAFT mechanism is tolerant 
<smiles>[R]SC([Z])=S</smiles>

Figure 2-1: General structure of a RAFT agent. R displays the leaving group and $Z$ the stabilizing group.

towards many functional groups and solvents. Furthermore, it provides the opportunity of copolymerizations and the synthesis of hybrid materials. [24-27]

\subsubsection{The RAFT Mechanism}

The mechanism of RAFT polymerization is analogue to that of a conventional radical polymerization with two additional steps, based on additionfragmentation equilibrium (Scheme 2-1), ${ }^{[28,29]}$ The reaction is started by the decomposition of an initiator $\left(\mathbf{l}^{\bullet}\right)$, commonly azo or peroxide compounds, and subsequent addition of monomer molecules (M) forming a propagating radical $\left(\mathrm{P}_{\mathrm{n}}^{\bullet}\right)$. Through addition of this radical to the CTA in the pre-equilibrium an intermediate (1) is formed. Fragmentation of the leaving group $\left(R^{\bullet}\right)$ leads to initiation of further monomer molecules, forming a new propagating radical $\left(\mathrm{P}_{\mathrm{m}}^{\bullet}\right)$. In the main equilibrium a rapid exchange of the active propagating radicals $\left(\mathrm{P}_{\mathrm{n}}^{\bullet}\right.$ and $\mathrm{P}_{\mathrm{m}}^{\bullet}$ ) and the dormant polymeric thiocarbonylthio compound (2) sets in, leading to a simultaneous growth of all polymer chains.

In contrast to other RDRP techniques, the termination of the propagating radicals is not suppressed. Nevertheless after stopping the polymerization most chains posses a thiocabonylthio end group that can be used either for further functionalized or as macroRAFT agent in following polymerizations. ${ }^{[25] 30]}$ The choice of the RAFT agent depends on the monomer being polymerized. While the $Z$ group activates the addition of radicals to the $\mathrm{C}=\mathrm{S}$-bond and stabilizes the RAFT intermediates ( 1 and 3 ), the $\mathrm{R}$ group needs to be a good leaving group in contrast to the propagating polymer chain and has to be able to initiate the monomer molecules. [19,31,32]

\subsubsection{RAFT Polymers on Surfaces}

The surface functionalization via RAFT polymerization can be divided into three approaches: grafting-from, grafting-to and grafting-through. Depending on the synthetic route, one component of the polymer is grafted before or 


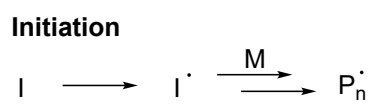

Pre-equilibrium

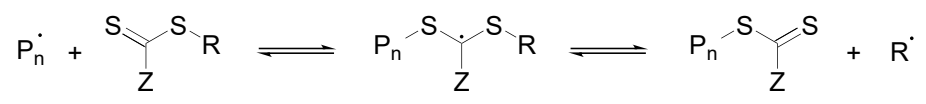

\section{Reinitiation}<smiles></smiles>

\section{Main equilibrium/propagation}

$$
P_{n}^{\cdot}+{ }_{Z}^{S_{Y}}{ }^{S_{P_{m}}} \rightleftharpoons P_{n}{ }^{-S_{Y}} Y_{Z}^{-S_{P_{m}}} \rightleftharpoons P_{n}{ }^{-S_{Y}} Y_{Z}^{S}+P_{m}^{\cdot}
$$

(2)
(2)

Termination

$$
\mathrm{P}_{\mathrm{n}}^{\cdot}+\mathrm{P}_{\mathrm{m}}^{\cdot} \longrightarrow \mathrm{P}_{\mathrm{m}+\mathrm{n}} \quad \mathrm{P}_{\mathrm{n}}^{\cdot}+\mathrm{P}_{\mathrm{m}}^{\cdot} \longrightarrow \mathrm{P}_{\mathrm{n}}+\mathrm{P}_{\mathrm{m}}
$$

Scheme 2-1: Mechanism of RAFT polymerization. [29]

after polymerization to the surface. ${ }^{[33]}$ This can be done either by physisorption or chemically through an anchor group. The choice of this anchor group depends an the surface being functionalized. 34,35$]$

In the grafting-through approach monomer molecules are bound to the surface (Scheme 2-2) and are incorporated into the polymer chain during the polymerization. ${ }^{[36]}$ Since the incorporation is random, the properties of the surface bound polymer can be hardly controlled and by addition of more than one surface bound molecule the formation of loop chains is possible. 37 ]

The binding of pre-synthesized polymer chains to the surface is called grafting-to approach (Scheme 2-2). The anchor group can be located either within the polymer side chain or at its end group. ${ }^{[12] 38]}$ Since the polymer is synthesized prior to grafting, its properties can be controlled well and it can be analyzed easily. ${ }^{[37]}$ However, through steric hindrance of the polymer chains, only low grafting densities can be achieved. [33]

In the grafting-from approach the polymer growth at the surface. In case 

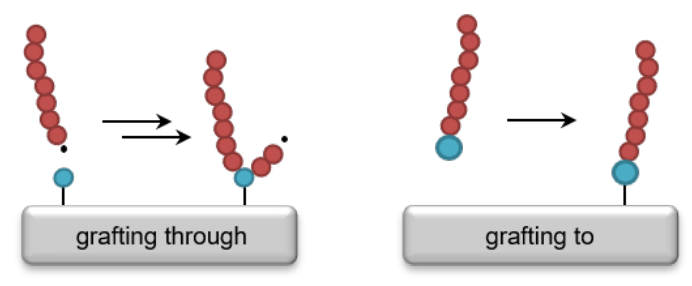

Scheme 2-2: Schematic representation of grafting-through and graftingto approaches.
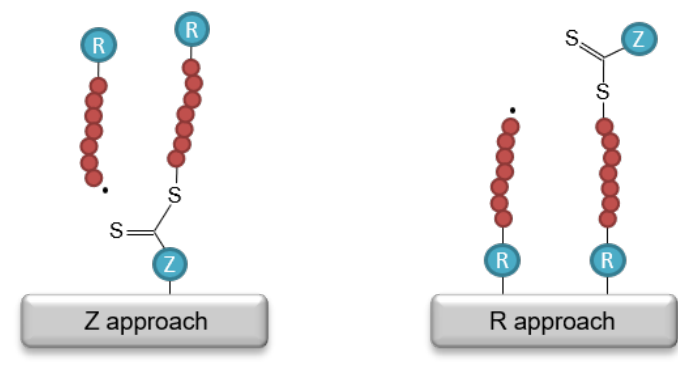

Scheme 2-3: Schematic representation of R and Z group approach of grafting-from approach via RAFT polymerization.

of RAFT polymerization the RAFT agent is anchored to the surface either via its $R$ or $Z$ group (Scheme 2-3). ${ }^{[12,34,37]}$ The binding of the $Z$ group leads to chain growth in solution, since the RAFT unit remains at the surface all time. Advantageous with this approach is that only living chains are grafted to the surface, which can be cleaved easily for analysis. ${ }^{[27,39-41]}$ With increasing length of the growing chains the accessibility of the surface bound RAFT group decreases, resulting in low grafting densities as with the grafting-to approach. ${ }^{[42]}$ In case of the $R$ approach the chains grow from the surface and the RAFT unit is located at the end of the chain. As a result, high grafting densities can be achieved and subsequent end group functionalization is possible. However, termination or formation of polymer networks may occur. ${ }^{[34,43,44]}$ For analysis of the polymer either the anchor group needs to be cleaved off or the surface needs to be dissolved chemically. [12,45] 
2.2 Fundamentals of Radical Copolymerizations

\begin{tabular}{|c|c|}
\hline ЗАВАВАВАВАВАВАВАВ & Alternating \\
\hline АВBAАВАВBABABABBAB & Statistical \\
\hline AAABBAAABABABBAB & Gradient \\
\hline AAAAAAAABBBBBBBB & Block \\
\hline
\end{tabular}

Figure 2-2: Overview of the copolymer classes consisting of two kinds of monomers.

\subsection{Fundamentals of Radical Copolymerizations}

Copolymers consist of more than one kind of monomer and are classified according to the sequence within the polymeric chain. ${ }^{[46]}$ Figure $2-2$ shows the classes of copolymers consisting of two types of monomers. In case of alternating, statistical and gradient copolymers the sequence is determined by the kinetic parameters of the radical copolymerization. ${ }^{[47,48]}$ The monomer radical at the end of the propagating chain can either add a monomer of the same class or one of the other. This results in four possible propagating steps, characterized by the corresponding rate coefficients of homo $\left(k_{11}\right.$ and $\left.k_{22}\right)$ and cross $\left(k_{12}\right.$ and $\left.k_{21}\right)$ propagation. The reactivity ratios $\left(r_{1}\right.$ and $r_{2}$, Equation (2.1p), also referred to as copolymerization parameters, give information about the mechanism of copolymerization and are tabulated for a variety of monomer combinations: ${ }^{[48,49]}$

$$
r_{1}=\frac{k_{11}}{k_{12}} \quad r_{2}=\frac{k_{22}}{k_{21}} .
$$

If $r>1$ the monomer radical favors the addition of the same type, $r<1$ means the preference of adding the other monomer. In case of $r=1$ the addition of both kinds of monomers is equally probable. Using the copolymerization equation of Mayo and Lewis, the composition of the copolymer can be calculated from the ratio of monomers within the initial mixture and the corresponding reactivity ratios. ${ }^{[48,49]}$

A prominent way to synthesize block copolymers is the use of RAFT polymerization. ${ }^{[50]}$ The first block is polymerized and afterwards used as macroRAFT agent in a second polymerization. The sequence is determined by the monomer combination, since the polymeric chain in the macroRAFT agent needs to be a suitable R group for the second monomer (see Subsection 2.1.1). ${ }^{[50]}$ While in statistical copolymers the monomer properties 
(hydrophilic-hydrophobic, plastic-elastic) are mixed, block copolymers develop micro phases with associated properties. ${ }^{[50,51]}$

\subsection{Fundamentals of Tire Materials}

In 1845, shortly after the invention of vulcanization by Goodyear, the first solid rubber tire for horse-drawn carriage was brought to market. [52,53] Since then, continuous development of tire construction and materials is an important field in science and industry. $[10,14,53,54]$

\subsubsection{Composition of Tire Materials}

The construction of a modern tire is complex, consisting of several segments made of rubber compounds and reinforcing supports, like steel or textile fibers. ${ }^{[14,55]}$ A typical composition of a rubber compound is given in Table 2 1. ${ }^{[14]}$ Depending on the field of application, natural rubber, mainly used in truck tires, or synthetic rubbers, like styrene butadiene rubber (SBR), are used. Fillers like silica and $\mathrm{CB}$ reinforce the rubber, resulting in the reduction of abrasion and rolling resistance. The viscosity and the hardness of the compound can be tuned by addition of oil or resin. Antioxidants protect the tire from crack formation, due to oxidation by UV light. Besides, sulfur, zinc oxide and stearic acid as activators and organic sulfur compounds as accelerators, are needed for vulcanization. ${ }^{[53,54]}$

Modern tires are complex technical products with a high impact on the driving characteristics like comfort, fuel consumption and safety. Their

Table 2-1: Typical composition of rubber compound for tire in parts per hundred rubber (phr). [14]

\begin{tabular}{lr}
\hline & Amount / phr \\
\hline Rubber (natural or synthetic) & 100 \\
Filler (silica, CB,...) & 50 \\
Softner (resin, oil) & 10 \\
Chemicals for vulcanization (sulfur, zinc & 7 \\
oxid, stearic acid, activators) & \\
Antioxidants & 5 \\
\hline
\end{tabular}


properties depend on the field of application and on the environmental conditions. As summer tires become hard in winter, resulting in bad wet braking performance, winter tires get soft in summer, leading to enhanced rolling resistance and fuel consumption. [14]

Tire development is based on the so-called magic triangle of rolling resistance, wet braking traction and abrasion resistance. Rolling resistance affects e.g. fuel consumption, wet braking resistance is important for the safety and high abrasion resistance decreases the environmental impact. Since the improvement of one property leads to degradation of another, the continuous improvement of tire materials is of great importance. [10,14,55]

\subsubsection{Mechanical Properties of Rubber Compounds}

In the focus of this research is the improvement of polymer-polymer, polymerfiller and filler-filler interactions, which effect the mechanical properties of

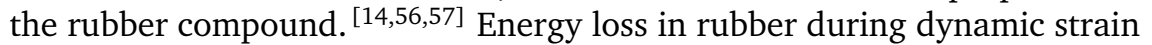
effects rolling resistance, fraction and skid resistance. The repeated straining of the tire due to rotation and braking is a process of constant energy input involving different temperatures and frequencies as can be seen in Table 2-2. [57] The time-temperature equivalence principle (WLF-temperaturefrequency conversion) allows the conversion of temperature to frequency to make high frequency data available, thereby high frequencies correspond to low temperatures. [58,59]

If a stress $(\sigma)$ is applied periodically with a sinusoidal alternation to an elastomer with viscoelastic behaviour, the answer of the material will be a strain $(\varepsilon)$, which shows also sinusoidal alternation but is out of phase with the stress (Figure 2-3). This phase shift is referred to as $\delta$. The dynamic stress-strain behaviour of an elastomer material can be described with the storage modulus $\left(E^{\prime}\right)$, which is in phase with stress, and the loss modulus

Table 2-2: Corresponding temperatures and frequencies $(\nu)$ of processes during tire performance. [57]

\begin{tabular}{lcc}
\hline & $\nu / \mathrm{Hz}$ & $T /{ }^{\circ} \mathrm{C}$ \\
\hline Rolling resistance & $10-100$ & $50-80$ \\
Wet grip & $10^{4}-10^{7}$ & 20 \\
\hline
\end{tabular}




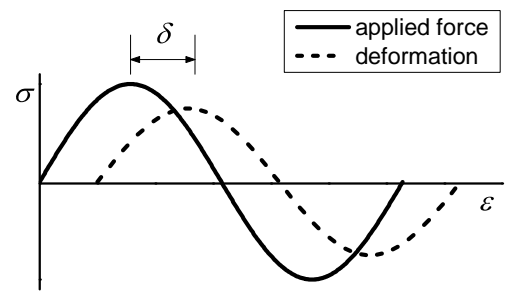

Figure 2-3: Sinusoidal stress strain correspondence.

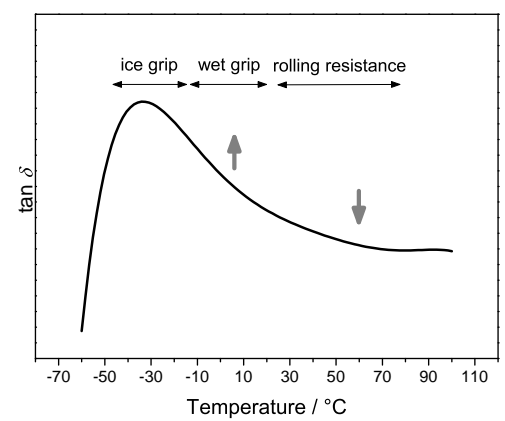

Figure 2-4: Typical $\tan \delta$ curve of a tire compound. [57]

$\left(E^{\prime \prime}\right)$, which is $90^{\circ}$ out of phase. The storage modulus is proportional to the hardness of a material, for rubbers the moduli are typically given in MPa. The loss factor $(\tan \delta$, Equation (2.2)) is the ratio of loss and storage modulus: [57]

$$
\tan \delta=\frac{E^{\prime \prime}}{E^{\prime}} .
$$

As rolling resistance should be minimized in order to safe energy, a low $\tan \delta$ is favored around 50 to $80^{\circ} \mathrm{C}$, high hysteresis around -20 to $0^{\circ} \mathrm{C}$ is necessary for good wet grip and skid resistance (Figure 2-4). ${ }^{[54,57]}$ 


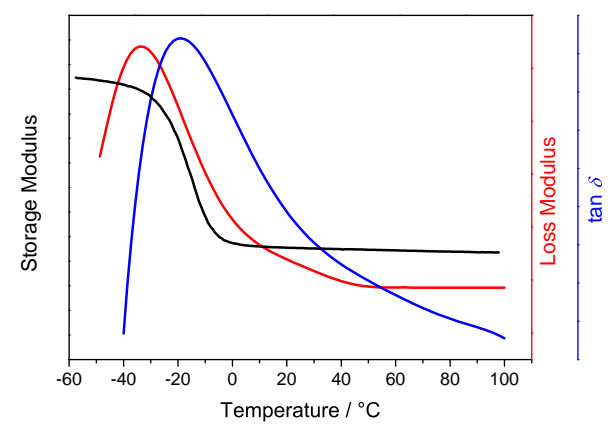

Figure 2-5: Exemplary illustration of temperature-dependent $\tan \delta$, storage and loss modulus of rubber compound. [57]

\section{Unfilled Rubber}

Below the glass transition temperature $\left(T_{\mathrm{g}}\right)$ the chain movement is limited, resulting in a high viscosity and a small free volume. In this glassy state the storage modulus is high, $\tan \delta$ and $E^{\prime \prime}$ are low (Figure 2-5). The segment motion and the free volume of the polymer increase with temperature, resulting in rapid decrease of the viscosity around $T_{\mathrm{g}}$ and high hysteresis. In the transition zone between the $T_{\mathrm{g}}$ and the rubber state, the loss modulus and the loss factor go through maxima and $E^{\prime}$ drops by several orders of magnitude. In the rubber state the thermal energy is comparable to potential energy barriers of segment motion, leading to high entropic elasticity, low resistance to strain and low energy dissipation during dynamic deformation. [57]

\section{Filled Rubber}

The dynamic properties of filled elastomers depend on the polymer-filler and filler-filler interactions, mainly the dispersion of the particles within the rubber matrix and the amount of rubber bound onto the filler surface. [54,57] This so-called bound rubber is a layer of immobilized polymer through physical adsorption or chemisorption at the surface with limited segment motion. Therefore it is in a quasi-glass state with higher storage modulus than the free matrix polymer. $[57,60,61]$

If the dispersion of the filler particles within the rubber matrix is not 
sufficient, the filler network may not be fully formed or the particles might aggregate. In these aggregates or agglomerates some rubber is trapped and cannot participate during the deformation. Both bound rubber and bad dispersion lead to an increase of the effective volume fraction of the filler and of the viscosity of the vulcanizate. $[54,57]$

\section{Temperature-Dependence of Dynamic Properties of Filled Rubber}

For rubber compounds filled with carbon black $E^{\prime}$ and $E^{\prime \prime}$ increase with filler loading over the range of temperature, thereby the increase is little at low temperatures and large at high temperatures. The situation is different for $\tan \delta$, which can be classified by temperature. At low temperatures $\tan \delta$ decreases with filler loading, at high temperature it increases with filler loading, but the temperature at which the maximum occurs does not change. [57,62]

The hysteresis at high temperatures of filled elastomers originates from additional energy dissipation through bound rubber and formation and breakdown of the filler network. With increasing temperature the hysteresis decreases, because less rubber is bound to the particles. At temperatures between the glassy state and the $T_{\mathrm{g}}$ some rubber is trapped within the filler network, which cannot be broken down at these temperatures. This leads to a reduction of the effective polymer volume, resulting in a lower hysteresis. [57,62]

Both the amount of bound rubber and the formation of filler network are influenced by the size, structure and surface energy of the filler particles. The effect of particle morphology results in the same tendency that can be observed for increasing filler loading. Small particles have a higher specific surface area and can therefore bind more rubber. Furthermore, the inter particle distance decreases, leading to a stronger filler network. Both cause a larger hysteresis in the rubber state. $[57,62,63]$

In contrast to carbon black, the polymer-filler interactions in silica filled vulcanizates with hydrocarbon polymers are only weak, due to the polar surface of the particles. Here the formation of bound rubber is unlikely and the hysteresis behaviour of the vulcanizate is based on the filler-filler interactions. ${ }^{[57,62]}$ At temperatures below the $T_{\mathrm{g}}$ the hysteresis is larger for silica filled elastomers, because of a higher effective polymer volume in the vulcanizate. In the transition zone the hysteresis is higher for carbon black, as a result from continuous breakdown and reformation of the filler network. In the rubber state silica filled vulcanizates show an increase 
in hysteresis at high temperatures, due to weakening of the filler-filler interactions and an increasing portion of filler network, that can be broken down and reformed. [57,62,64]

\section{Strain Dependence of Dynamic Properties of Filled Rubber}

The storage modulus of a filled compound decreases non-linear with strain. This behaviour is known as the 'Payne Effect' and is related to the filler network formed in the polymer matrix. ${ }^{[57,65]}$ The storage modulus can be divided into four aspects, illustrated in Figure 2-6. The polymer-polymer part (I) describes the energy needed to stretch the polymer network without filler. The addition of filler particles reduces the mobility of the polymer during strain. This hydrodynamic effect (II) results in an increase of energy demand for deformation. As the polymer-filler interactions (III) are enhanced, e.g. by physisorption, the mobility of the polymer is decreased further and the energy demand increases. [14,64]

As the already mentioned parts are independent of strain, the filler-filler interactions (IV) lead to a decrease of storage modulus with strain. At low strain the filler network cannot be broken and the modulus is determined by the hydrodynamic effect of the filler. At high strain the rate of breakdown is higher than the rate of reformation of the filler network, leading to a low modulus at high strain. The Payne effect is reduced with enhanced dispersion of the filler within the matrix. $[14,54,57,66]$

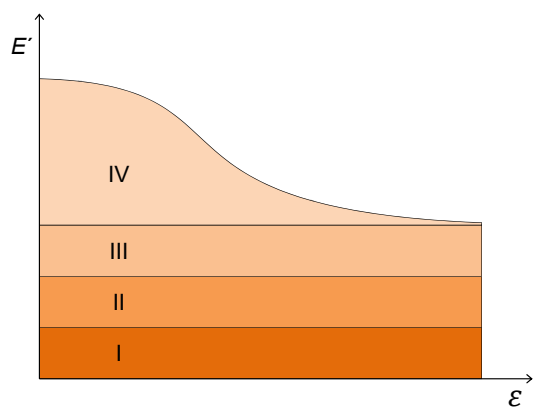

Figure 2-6: Schematic illustration of the strain dependent storage modulus for filled vulcanizate according to Payne. [65] 



\section{SBR-Functionalized Silica}

\section{Nanoparticles - Grafting-from versus Grafting-to Approach}

In this chapter, the influence of the functionalization of silica nanoparticles on their properties as filler in typical tire compounds is investigated. At first, the polymerization of SBR via RAFT polymerization in a high-pressure autoclave is introduced. Subsequently, the synthesis and functionalization of silica nanoparticles is presented. The grafting-to approach was conducted using two different reaction mechanisms for binding the polymer to the particles. Polymerizations via the grafting-from approach were performed with three different RAFT agents. Finally, the processing of rubber compounds, containing functionalized silica nanoparticles, at the mixing lab of Continental is presented and the results of physical tests are discussed. The results presented in this chapter are part of an invention disclosure, which has already been submitted and the patent is under preparation. [67]

The denotation of the samples used within this chapter consists of two parts, referring to the synthetic route of functionalization. The first part stands for the approach used, either grafting-to (GT) or grafting-from (GF). In case of GT-samples, the second part refers to the chemical reaction used for functionalization. For GF-samples the second part stands for the RAFT agent bound to the particles prior to polymerization. The corresponding unfunctionalized silica nanoparticles are labeled Si as second part. 
3 SBR-Functionalized Silica Nanoparticles - Grafting-from versus Grafting-to Approach

$$
(\mathrm{EtO})_{3} \mathrm{Si} \frown \mathrm{S}^{-} \mathrm{S} \sim \mathrm{Si}(\mathrm{OEt})_{3}
$$

Figure 3-1: TESPD as a typical example of a filler activator for silica nanoparticles used in rubber industry.

\subsection{Silica as Filler - Properties and State of the Art}

Silica nanoparticles consist of a network of silicon dioxide units with hydroxy groups at the surface, which mainly determine the chemical and physical properties. ${ }^{[68,69]}$ Primary particles are spherical, amorph and very hydrophilic. Through hydrogen bonds, nanometric aggregates are formed, that build up higher agglomerates. ${ }^{[69]}$ Properties like shape, size and surface reactivity are determined by the manufacturing process. According to this, they are classified as fumed, Stöber or precipitated silica. [3]69,70]

Silica nanoparticles can be found in many everyday products and since the 1940's, they are used as reinforcing fillers in tire compounds, partly replacing CB. ${ }^{[2,10,14,71]}$ The functionalization of silica nanoparticles is of huge interested in science for application in various fields, like medicine or rubber industry, in order to further improve products. ${ }^{[15,33,72]}$ Many approaches for the grafting of small molecules or polymers are already known. Most of them involve silyl units as anchor groups for the silica surface. $12,44,73]$

Due to the hydrophilic nature of the silica surface, polymer-filler interactions within hydrocarbon polymer compounds are weak. Therefore, in rubber industry, silica nanoparticles are silanized either prior to mixing or in situ with filler activators. [57,71] These are bifunctional chemical compounds, that enable interactions between filler particles and matrix polymer. Mostly organic silane compounds containing sulfur functional groups, like bis(triethoxysilylpropyl) disulfide (TESPD, Figure 3-1), are used, leading to crosslinking during vulcanization. [65!68,71]

\subsection{Copolymerization of Styrene and Butadiene}

Styrene butadiene copolymers are widely used synthetic rubbers. ${ }^{[10,74]}$ Especially the statistic copolymer SBR, which is industrially synthesized via emulsion or solution polymerization, is of great importance. [74,75]

The properties of SBR depend on the micro- and macrostructure of the copolymer. The microstructure is defined by distribution and configuration 


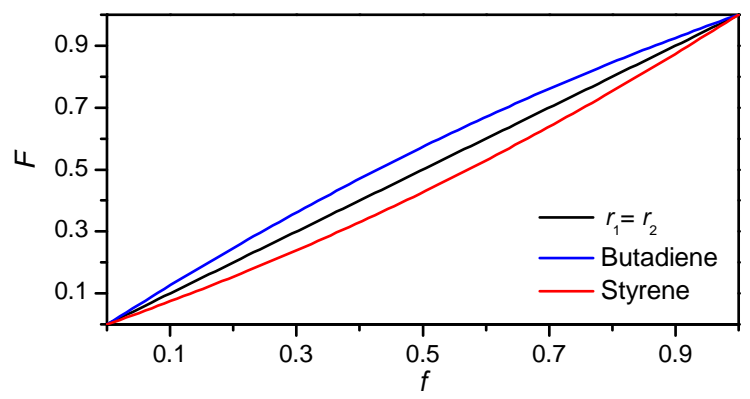

Figure 3-2: Polymer composition as function of monomer composition of radical polymerization of styrene $(r=0.78)$ and butadiene $(r=$ 1.39). [49]

of monomers within the polymer chain. ${ }^{[3]}$ The synthesis of SBR via radical copolymerization leads to a statistical copolymer, so the properties of thermoplastic styrene $(\mathrm{St})$ and elastic butadiene $(\mathrm{Bd})^{\mathrm{i}}$ are mixed. Figure $3-2$ shows the copolymerization diagram of SBR. Typically a ratio of styrene about $25 \mathrm{~mol} \%$ is used, therefore SBR can be classified as an elastomer. [3,74]

The configuration of butadiene within the polymer chain is determined by the remaining double bond. In case of 1,2-butadiene, it is located in the side chain. In 1,4-butadiene it is located in the polymer backbone either in trans or cis configuration. ${ }^{[3,74,75]}$ The polymer composition and configuration of butadiene can be determined using ${ }^{1} \mathrm{H}$-nuclear magnetic resonance (NMR) spectroscopy (Figure 3-3). ${ }^{[76]}$ The macrostructure is determined by the chain length, influencing the stiffness of the polymer, and possible crosslinking of the conjugated diene, leading to a high dispersity. ${ }^{[3,20]}$ In radical polymerizations the degree of crosslinking increases with conversion, monomer concentration and temperature. If high molecular masses are targeted, this leads to a conflict, since the propagation rate coefficient of butadiene in bulk and solution is extremely low. ${ }^{[20,77]}$ Additionally, retardation might occur in case of RAFT polymerization, resulting in extension of the reaction period. [20]

In contrast to other monomer classes, the polymerizations of butadiene

${ }^{\mathrm{i}}$ Butadiene consists of two constitutional isomers: 1,2-butadiene and 1,3-butadiene. The 1,2-isomer does not have any importance within this work therefore butadiene will be used synonymous for 1,3-butadiene. 


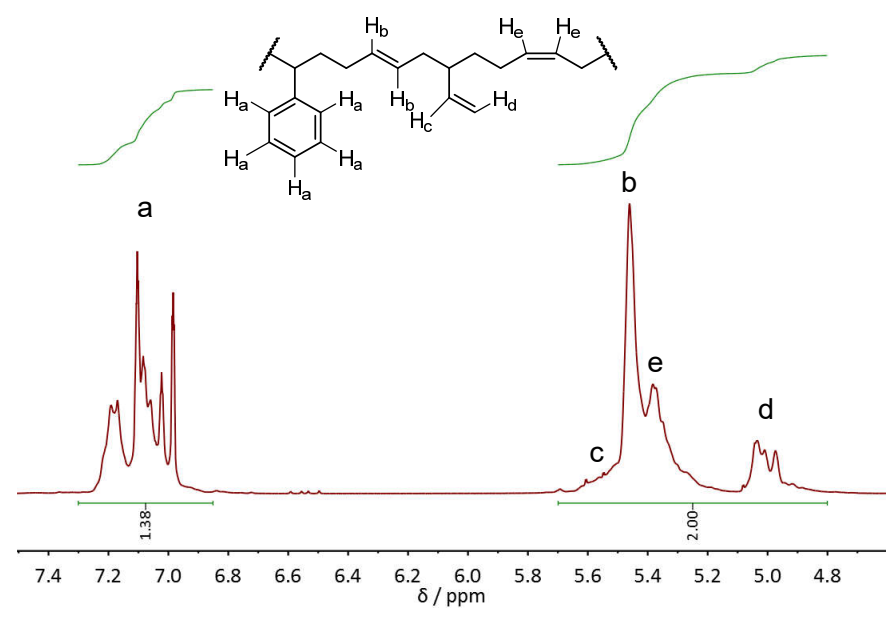

Figure 3-3: Exemplary presentation of ${ }^{1} \mathrm{H}-\mathrm{NMR}$ spectra of SBR and assignment of signals.

and other diene monomers have not been study intensively yet. This is mainly caused by the additional experimental expense, since most of these monomers are gaseous at room temperature (RT) and polymerization requires an appropriate setup. ${ }^{[20,77]}$ In this work, butadiene polymerizations were conducted using a high-pressure autoclave setup (Figure 3-4) designed and constructed by Springer. ${ }^{[11]}$ The reaction vessel $(450$ or $1900 \mathrm{~mL})$ is equipped with a stirrer and the temperature is controlled by a thermocouple and a thermostat. Liquid and solid components are added to the vessel and purged with nitrogen. The amount of used butadiene is controlled by condensation in an autoclave prior to the transfer into the reaction vessel. Upon completion of the polymerization, residual butadiene is burned and the remaining solution is removed from the vessel through an outlet at its bottom. 


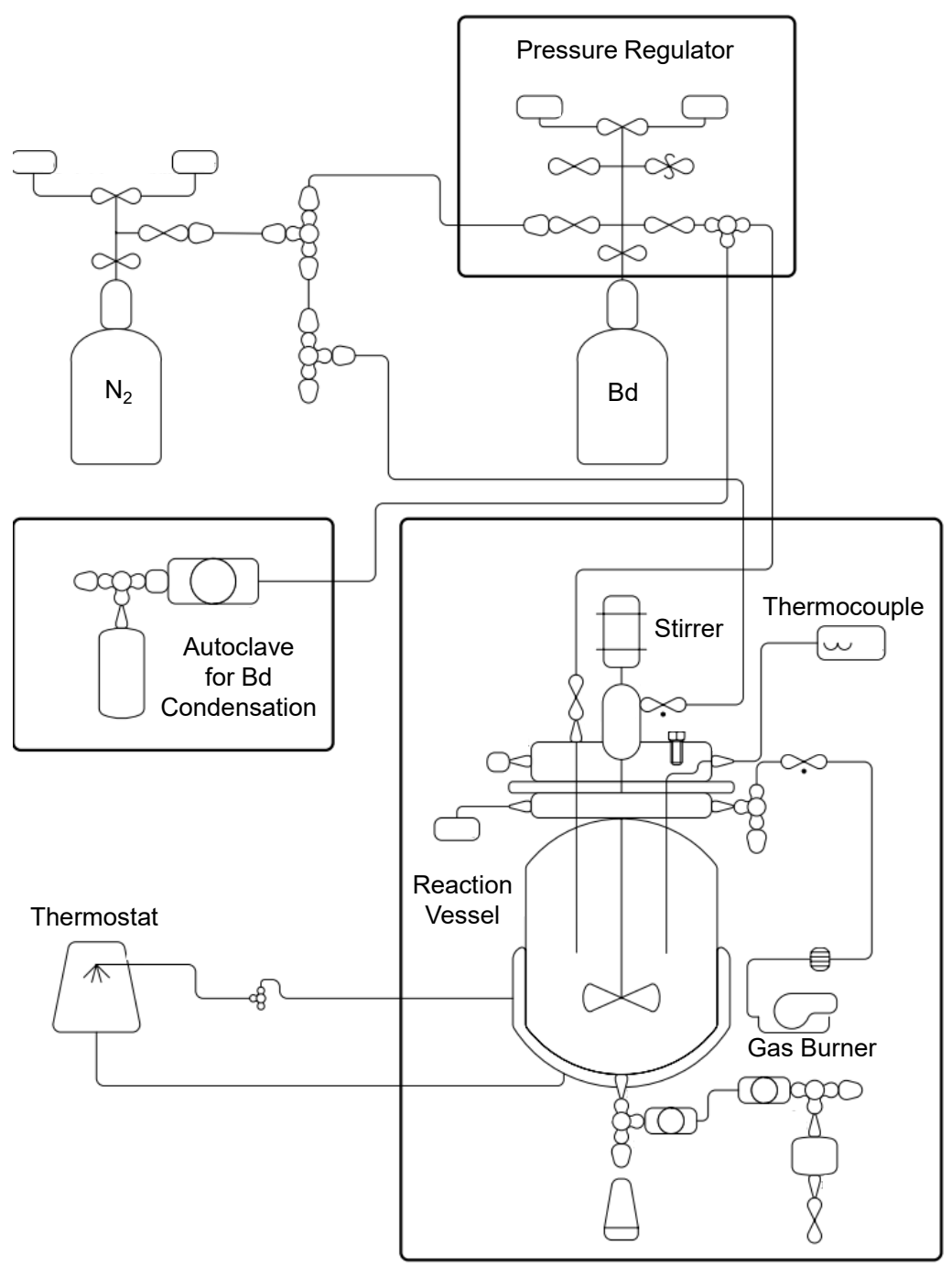

Figure 3-4: Setup of high-pressure autoclave for butadiene (Bd) polymerizations designed and constructed by Springer. [11] 
3 SBR-Functionalized Silica Nanoparticles - Grafting-from versus Grafting-to Approach

\subsection{Functionalization of Silica Nanoparticles}

The butadiene polymerization in presence of silica nanoparticles in the highpressure autoclave is challenging, in particular regarding the dispersibility of the particles during polymerization. Aggregation leads to crosslinking and formation of globules in the size of several millimeters. Since the particles within these globules are chemically crosslinked, dispersion during mixing of the compound is impossible and resulting specimen are useless for physical testing. [78]

The dispersion of silica particles during polymerization is influenced by several factors, like solvent and choice of the particles itself. The range of solvents is limited by used monomers and reaction conditions. Since butadiene is non-polar, most solvents suitable for the polymerization are bad solvents for the silica nanoparticles. ${ }^{[12]}$ As for the choice of silica particles, surface activity and shape are important. Particles should be well dispersable and should not tend to aggregate. Therefore particles prepared via Stöber method are more favorable than fumed silica. ${ }^{[78]}$

Two types of particles have been chosen for the different strategies for functionalization of silica nanoparticles in this work: Particles prepared via Stöber method (GT-Si) and particles purchased from PlasmaChem (GF-Si) in $50 \mathrm{wt} \%$ aqueous solution. Transmission electron micrographs of dried and redissolved particles in Figure A.1 show, that both types of particles are spherical and have only slight tendency to aggregate.

In the following, the different approaches used for functionalization of silica nanoparticles are presented and their advantages and disadvantages will be discussed.

\subsubsection{Grafting-to Approach}

One way to circumvent the problem of particle dispersion in the vessel is the use of a grafting-to approach for functionalization. However, unfavorable for this approach is, that typically low grafting densities are achieved (Subsection 2.1.2).

Grafting can be performed in a one or two step process, depending on the anchor group and reaction conditions. If the anchor group is already located at the polymer, it can be grafted directly to the surface in one step. In a two step process, a bifunctional coupling agent is grafted onto the surface prior to the functionalization. Grafting is performed via a chemical reaction 

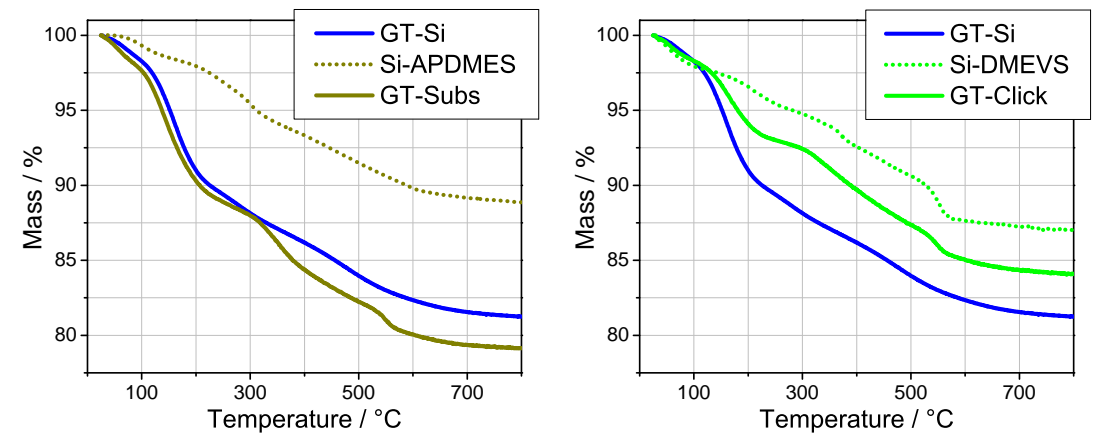

Figure 3-5: TGA curves of non-functionalized (GT-Si), aminofunctionalized (Si-APDMES), DMEVS-functionalized (Si-DMEVS), via nucleophilic substitution (GT-Subs) and thiol-ene click reaction (GTClick) functionalized silica nanoparticles measured under $\mathrm{N}_{2}$ atmosphere.

between a functional group of the polymer and the functional group of the coupling agent at the particle surface.

Hübner et al. showed, that crosslinking of silyl groups might occur during functionalization, leading to aggregation of the nanoparticles. To prevent this, monosilyl anchor groups need to be used. ${ }^{[12]}$ Due to the high reactivity of silyl groups with water, monosilyl anchor groups are not stable during polymerization and therefore grafting had to be conducted in a two step process.

Two reaction mechanisms have been chosen for the grafting-to approach: nucleophilic substitution and thiol-ene click reaction. Scheme 3-1 depicts the

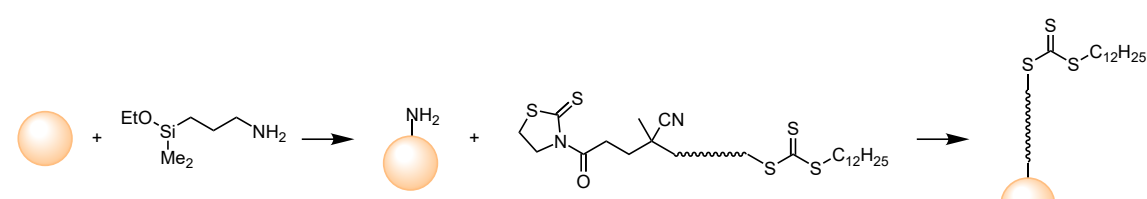

Scheme 3-1: Grafting-to approach for functionalization of silica nanoparticles via nucleophilic substitution (GT-Subs). APDMES was used as coupling agent and SBR was synthesized using activated CDSPA as RAFT agent. 
3 SBR-Functionalized Silica Nanoparticles - Grafting-from versus Grafting-to Approach

Table 3-1: Weight percentage of carbon, hydrogen and nitrogen measured by EA of non-functionalized (GT-Si), amino-functionalized (Si-APDMES), DMEVS-functionalized (Si-DMEVS), via nucleophilic substitution-functionalized (GT-Subs) and via thiol-ene click reactionfunctionalized (GT-Click) silica nanoparticles. Additionally, the number average molecular mass $\left(\bar{M}_{\mathrm{n}}\right)$ and the dispersity $(\nexists)$ of used polymers are given.

\begin{tabular}{lccccc}
\hline & $\bar{M}_{\mathrm{n}}$ & $\oplus$ & $\mathrm{C}$ & $\mathrm{H}$ & $\mathrm{N}$ \\
\cline { 4 - 6 } & $/ 10^{3} \mathrm{~g} \mathrm{~mol}^{-1}$ & & \multicolumn{3}{c}{$/ \mathrm{wt} \%$} \\
\hline GT-Si & & & 0.52 & 1.31 & 0.26 \\
Si-APDMES & & & 4.70 & 1.87 & 0.81 \\
GT-Subs & \multirow{2}{*}{17.6} & \multirow{2}{*}{1.7} & 6.22 & 2.03 & 1.66 \\
Si-DMEVS & & & 4.14 & 1.58 & 0.20 \\
GT-Click & 14.9 & 1.7 & 5.05 & 1.74 & 1.10 \\
\hline
\end{tabular}

reaction pathway of functionalization via nucleophilic substitution (GT-Subs). Silica nanoparticles are functionalized with 3-aminopropyldimethylethoxysilane (APDMES) to yield amino-functionalized silica nanoparticles (SiAPDMES) and SBR is synthesized with activated 4-cyano-4-[(dodecylsulfanylthiocarbonyl)sulfanyl]pentanoic acid (CDSPA) as RAFT agent. ${ }^{[79]}$ The results from thermogravimetric analysis (TGA) are shown in Figure 3-5. The twostep weight loss of GT-Subs between 300 and $600^{\circ} \mathrm{C}$ is characteristic for SBR (Figure B.1), indicating successful functionalization of the silica nanoparticles. Quantitative analysis is not possible from the TGA curves, since the pure silica particles also show a significant weight loss within this temperature region. This can be assigned to solvent molecules encapsulated during particle synthesis. The results from elemental analysis (EA) in Table 3-1 show an increase of carbon, hydrogen and nitrogen content for Si-APDMES compared to non-functionalized Si nanoparticles. After functionalization with SBR, the content of all three elements is further increased. Since the mass of SBR is mainly determined by the mass of carbon atoms, the mass fraction of polymer is approximately the difference of carbon content before and after functionalization with SBR, resulting in a mass fraction of $\sim 1.5 \mathrm{wt} \%$ of SBR on the silica particles and therefore a low grafting density as expected for a grafting-to approach. ${ }^{[33]}$

The reaction pathway of functionalization via thiol-ene click reaction is 


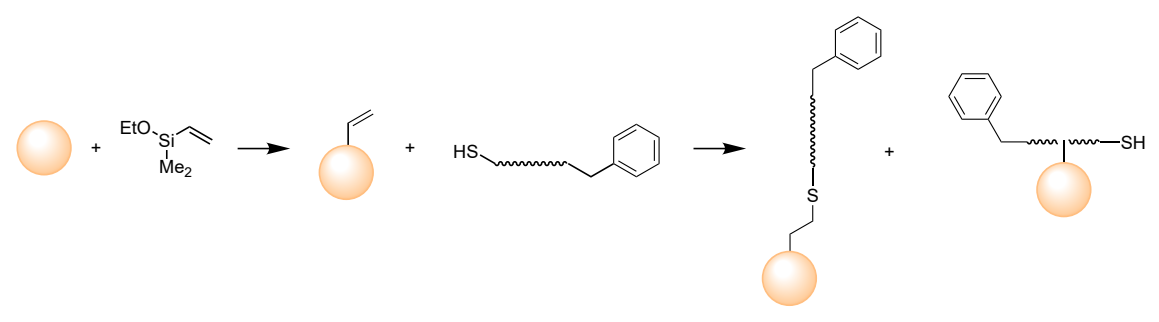

Scheme 3-2: Grafting-to approach for functionalization of silica nanoparticles via thiol-ene click reaction (GT-Click). DMEVS was used as coupling agent. SBR was synthesized using BPTT as RAFT agent and aminolysis was conducted prior to grafting.

illustrated in Scheme 3-2. First, the silica nanoparticles are functionalized with DMEVS (Si-DMEVS). Second, the polymer is synthesized via RAFT polymerization using benzylpropyltrithiocarbonate (BPTT) as RAFT agent. After aminolysis of the polymer it is grafted to Si-DMEVS to yield GT-Click. The thermograms show a two step weight loss for both Si-DMEVS and GT-Click between 250 and $600^{\circ} \mathrm{C}$. Carbon, hydrogen and nitrogen content increase after functionalization (Table 3-1). The increase of nitrogen fraction can be assigned to residues of the aminolysis of the polymer. Analysis of the carbon fraction results in a polymer fraction of $\sim 1.0 \mathrm{wt} \%$ at the particles. Taking the molar masses of the polymers used into account (Table 3-1), the grafting densities of both reaction mechanisms are approximately equal.

\subsubsection{Grafting-from Approach}

Since the grafting-to approaches led to low grafting densities, a second set of samples was prepared, using a grafting-from approach. As mentioned before, the use of silica nanoparticles within the high pressure autoclave is challenging and polymerizations are not always successful due to different reasons. While the synthesis of particles via the Stöber method in the required amount is time consuming, grafting-from samples were synthesized using commercial available silica nanoparticles (GF-Si).

The RAFT agents used for the grafting-from approach are shown in Figure 3-6. Propyl-((trimethoxysilyl)ethylphenylmethyl) trithiocarbonate (PTPT) was chosen as RAFT agent for SBR polymerization in previous works. ${ }^{[11]}$ Activated CDSPA was used, because it can be bound via a monosilyl anchor to the surface, preventing crosslinking of the particles. ${ }^{[12]}$ Addi- 
3 SBR-Functionalized Silica Nanoparticles - Grafting-from versus Grafting-to Approach<smiles>CCCSC(=S)SCc1ccccc1</smiles>

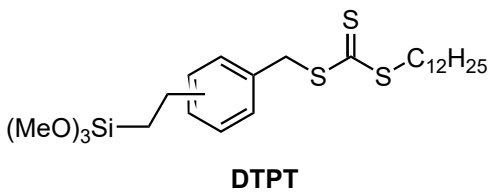<smiles>CCCCCCCCCCCCCCC(C)(CCC(=O)N1CCSC1=S)SC(=S)O[Si](C)(C)CCCN</smiles>

APDEMS + act. CDSPA

Figure 3-6: RAFT agents used for functionalization of silica nanoparticles via the grafting-from approach.

tionally, the dodecyl Z-group at the particle surface leads to hydrophobization of the particles, resulting in enhanced dispersion within the polymerization mixture. Dodecyl-((trimethoxysilyl)ethylphenylmethyl) trithiocarbonate (DTPT) also contains a hydrophobic dodecyl Z-group, but in contrast to CDSPA, the functionalization of particles can be done in a one step process and the synthesis of the RAFT agent is faster.

Figure 3-7 depicts the thermograms of the RAFT agent-functionalized (Si-RAFT) and polymer-functionalized (GF-RAFT) silica nanoparticles. Corresponding weight losses between 250 and $850^{\circ} \mathrm{C}$ are given in Table 3-2. The grafting densities of the RAFT agents are, with respect to their number average molecular mass $\left(\bar{M}_{\mathrm{n}}\right)$, more or less comparable. After polymerization, the amount of bound polymer is different for the samples, ranging from $\sim 4 \mathrm{wt} \%$ for GF-CDSPA to $\sim 16 \mathrm{wt} \%$ for GF-DTPT. The results from EA in Table 3-2 are in good agreement.

Since the cleavage of bound polymer from the particles with hydrofluoric acid did not work, only the free polymers gained from the polymerizations were analyzed (Table 3-2). The results from size-exclusion chromatography (SEC) show, that the number average molecular mass is in the same order of magnitude for all samples. As a consequence, the grafting densities of the three samples are different. An explanation for the huge difference between the sample with CDSPA to the others might be, that the weight loss after functionalization with the RAFT agent is mainly attributed to bound APDMES instead of CDSPA. Meaning, that CDSPA was only bound to some of the amino groups at the particle surface, resulting in a lower grafting 

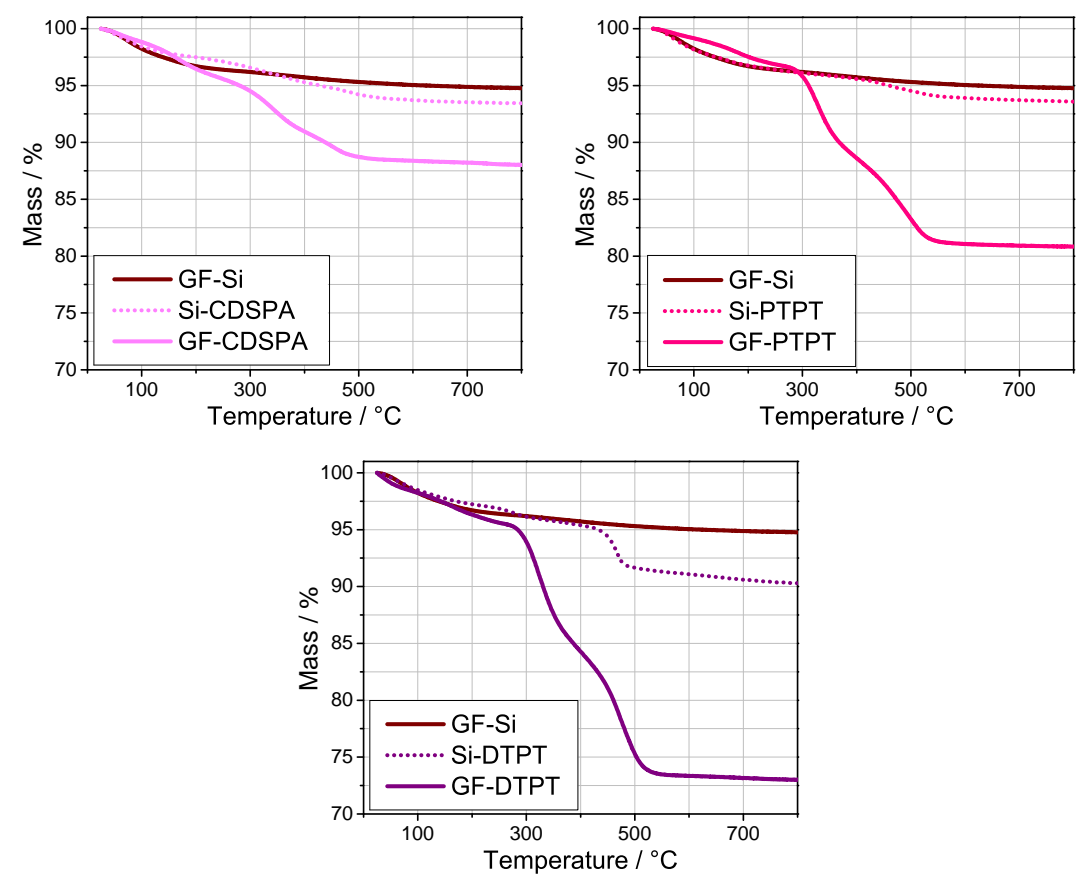

Figure 3-7: Thermograms of non-functionalized (GF-Si), RAFT agent-functionalized (Si-CDSPA, Si-PTPT and Si-DTPT) and polymerfunctionalized silica nanoparticles (GF-CDSPA, GF-PTPT and GF-DTPT) measured under $\mathrm{N}_{2}$ atmosphere.

density of RAFT agent compared to Si-PTPT and Si-DTPT. Nevertheless, using the grafting-from approach, three samples, with higher grafting densities compared to the grafting-to approach, were successfully synthesized. 
3 SBR-Functionalized Silica Nanoparticles - Grafting-from versus Grafting-to Approach

Table 3-2: Weight percentage of carbon, hydrogen and nitrogen measured by EA of non-functionalized (GF-Si), RAFT agent-functionalized (Si-PTPT, Si-CDSPA and Si-DTPT) and polymer-functionalized silica nanoparticles (GF-CDSPA, GF-PTPT and GF-DTPT) and weight loss from TGA analysis measured under $\mathrm{N}_{2}$ atmosphere between 250 and $850{ }^{\circ} \mathrm{C}$. Additionally the number average molecular mass and dispersity of the free polymers are given.

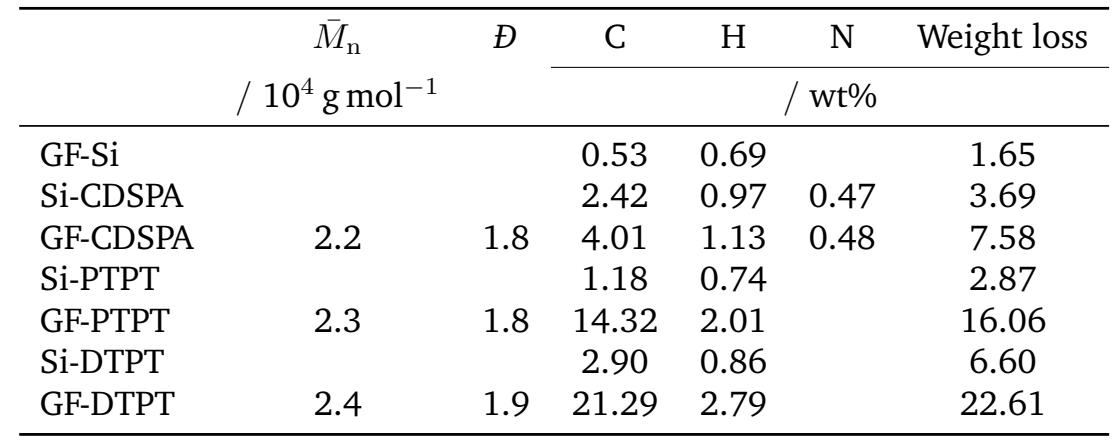

\subsection{Functionalized Silica Nanoparticles as Filler in Tire Compounds}

The functionalized silica nanoparticles (Section 3.3) were intruded into typical tire compounds at the mixing lab of Continental and physical testing was conducted with resulting specimen. The influence of functionalization on the mechanical properties of the compound is analyzed by comparison of the results of the physical tests.

\subsubsection{End Group Modification}

An advantage of RAFT polymerization is, that due to the RAFT agent polymer chains contain sulphur atoms, which could be used for additional crosslinking within the compound. To ensure good dispersion of the filler and other additives, crosslinking should not take place already during mixing but during vulcanization.

Table 3-3 gives an overview of polymer end groups resulting from RAFT polymerization or post-modification. Both, the RAFT end group and the thiol end group, are not able to prevent crosslinking during mixing, since 
3.4 Functionalized Silica Nanoparticles as Filler in Tire Compounds

Table 3-3: Overview of possible polymer end groups resulting from RAFT polymerization or post-modification.

\begin{tabular}{cll}
\hline Structure & Name & Properties \\
\hline S & RAFT end group & $\begin{array}{l}\text { Might be cleaved off during mixing. } \\
\text { The influence of the leaving group } \\
\text { within the compound is unknown. }\end{array}$ \\
& Thiol & $\begin{array}{l}\text { Crosslinking during mixing possible. } \\
\text { Protective group for sulphur during } \\
\text { mixing. }\end{array}$ \\
& Cleaved off during vulcanization. \\
$\mathrm{P}_{\mathrm{n}}-\mathrm{S}_{\mathrm{R}_{\mathrm{R}}}$ & Thioether & $\begin{array}{l}\text { Protective group for sulphur during } \\
\text { mixing and vulcanization. }\end{array}$ \\
\hline
\end{tabular}

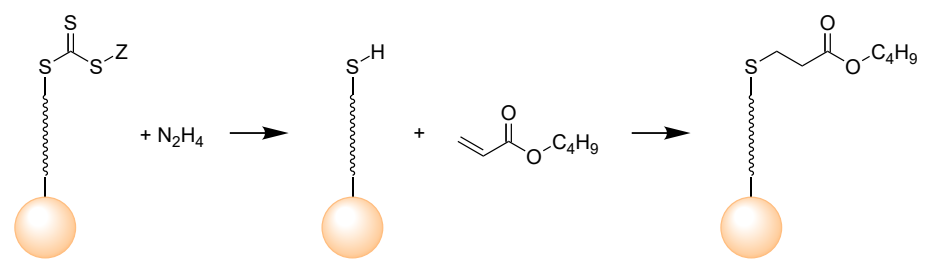

Scheme 3-3: End group modification of surface bound RAFT polymer.

the sulphur atom is not protected under mixing conditions. The thioester is a typical protective group for sulphur during mixing and can be found as part of many silanes used in tire industry. ${ }^{[80]}$ In comparison to the other end groups mentioned, its synthesis is more challenging, especially when it comes to the purification in presence of nanoparticles. Thioether also act as protective group during mixing, but also during vulcanization. The synthesis as well as the purification in presence of nanoparticles is simple and yields $>95 \%$ are obtained. [81]

For this study, the use of thioether end groups was chosen due to several reasons. First, the dispersion of the functionalized silica nanoparticles is not affected by undesired crosslinking during mixing. Second, the synthesis ensures complete conversion. The end group modification of functionalized 
3 SBR-Functionalized Silica Nanoparticles - Grafting-from versus Grafting-to Approach

silica nanoparticles from Section 3.3 was conducted via aminolysis, followed by addition of $n$-butyl acrylate (Scheme 3-3).

\subsubsection{Mixing of the Compounds and Production of the Test Specimens}

For each silica sample, three compounds with different amounts of silica, given in parts per hundred rubber (phr), between 35 and 80 phr were prepared. As reference samples compounds with the non-functionalized silica nanoparticles GT-Si and GF-Si as well as with a standard silica used by Continental (VN3) were mixed and tested too. Besides the silica particles, some reference samples also contained silane (S) or liquid butadiene rubber (LBR, in the denotation of the samples referred to as L). LBR is a low-molecular weight polybutadiene with a high ratio of 1,2-units (Subsection 3.2), which show stronger interaction with the silica surface than 1,4-units. [82] The strong interaction of LBR with silica enhances particle dispersion within in the compound and is therefore used in addition to silane in SBR filled compounds. [83]

The mixing of the samples was conducted in a four step process in an internal mixer. The mixing temperature was controlled by the rotor speed ${ }_{i i}^{i i}$ At first a master batch (Table 3-4) containing rubbers, softener, antioxidants and activators for vulcanization was prepared. In the second step, $134 \mathrm{phr}$

\footnotetext{
${ }^{i i}$ Detailed information about the used materials and mixing conditions, like time and temperature, can not be given due to non-disclosure of internal company knowledge.
}

Table 3-4: Composition of the master batch and the final batch used for mixing of the compounds.

\begin{tabular}{lrr}
\hline & \multicolumn{2}{c}{ Amount / phr } \\
\cline { 2 - 3 } & Master batch & Final batch \\
\hline Rubber & 90 & 10 \\
Softener & 35 & \\
Antioxidants & 4 & \\
Activators & 5 & 2 \\
Sulfur & & 4 \\
Accelerators & & \\
\hline
\end{tabular}


3.4 Functionalized Silica Nanoparticles as Filler in Tire Compounds

Table 3-5: Quantities of silica, silane and LBR used for compound mixing. Each compound was mixed using $134 \mathrm{phr}$ of the master batch and $16 \mathrm{phr}$ of the final batch resulting in 27 samples.

\begin{tabular}{|c|c|c|c|}
\hline & Si / phr & Silane / phr & LBR / phr \\
\hline \multicolumn{4}{|l|}{ References: } \\
\hline \multirow{3}{*}{$\mathrm{VN} 3+\mathrm{S} / \mathrm{GF}-\mathrm{Si}+\mathrm{S}$} & 60 & 4.32 & \multirow{11}{*}{$8,16,24$} \\
\hline & 70 & 5.04 & \\
\hline & 80 & 5.76 & \\
\hline VN3+L/GT-Si+L & 70 & & \\
\hline GF-Si & $50,60,70,80$ & & \\
\hline GT-Si & $35,50,60,70,80$ & & \\
\hline \multicolumn{3}{|c|}{ Samples: } & \\
\hline$\overline{\text { GT-Subs }}$ & $35,60,70$ & & \\
\hline GT-Click/GF-DTPT & $50,60,70$ & & \\
\hline GF-PTPT & $60,70,80$ & & \\
\hline GF-CDSPA & $60,70,75$ & & \\
\hline
\end{tabular}

of the master batch were mixed with silica and in some cases silane or LBR (Table 3-5). In case of functionalized silica nanoparticles the amount of added particles (Table C.1) was calculated using the residual mass taken from TGA measurements. The final batch (Table 3-4) prepared in the third step contains rubbers, sulphur and accelerators for the vulcanization. At least $16 \mathrm{phr}$ of the final batch were added to each compound and mixed. After resting for several days a specimen of each compound was formed and cured at $160^{\circ} \mathrm{C}$ for $10 \mathrm{~min}$.

\subsubsection{Results of Physical Testing}

The results of physical tests can be divided into two groups: one giving information about the composition and quality of the sample (torque, TGA, bound sulphur content, density and crosslink distance) and the other revealing the mechanical properties (hardness, rebound, tensile testing, dynamic mechanical analysis (DMA) and abrasion). For each sample 13 tests using different types of devices and measurement modes have been performed. In the following, only selected results will be presented and discussed in 
3 SBR-Functionalized Silica Nanoparticles - Grafting-from versus Grafting-to Approach

order to point out interesting and characteristic properties of the presented samples ${ }_{\text {iii }}^{\text {ii }}$ Additional results are given in Tables D.1 D.5.

\section{Torque}

The torque was measured using a rubber process analyzer. Within the heated chamber of the rheometer, the uncured sample is placed between two horizontal plates under constant pressure. The lower plate oscillates with constant frequency $(1 \mathrm{~Hz})$ and the upper plate contains a torque sensor, measuring the torque required for oscillation. This way, the progress of vulcanization is monitored as plot of torque versus time (rheogram).

Figure 3-8(a) shows the rheograms of the samples containing VN3 plus silane $(\mathrm{VN} 3+\mathrm{S})$ with different loadings of silica at $160^{\circ} \mathrm{C}$. Typically, the curve consists of three sections, which are determined by the composition of the compound. In the beginning the torque is low and more or less constant. When curing sets in, the torque strongly increases. Knowledge of this time slot is of great importance for tire production, since the inner and outer parts of the tire are heated up with different speed. It can be controlled e.g. through the amount of vulcanization activators and accelerators within the compound. Curing is finished, when the torque reaches a maximum value. Depending on the sample, this optimum state-of-cure can be identified more or less easily. Instead of reaching a plateau, some samples show a slightly continuous increase of torque (marching modulus) or even a revision of torque.

The comparison of the rheograms of VN3 +S samples (Figure 3-8(a)) show, that the torque increases with silica content due to its reinforcing properties. The sample filled with $60 \mathrm{phr}$ silica reaches a plateau after $10 \mathrm{~min}$ and the samples with 70 and $80 \mathrm{phr}$ silica show a marching modulus.

While the addition of LBR enhances silica dispersion, it reduces the crosslink density and therefore the torque decreases with increasing amount of LBR. [83] Figure 3-8(b) shows the rheograms of VN3+S 70 and samples of VN3 filled with $70 \mathrm{phr}$ silica and different amounts of LBR (VN3+L). The maximum torque of the sample with the lowest LBR content is comparable to the silane sample. As expected, the samples with 16 and $24 \mathrm{phr}$ silica have lower torque, but not within the right order. This is an example, of how the torque can give information about the quality of a sample. From

\footnotetext{
iiiTest specifications as well as internal knowledge about the analysis of the tests were provided by Continental.
} 

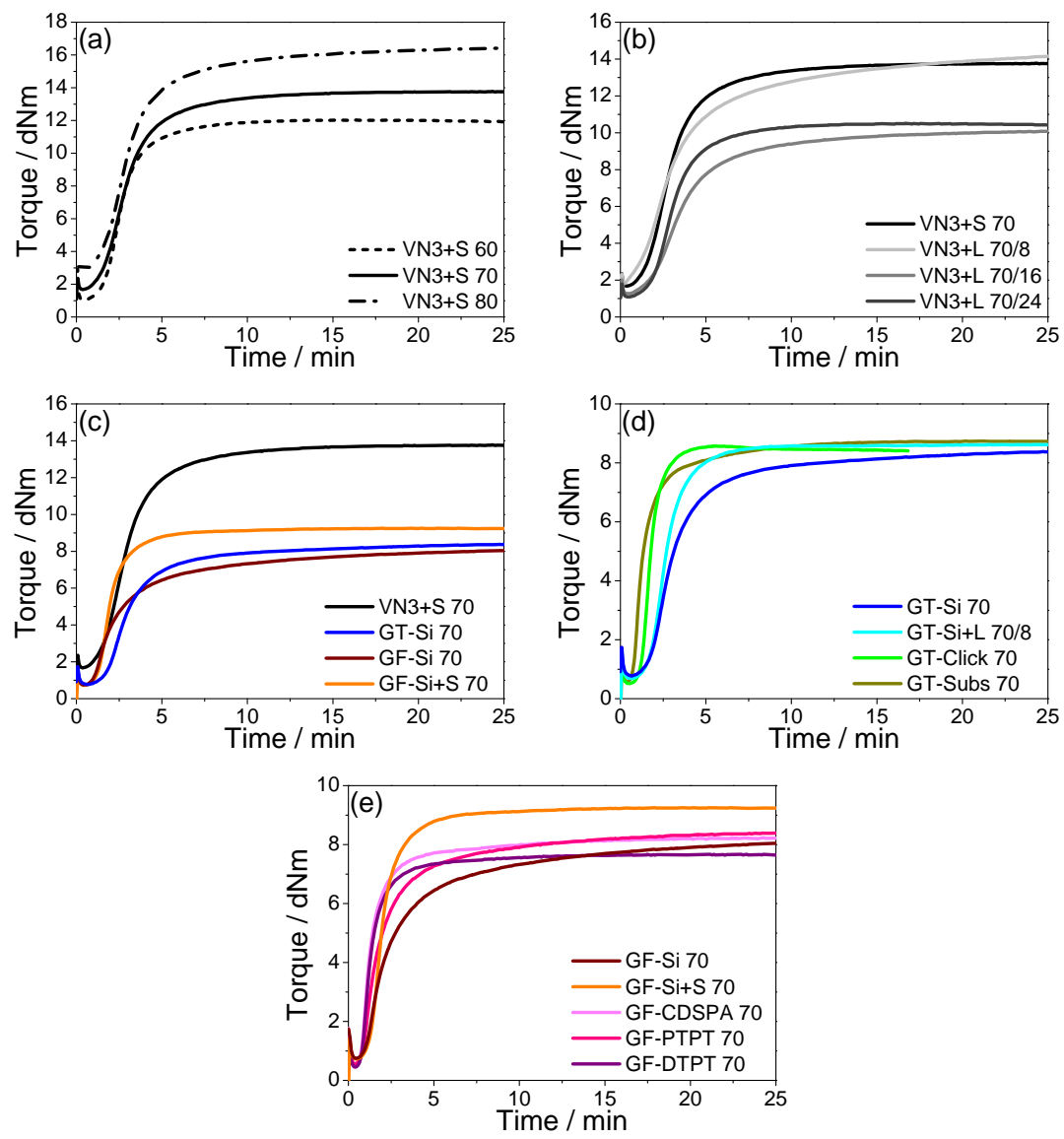

Figure 3-8: Rheograms of the compounds containing $70 \mathrm{phr}$ silica at $160^{\circ} \mathrm{C}$.

the rheograms it can be concluded, that either the sample with 16 or $24 \mathrm{phr}$ is flawed, e.g. due to insufficient mixing. Other test results of these samples need to be analyzed carefully and could give more information about which sample should not be considered for further analysis and comparison.

The rheograms of GF-Si, GF-Si+S and GT-Si with $70 \mathrm{phr}$ silica are depicted in Figure 3-8(c). The torque is only about half of the value reached with VN3, showing that GF-Si and GT-Si are less reinforcing. This demonstrates 
3 SBR-Functionalized Silica Nanoparticles - Grafting-from versus Grafting-to Approach

the influence of shape, surface activity and particle size on the interactions with the rubber matrix. As expected, the addition of silane in case of the GF-Si increases the torque. All samples either reach a plateau or show a slight marching modulus.

The samples with $70 \mathrm{phr}$ of silica functionalized via the grafting-to approach show both slightly higher torque compared to GT-Si 70 (Figure 3-8(d)) and approximately the same value as the reference with additional $8 \mathrm{phr}$ LBR. Also, curing is faster for both functionalized samples. In contrast to all other samples GT-Click 70 shows revision but after $10 \mathrm{~min}$ the torque is at a constant level.

Comparison of the $70 \mathrm{phr}$ samples containing silica functionalized via the grafting-from approach with GF-Si with and without silane shows, that after $10 \mathrm{~min}$, their torque is in between the references (Figure 3-8(e)). As for the results of the grafting-to samples, an effect of functionalization can be seen, but the reinforcing effect is less than by the usage of silane.

The analysis of the torque measurements point out that both reference silica GT-Si and GF-Si are less reinforcing than VN3. This has to be kept in mind for the further discussion of the results of the physical tests. Additionally, the results show an increase of the torque of functionalized silica nanoparticles compared to the non-functionalized references.

\section{Crosslink Distance}

The crosslink distance is a measure for network strength within the cured compound. Smaller distances means stronger networks, resulting in enhanced reinforcement of the mechanical properties. It can be adjusted through the vulcanization system (sulphur, activators and accelerators) and in case of filled compounds, it is also influenced by the structure and surface chemistry of the filler. [84]

Figure 3-9(a) depicts the crosslink distance depending on the silica content of grafting-to samples and corresponding references. With increasing silica content the crosslink distance decreases in case of VN3+S. This behavior is typical for SBR compounds filled with silanized silica. ${ }^{[85]}$ The crosslink distance of GT-Si is larger compared to VN3+S and no trend with silica loading can be observed. As mentioned before, the addition of LBR increases the crosslink distance, which can be observed for both types of silica, VN3 and GT-Si, containing $8 \mathrm{phr}$ LBR. As stated in literature, this is due to adsorption of vulcanization chemicals onto the surface. ${ }^{83]}$ The compounds filled with silica functionalized via the grafting-to approach have a crosslink distance of 

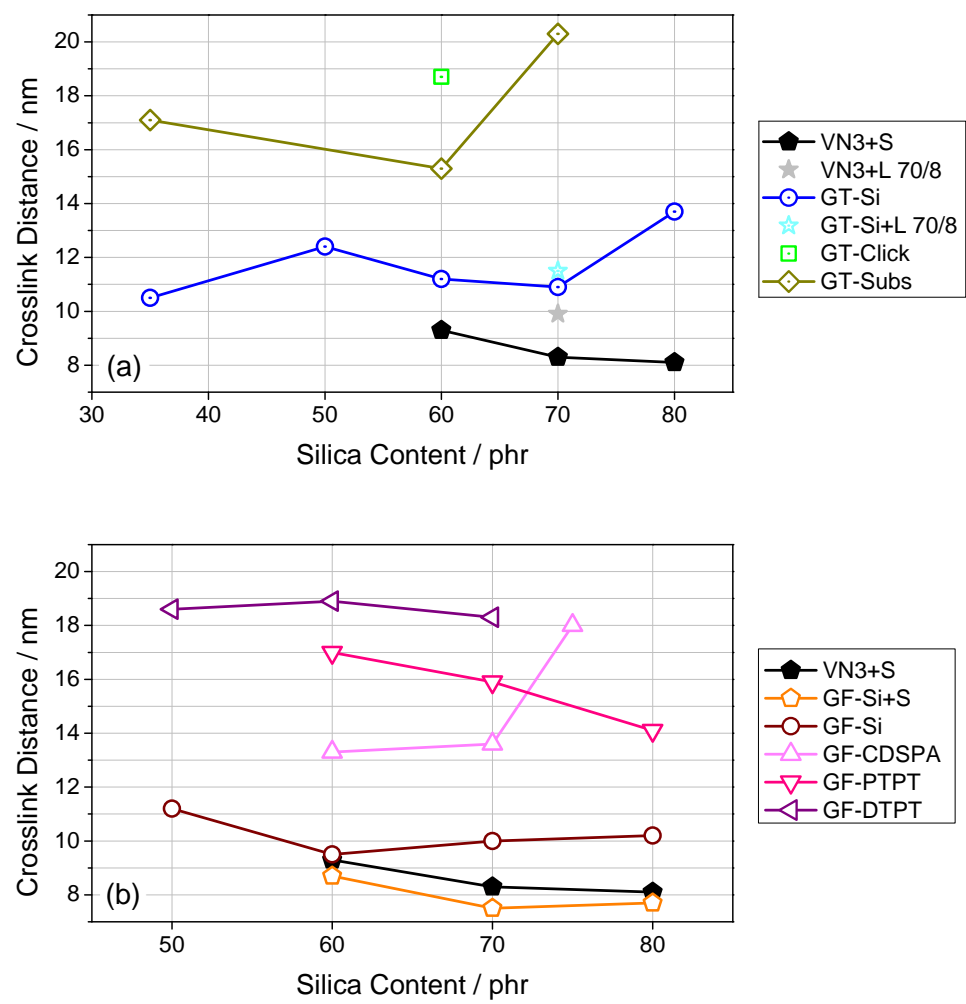

Figure 3-9: Crosslink distance depending on the silica content. Data points have been connected for better overview.

nearly twice the values of the reference samples and again no trend can be observed with silica content. Due to the additional surface bound polymer within the compound, the sulphur-to-polymer ratio is lower compared to the references. But since this is also the case for samples containing LBR, this alone can not explain the huge difference of crosslink distance observed.

Similar observation can be made for the grafting-from samples and corresponding references (Figure 3-9(b)). The crosslink distance of the silanized reference silica $\mathrm{VN} 3+\mathrm{S}$ and $\mathrm{GF}-\mathrm{Si}+\mathrm{S}$ have more or less the same values and it decreases with increasing silica content. The values for GF-Si without 
3 SBR-Functionalized Silica Nanoparticles - Grafting-from versus Grafting-to Approach

silane are a little higher and comparable to GT-Si in Figure 3-9(a). The functionalization of silica particles again results in larger crosslink distance. Thereby, the increase is proportional to the amount of surface bound polymer, ranging from GF-CDSPA ( $4 \mathrm{wt} \%$ ) to GF-DTPT (16 wt $\%$ ). This supports the assumption, that the lowered sulphur-to-polymer ratio is one reason for the increase of crosslink distance. Again, no relationship between functionalized silica content and crosslink distance can be found, since all three samples behave different.

The results of crosslink distance analysis show, that in case of compounds filled with functionalized silica, the network is weaker compared to reference samples. This has to be taken into account, when the results of mechanical testing will be discussed.

\section{Hardness and Rebound}

The hardness was tested according to Shore A (ISO 868). A pressure foot is dent into the specimen with a given force and the depth of indentation, which is proportional to the viscoelastic properties of the material, is measured. Ranging from 0 to $2.5 \mathrm{~mm}$, the depth of indentation is split in 100 so-called Shore units (ShA). ${ }^{[3,86]}$ The rebound was measured in a pendulum impact test (DIN 53512:2000-04). A pendulum is dropped from a horizontal position onto the specimen and its rebound, which is proportional to the energy absorbed in the material, is detected optically. The values are given in percentage to the initial height of the pendulum. ${ }^{[3,86]}$ Both hardness and rebound are measured at room temperature (RT) and $70^{\circ} \mathrm{C}$. [3] These temperatures refer to the operating temperatures of a tire, where RT corresponds to braking behaviour and $70^{\circ} \mathrm{C}$ represents the rolling resistance. ${ }^{[3.57]}$ In filled, cured compounds the hardness RT and the rebound $70^{\circ} \mathrm{C}$ correlate to each other. As rule of thump, the addition of 2 phr silica leads to an increase of $1 \mathrm{ShA}$ at RT and the decrease of $1 \%$ rebound at $70^{\circ} \mathrm{C}$. [86]

The increase of hardness RT with silica content can be seen in Figure 3-10. In both cases for the grafting-to (a) and the grafting-from (b) approach, all samples show a lower hardness compared to VN3+S. This is in accordance to the results discussed already. GT-Subs and GT-Click show no significant difference compared to the reference silica GT-Si. The grafting-from samples have a higher hardness than the non-functionalized reference silica GF-Si and in case of GF-DTPT, it is even higher compared to the silanized silica. This is outstanding when taking the crosslink distance into account. With increasing crosslink distance hardness typically decreases. As the compounds 

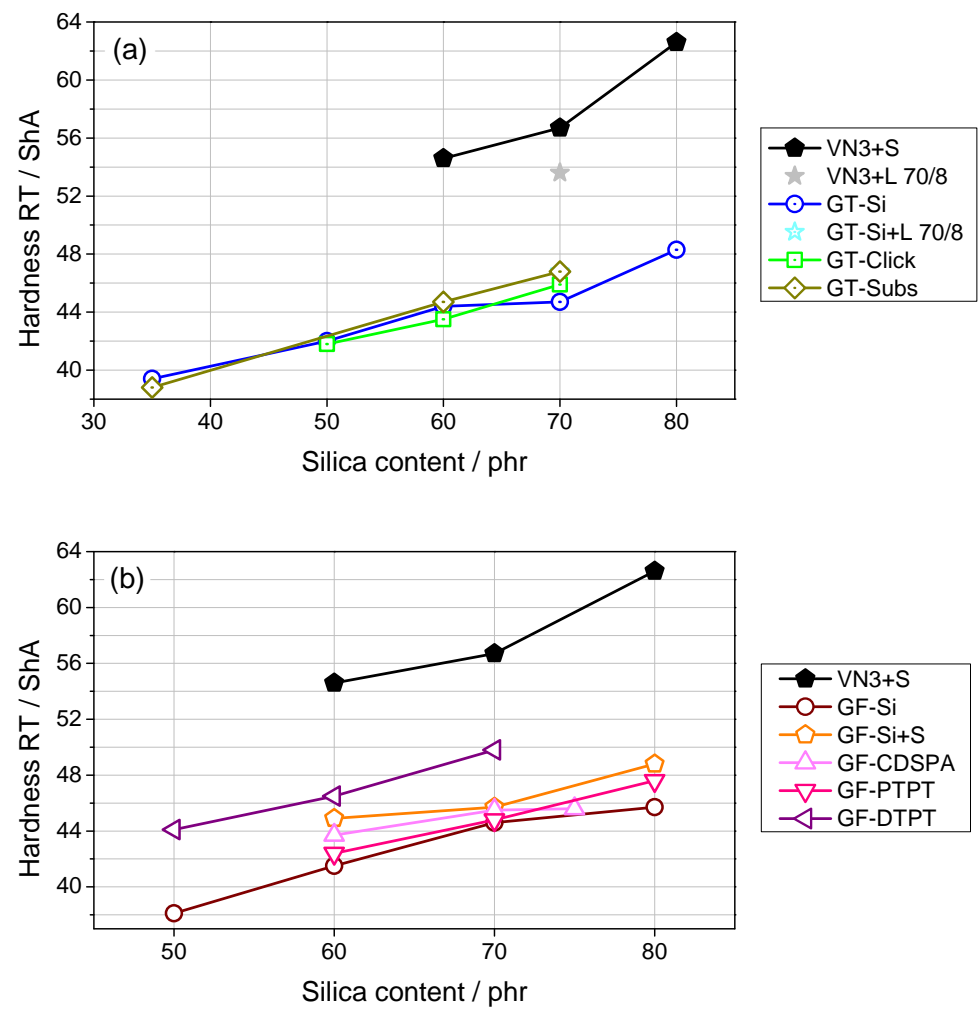

Figure 3-10: Hardness RT depending on silica content. Data points have been connected for better overview.

containing GF-DTPT have a high crosslink distances and therefore poorly developed networks, the reinforcing effect must have another origin.

The difference of the rebound at $70^{\circ} \mathrm{C}$ and $\mathrm{RT}$ is a measure of tire performance and should be as high as possible. Good rolling resistance requires high rebound at $70^{\circ} \mathrm{C}$ and good braking behavior is ensured by low rebound at RT. For compounds filled with $\mathrm{VN} 3+\mathrm{S}$ the difference increases with silica content (Figure 3-11), whereas for all the other samples the value either decreases or is more or less constant. At a silica content of $70 \mathrm{phr}$, the 
3 SBR-Functionalized Silica Nanoparticles - Grafting-from versus Grafting-to Approach
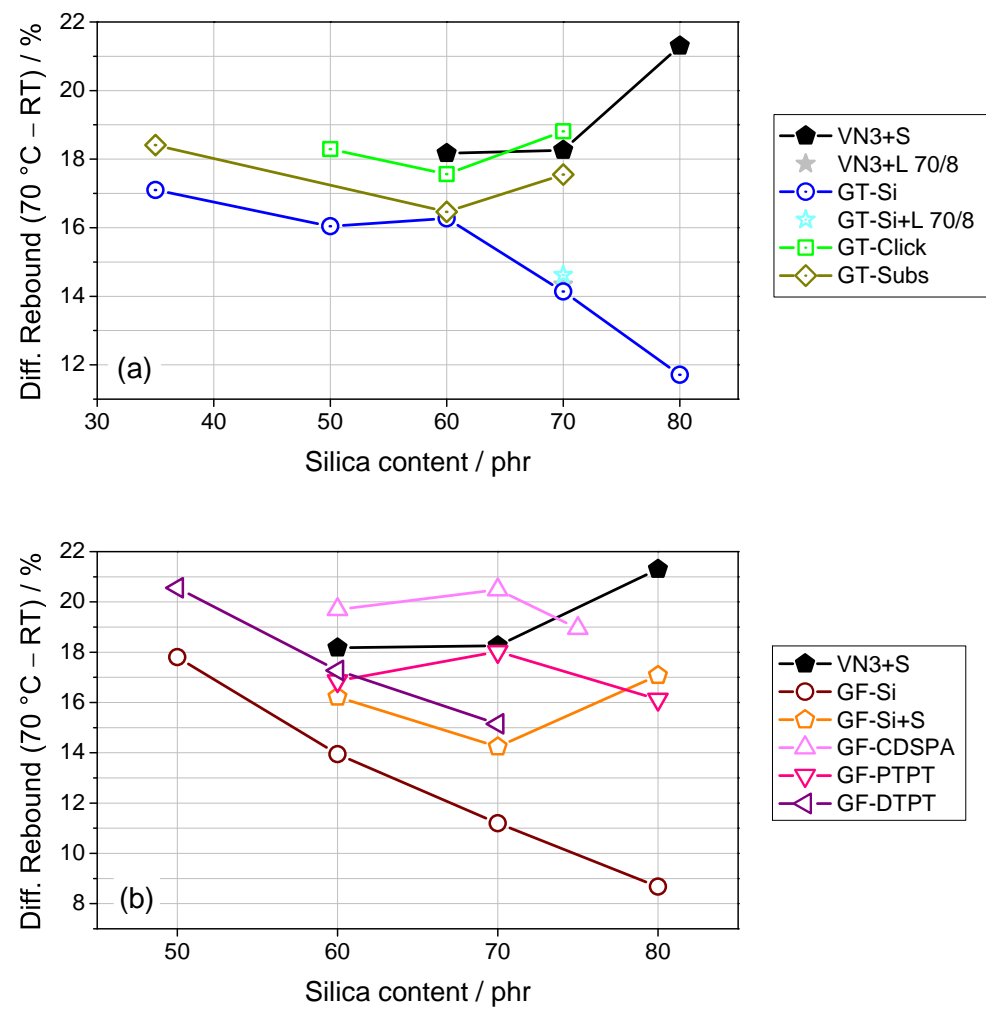

Figure 3-11: Difference of rebound $70^{\circ} \mathrm{C}$ and RT depending on the silica content. Data points have been connected for better overview.

addition of LBR to GT-Si (Figure 3-11(a)) does not have an effect, but functionalization leads to an increase, resulting in values close to VN3+S. The results of grafting-from samples in Figure 3-11(b) show, that the functionalization leads to higher values than the addition of silane. The difference of GF-CDSPA compounds is even higher than that of VN3+S. Interestingly, with regard to the amount of surface bound polymer GF-CDSPA is comparable to GT-Subs and GT-Click. Overall, the results show a positive effect of the functionalization on the rebound. 


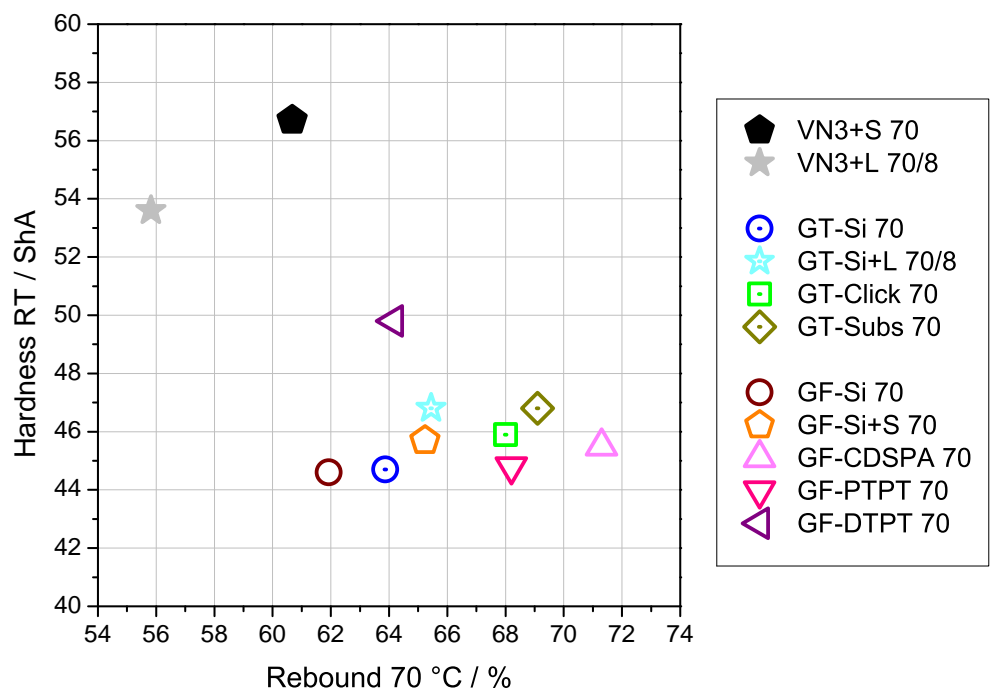

Figure 3-12: Hardness RT versus rebound at $70{ }^{\circ} \mathrm{C}$ of compounds filled with 70 phr silica.

From the plot of hardness RT against rebound $70^{\circ} \mathrm{C}$ of compounds filled with 70 phr silica in Figure 3-12 it can be seen that except for GF-DTPT the functionalization of silica nanoparticles does have a greater impact on rebound than on hardness. This shows, that functionalization might be a powerful tool to adjust both hardness and rebound, e.g. by the choice of grafting density or chain length of the surface bound polymer.

\section{Tensile Testing}

Tensile tests were performed at RT (DIN 53594). The specimen is stretched with constant speed until tearing. The stress at elongations of 100, 200 and $300 \%$ as well as the tensile strength and the tear elongation are characteristic values from tensile testing.

The stress at 100\% (M100) and 300\% (M300) elongation of compounds filled with grafting-to samples and corresponding reference silica are given in Figure 3-13. As could be expected, the M100 values are comparable to the results of hardness RT (Figure 3-10) and only little effect of the func- 
3 SBR-Functionalized Silica Nanoparticles - Grafting-from versus Grafting-to Approach

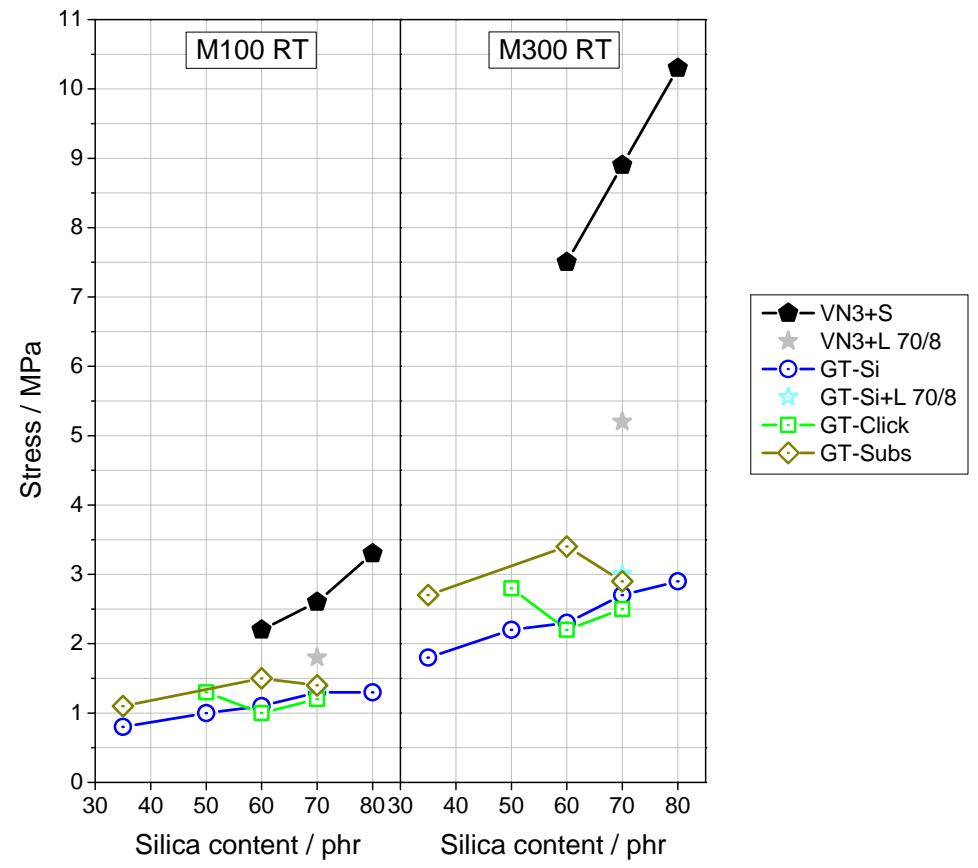

Figure 3-13: Stress at 100 (left) and 300\% (right) elongation at RT of grafting-to samples and corresponding references. Data points have been connected for better overview.

tionalization as well as of the addition of LBR can be observed. At $300 \%$ elongation the filler-filler network is broken down, so the M300 value is a measure of polymer-filler interactions and strength of the polymer network. ${ }^{[87]}$ Even though the crosslink distance of GT-Subs is twice that of GT-Si, the M300 values are increased significantly. This indicates the improvement of polymer-filler interactions through the functionalization of silica nanoparticles.

Similar observation can be made for the compounds filled with graftingfrom samples in Figure 3-14. Like the addition of silane, the functionalization of silica nanoparticles leads to an increase of M100 and M300. The M100 values of GF-DTPT are even higher compared to silanized reference silica. This 


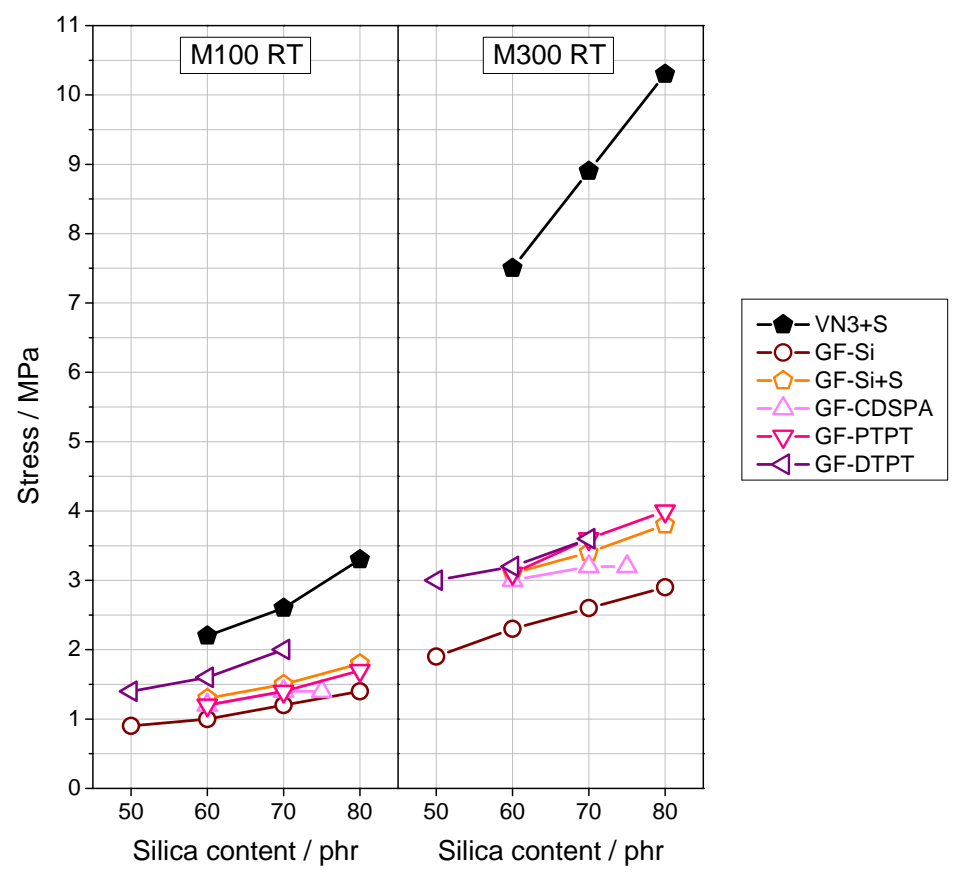

Figure 3-14: Stress at 100 (left) and 300\% (right) elongation at RT of grafting-from samples and corresponding references. Data points have been connected for better overview.

shows that filler-filler interactions are reduced by the surface bound polymer while simultaneously the polymer-filler interactions are enhanced. The latter is also reflected in the comparison of M300. Despite the weak polymer network, due to high crosslink distances, the M300 values of grafting-from samples are equal to GF-Si+S.

Overall, the result of tensile testing show that the functionalization of silica nanoparticles reduces the filler-filler interactions and enhances the polymer-filler interactions. On the one hand, the non-polar surface bound polymer shields the silica surface, which reduces the interactions between the hydrophilic particles. On the other hand, the interactions of the particles 
3 SBR-Functionalized Silica Nanoparticles - Grafting-from versus Grafting-to Approach

with the matrix polymer are increased through the entanglement of the surface bound polymer chains with the matrix.

\section{Dynamic Mechanical Analysis}

The dynamic mechanical analysis (DMA) was conducted using an eplexor. The storage modulus and the $\tan \delta$ are measured under compression, either in temperature or strain sweep mode.

The Payne effect is of great importance for tire compounds (see Subsection 2.3.2). ${ }^{[57]}$ It describes the strain-dependent storage modulus, which is a measure for filler-filler interactions. The weaker these interactions are, the lower is the slope of the curve. Figure 3-15 depicts the strain-dependent storage modulus of the compounds filled with $70 \mathrm{phr}$ silica. Surprisingly, the storage modulus of GT-Si is higher than that of both functionalized samples, as well as of the sample with additional LBR (Figure 3-15(a)). This is contrary to the results of torque measurement and tensile testing. While the addition of LBR does not have an effect on the slope of the curve, for GT-Subs the Payne effect is slightly reduced.

The picture is different for compounds filled with grafting-from silica in Figure 3-15(b). As expected, the addition of silane increases the storage modulus, but no significant reduction of the Payne effect can be observed. The storage modulus and the Payne effect are not affected in case of GF-PTPT and GF-CDSPA. In case of GF-DTPT the storage modulus is a little higher than that of silanized silica, but the Payne effect is stronger. This somehow disagrees with the results of tensile testing (Figure 3-14), which indicated reduced filler-filler interactions for GF-DTPT compounds.

Another indicator for tire performance is $\tan \delta$ from DMA measurements at constant force (see Subsection 2.3.2). To ensure good wet grip and skid resistance, a high $\tan \delta$ at temperatures between -20 to $0^{\circ} \mathrm{C}$ is necessary. A low $\tan \delta$ around 50 to $80^{\circ} \mathrm{C}$ reduces rolling resistance. [57]

At both temperatures, -15 and $70^{\circ} \mathrm{C}$, the functionalization of silica via the grafting-to approach improves $\tan \delta$ compared to GT-Si (Figure 3-16). In contrast, the addition of LBR does not affect $\tan \delta$.

The results of grafting-from samples in Figure 3-17 show an enhancement of $\tan \delta$ by the addition of silane at both temperatures with respect to the above-mentioned criteria. Only little effect can be observed for GF-DTPT, while GF-PTPT and GF-CDSPA improve $\tan \delta$ significantly. At $-15^{\circ} \mathrm{C}$ their $\tan \delta$ values are even higher compared to silanized reference silica. 

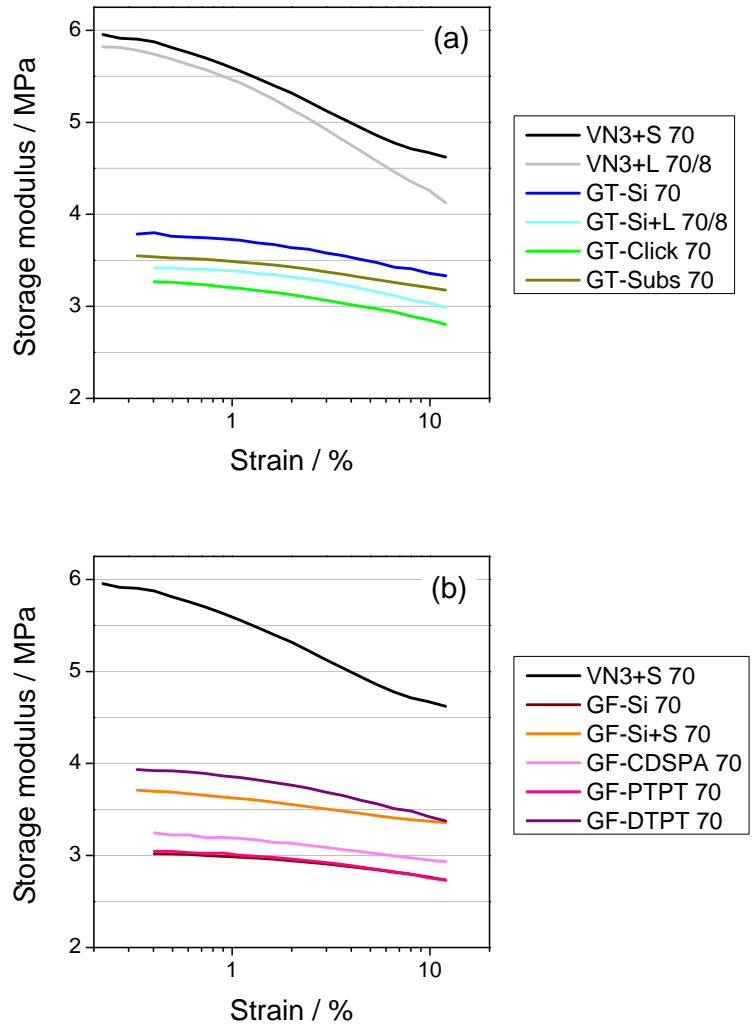

Figure 3-15: Strain-dependent storage modulus of samples filled with $70 \mathrm{phr}$ silica, measured with an eplexor in strain sweep mode at $55^{\circ} \mathrm{C}$.

The results may allow to draw some conclusions about the influence of the way of silica functionalization on filler-filler and polymer-filler interactions. While LBR does only interact with the silica surface via physisorption, in case of silane and both grafting approaches (macro)molecules are bound chemically. The incorporation of silica into the polymer network is improved through the covalent bond. 

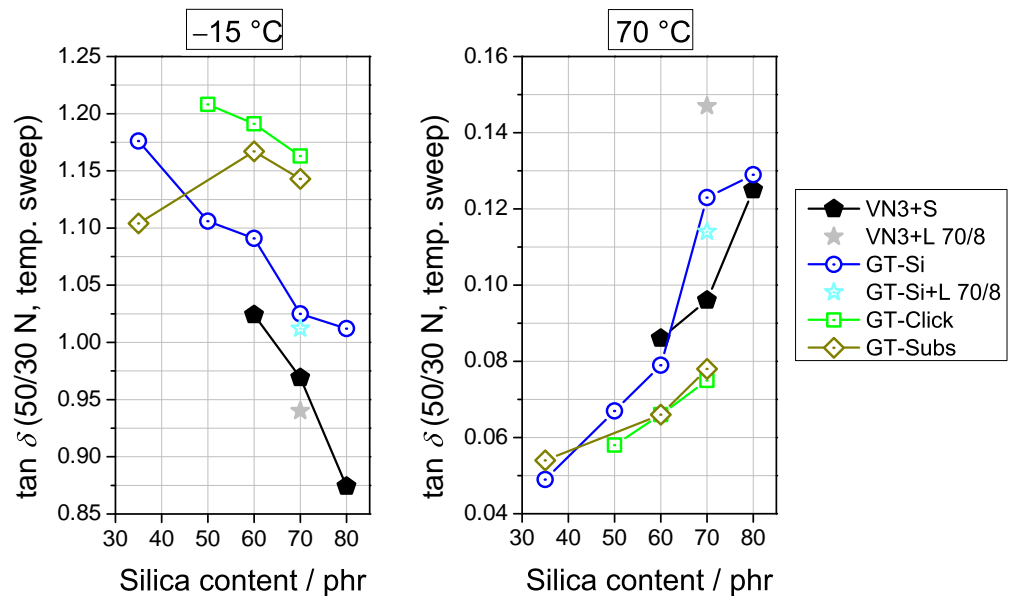

Figure 3-16: Tan $\delta$ at $-15^{\circ} \mathrm{C}$ (left) and $70^{\circ} \mathrm{C}$ (right) of grafting-to samples and corresponding references, measured with an eplexor in temperature sweep mode under constant force $(50 / 30 \mathrm{~N})$. Data points have been connected for better overview.
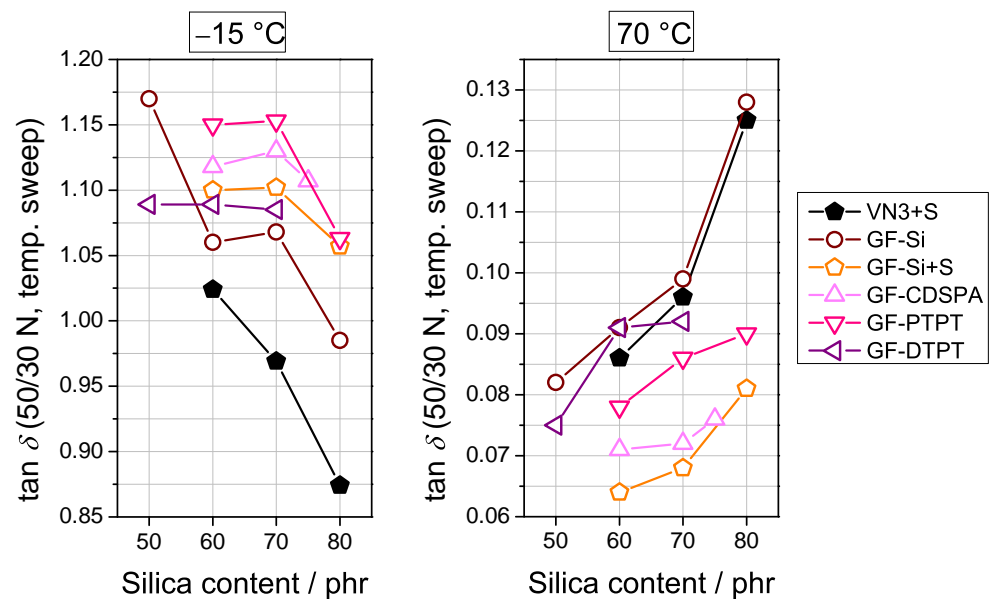

Figure 3-17: Tan $\delta$ at $-15^{\circ} \mathrm{C}$ (left) and $70^{\circ} \mathrm{C}$ (right) of grafting-from samples and corresponding references, measured with an eplexor in temperature sweep mode under constant force $(50 / 30 \mathrm{~N})$. Data points have been connected for better overview. 


\begin{abstract}
Abrasion
Abrasion was tested using a rotary drum abrader (DIN ISO 4649). This test method measures the volume loss due to the abrasive action of rubbing a test piece over a specified grade of abrasive sheet. The abrasion is an indicator for polymer-filler interactions and strength of the polymer network. With regard to durability and environmental pollution, it should be as low as possible.

Since both silica used for the grafting approaches, GT-Si and GF-Si, are less reinforcing than VN3, the abrasion of corresponding compounds is higher (Figure 3-18). As seen before, the crosslink distance of compounds filled with functionalized silica nanoparticles is high compared to the reference samples. This could lead to a reduction of network strength. Nevertheless, the abrasion is equal or less compared to GT-Si and GF-Si. This again indicates an improvement of polymer-filler interactions by the functionalization of silica surface, resulting in reinforcement of the compound. Additionally, in Figure 3-18(b) it can be seen that this improvement is similar or even better compared to the use of silane. This shows that the polymer-filler interactions are not only enhanced through the incorporation of the silica particles into the polymer network during vulcanization but are also improved by the entanglement of the surface bound polymer chains with the matrix polymer.
\end{abstract}


3 SBR-Functionalized Silica Nanoparticles - Grafting-from versus Grafting-to Approach
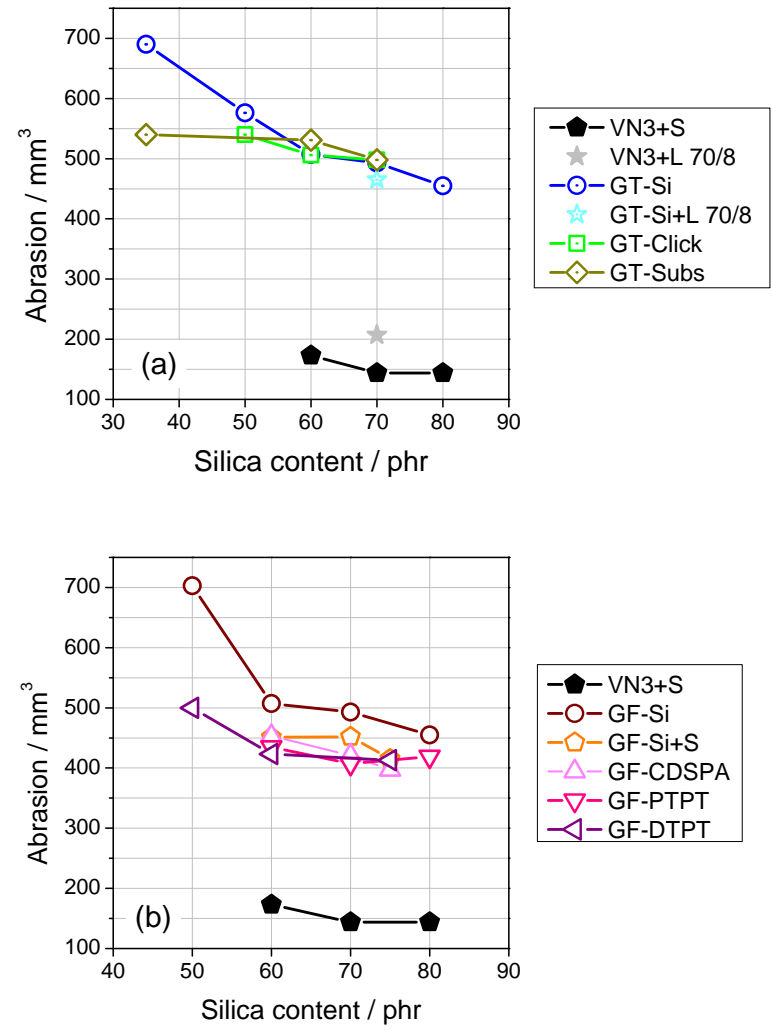

Figure 3-18: Abrasion depending on the silica content. Data points have been connected for better overview.

\subsection{Conclusion and Future Perspectives}

Silica nanoparticles functionalized with SBR via grafting-to and graftingfrom approach were used as filler in tire compounds and the results of physical tests were discussed. Two strategies have been used for the graftingto approach. In two step processes, silica nanoparticles were functionalized first with small molecules and afterwards SBR was grafted via nucleophilic substitution or thiol-ene click reaction onto the surface. Both synthetic 
Table 3-6: Summarizing evaluation of the results of physical tests of compounds filled with silica nanoparticles functionalized via grafting approaches. Evaluation criteria are shown below. The results of VN3 samples are not taken into account, so the physical properties are only compared to those of the corresponding reference silica GT-Si and GF-Si. Samples appear in ascending order of the amount of grafted polymer.

\begin{tabular}{ccccccc}
\hline Worse & $<$ & Si & $<$ & Silane & $<$ & Better \\
& - & 0 & + & ++ & +++ & \\
\hline
\end{tabular}

\begin{tabular}{lccccc}
\hline & GT-Click & GT-Subs & GF-CDSPA & GF-PTPT & GF-DTPT \\
\hline Hardness RT & 0 & 0 & + & + & +++ \\
Diff. Rebound & +++ & ++ & +++ & ++ & +++ \\
(70 $\left.{ }^{\circ} \mathrm{C}-\mathrm{RT}\right)$ & & & & & \\
Hardness RT vs. & +++ & +++ & +++ & +++ & +++ \\
Rebound 70 ${ }^{\circ} \mathrm{C}$ & & & & & \\
M100 RT & 0 & + & + & + & +++ \\
M300 RT & 0 & ++ & + & ++ & ++ \\
$E^{\prime} \quad(0.15-8 \%)$ & 0 & + & + & + & - \\
$55^{\circ} \mathrm{C}$ & & & & & \\
Tan $\delta\left(-15^{\circ} \mathrm{C}\right)$ & +++ & ++ & +++ & +++ & + \\
$\operatorname{Tan} \delta\left(70^{\circ} \mathrm{C}\right)$ & + & + & ++ & + & 0 \\
$\operatorname{Abrasion}$ & 0 & 0 & +++ & +++ & +++ \\
\hline
\end{tabular}

strategies led to low amount of grafted polymer, with mass fractions below $2 \mathrm{wt} \%$ determined by EA. The grafting-from approach was conducted using three RAFT agents (PTPT, CDSPA and DTPT) for SBR polymerization within a high-pressure autoclave. The amount of grafted polymer, determined by TGA, ranged from $4 \mathrm{wt} \%$ for GF-CDSPA to $16 \mathrm{wt} \%$ for GF-DTPT.

Before mixing of the compounds, end group modification of the surface bound polymer was conducted using aminolysis and reaction with $n$-butyl acrylate. Resulting thioether end groups act as protective group for the remaining sulphur atom. For each functionalized silica, three compounds with different filler loading were prepared.

A summarizing evaluation of the physical test results is given in Table 3-6. The results were evaluated in comparison to corresponding reference silica, GT-Si and GF-Si, while VN3 results were not taken into account. In most 
3 SBR-Functionalized Silica Nanoparticles - Grafting-from versus Grafting-to Approach

cases the physical properties were improved by the functionalization of silica. While the rebound was enhanced significantly for all samples, the influence on stress strain behavior and Payne effect was only little. Considering the amount of grafted polymer, no clear trend was found, but regarding most physical properties it seems to have an impact. To investigate this further, it would be necessary to prepare compounds with the same crosslink distance, since network strength has a great impact on mechanical properties. This can be achieved by the adjustment of the vulcanization system.

Hardness RT, M100 RT and Payne effect are indicators for filler-filler interactions within the compound. All samples, except for GF-DTPT, showed none or only little enhancement compared to the reference silica. Compared to the use of silane, the silica surface seems to be poorly shielded by surface bound polymer, resulting in strong filler-filler interactions. The results of GF-DTPT are contradictory, since hardness RT and M100 RT were enhanced compared to the use of silane, but on the other hand the Payne effect was worse than that of unfunctionalized silica. Nevertheless, there seems to be a critical amount of grafted polymer necessary to reduce filler-filler interactions.

The influence of functionalization on polymer-filler interactions is reflected in M300 RT and abrasion. Even though the crosslink distance was twice the value of the reference silica, the results were either equal or even significantly improved by functionalization of silica. In contrast to silane, the grafted polymer chains are able to entangle with the matrix polymer. This leads to an increase of polymer-filler interactions and reinforcement of the compound. Within this study, the end groups of the surface bound polymer were modified as thioether. This group acts as protective group for the sulphur atom, so it is not available for network formation during vulcanization. Further studies should be conducted with other polymer end groups, like thioester. This group is able to protect the sulphur atom during mixing, but is cleaved off during vulcanization. The resulting additional network linkage could also cause reinforcement of the polymer-filler interactions.

One challenge of the development of tire materials is, that optimizing one property always leads to deterioration of another. As an influence of the amount of grafted polymer was observed, this might be used to customize compounds, e.g. regarding the braking behavior or the rolling resistance. To circumvent this predicament, further studies could also include the mixing of various types of functionalized silica nanoparticles. The comparison of the use of silane and functionalization of silica particles showed, that 
first reduces filler-filler interactions and latter reinforces polymer-filler interactions. So the use of both in combination needs to be investigated too.

Both silica nanoparticles used for grafting approaches are less reinforcing than VN3. Latter represents a typical silica used in tire compounds. As fumed silica have a high tendency to aggregate, functionalization via grafting-from approach is challenging under the given reaction conditions. ${ }^{[78]}$ Within this study it was shown, that the dispersibility of silica particles within the highpressure autoclave can be improved by the choice of RAFT agent and might be investigated further. On the other hand, the problem of particle dispersion can be circumvented using the grafting-to approach. But both synthetic strategies used only yield low amounts of grafted polymer. Therefore, other synthetic strategies for grafting-to approach should be applied or rather be developed. Overall it was shown, that the functionalization of silica is a powerful tool to adjust the mechanical properties of tire compounds. 



\section{Anchor Groups-Containing Copolymers Grafted to Carbon Black}

This chapter presents the functionalization of carbon black via a grafting-to approach with copolymers containing anchor groups within their polymer side chain. The fundamentals of this approach, the oxidation of the CB surface and the chosen anchor groups, are introduced. By using methacrylate copolymers, the suitability of the anchor groups and the grafting conditions are investigated. Since the very uncommon pentyl methacrylate (PnMA) monomer was used within parts of this work, the determination of the MarkHouwink coefficients (MHC) of the corresponding homopolymer (PPnMA) as well as the investigation of its radical polymerization kinetics are presented. Furthermore, the influence of the functionalization of CB for usage as filler in rubber compounds is studied. Therefore, the copolymerization of butadiene with anchor groups-containing monomers is investigated.

Sections 4.2 and 4.3 and Subsection 4.4 .4 were conducted in close cooperation with Laura Brinkmann as part of her bachelor thesis. ${ }^{[88]}$ The determination of Mark-Houwink coefficients of PPnMA (Subsection 4.4.1) and investigation of the radical polymerization kinetics of PnMA (Subsection 4.4.2) have already been published and the results were adapted with permission of the journal and the authors. 


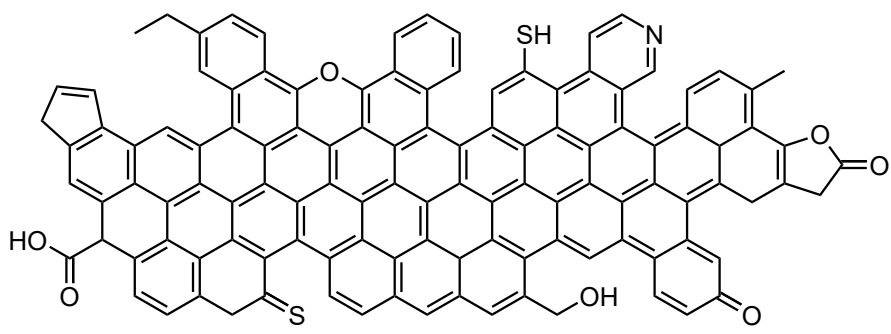

Figure 4-1: Carbon Black surface with functional groups located at defects within the polyaromatic structure.

\subsection{CB as Filler - Properties and State of the Art}

Carbon black is an amorphous material made of $90-99 \%$ elemental carbon in the form of polyaromatic structures. ${ }^{[00,91]}$ Layers of these structures build up primary particles, which accumulate due to strong van der Waals interactions. ${ }^{[92]}$ The size of the aggregates varies between 100 to $800 \mathrm{~nm}$ and they present the smallest dispersible units of $\mathrm{CB} .{ }^{[3]}$ The reactivity of $\mathrm{CB}$ is determined by functional groups at the surface, located at defects within the aromatic system (Figure 4-1). $00,91,93]$ The percentage of heteroatoms varies depending on the production process and can be increased e.g. by oxidation. $[91,94,95]$

A typical approach for the functionalization of $\mathrm{CB}$ as well as for related carbon nanotubes and graphene is the use of Diels-Alder reaction with

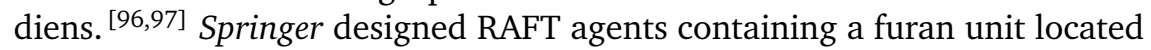
at the R group which could be bound to the CB surface prior to polymerization. [11] Another grafting-from approach was introduced by Wang et al. using Steglich esterfication to bind a RAFT agent in a multi-step reaction sequence. ${ }^{[98]}$ But since $\mathrm{CB}$ is a radical trap, the grafting-from approach is not as efficient as for other inorganic materials, like silica nanoparticles. [99] Using the grafting-to approach, this problem can be overcome. Some grafting-to approaches with polymers containing anchor groups within the polymer side chain were reported in literature. ${ }^{[100+102]}$ Besides the grafting via covalent bonds, e.g. by esterfication or amide bonds, also non-covalent functionalization with aromatic units like pyrene are reported. ${ }^{[103]}$ 


\subsection{Oxidation of CB Surface}

Depending on the reaction mechanism of functionalization a pretreatment of CB is necessary. This chemical modification is enabled by defects at the CB surface (Figure 4-1). ${ }^{[95]}$ An increase of the amount of oxygen-containing functional groups can be obtained via oxidation, plasma treatment or using oxidants. $98,104,105]$ The choice of oxidant determines which kind of functional groups are formed primarily.

The oxidation with nitric acid $\left(\mathrm{HNO}_{3}\right)$ to introduce carboxy groups (CB$\mathrm{COOH}$ ) was conducted according to Yang et al. (Scheme 4-1). ${ }^{[98]}$ The functionalization with hydroxy groups $(\mathrm{CB}-\mathrm{OH})$ was performed according to Beckert et al., using a modified Hummers method (Scheme 4-2). ${ }^{[105]}$ After oxidation, the particles were purified via centrifugation and dried in vacuo at $60^{\circ} \mathrm{C}$ for $24 \mathrm{~h}$.

The successful oxidation by both methods was confirmed by TGA (Figure 4-2) and EA (Table 4-1). The TGA curves of oxidized CB particles show a decrease of mass until $500{ }^{\circ} \mathrm{C}$ of around $16 \mathrm{wt} \%$ for $\mathrm{CB}-\mathrm{COOH}$ and $20 \mathrm{wt} \%$ for $\mathrm{CB}-\mathrm{OH}$ compared to the untreated $\mathrm{CB}$. Even though no distinguishable main weight loss regions can be identified, the weight loss can be assigned to adsorbed water and the decomposition of functional groups at the surface. ${ }^{[106]}$ Elemental analysis supports this conclusion by showing a decrease of carbon percentage and an increase of hydrogen amount after the oxidation. The percentage of oxygen could not have been measured directly, but can be estimated from the difference of carbon percentage before and after oxidation, resulting in an increase of around $14 \mathrm{wt} \%$ for $\mathrm{CB}-\mathrm{COOH}$ and $22 \mathrm{wt} \%$ for $\mathrm{CB}-\mathrm{OH}$.

Further analysis of the resulting functional groups was not conducted. Both synthetic strategies might also yield other functional groups than the targeted ones. Still, according to literature, it is assumed that most functional groups are carboxy or hydroxy groups. $[98,105]$

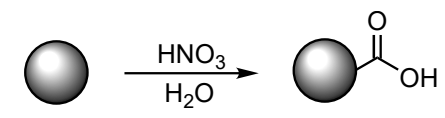

Scheme 4-1: Schematic representation of the functionalization of CB via oxidation with nitric acid. 


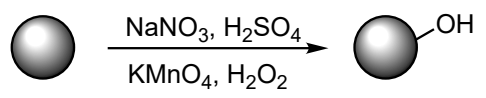

Scheme 4-2: Schematic representation of the oxidation of CB via Hummers method.

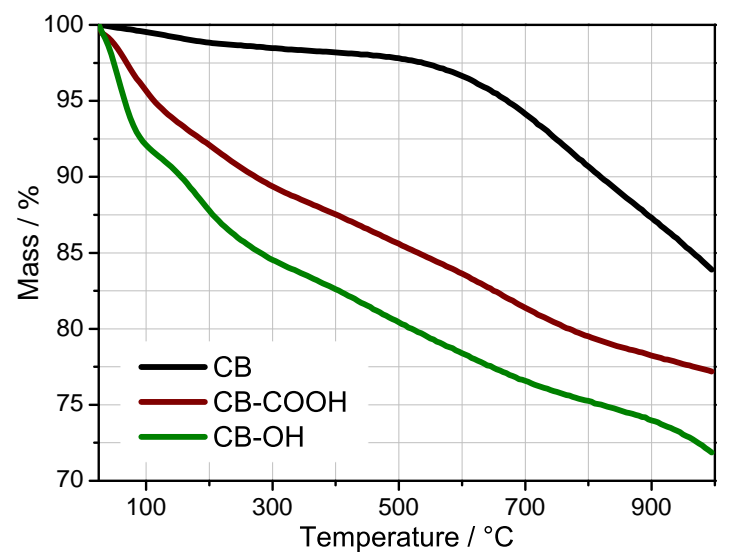

Figure 4-2: Thermograms of untreated and oxidized CB measured under $\mathrm{N}_{2}$ atmosphere.

Table 4-1: Weigth percentage of carbon, hydrogen, nitrogen, sulfur and oxygen before and after the oxidation measured by EA. *The amount of oxygen could not have been measured directly and was therefore estimated from the difference of carbon percentage before and after oxidation.

\begin{tabular}{lccccc}
\hline & $\mathrm{C}$ & $\mathrm{H}$ & $\mathrm{N}$ & $\mathrm{S}$ & $\mathrm{O}^{*}$ \\
\cline { 2 - 6 } & \multicolumn{5}{c}{$/ \mathrm{wt} \%$} \\
\hline CB & 96.33 & 0.26 & 0.17 & $<1$ & \\
CB-COOH & 82.12 & 0.84 & 0.72 & $<1$ & 14 \\
CB-OH & 74.07 & 1.64 & 0.30 & 1.84 & 22 \\
\hline
\end{tabular}




\subsection{Introduction of the Anchor Groups for Grafting to $\mathrm{CB}$}

The chemical structure of $\mathrm{CB}$ offers a variety of possible reactions that can be performed at the surface. ${ }^{[95]}$ With regards to the main goal of this work, the functionalization of nanoparticles with SBR for usage as filler in tire compound, some conditions for the choice of anchor groups had to be fulfilled. First, the corresponding monomers need to be suitable for butadiene copolymerization. Second, the grafting reaction should be simple and without the use of further chemicals, so that grafting could become possible during the mixing of the tire compound. Considering this, furan, epoxide and pyridine were chosen as anchor groups and the grafting mechanisms are shown in Scheme 4-3.

The furan unit is bound to $\mathrm{CB}$ via Diels-Alder reaction. The advantage of this anchor group is that no pretreatment of $\mathrm{CB}$ is necessary. ${ }^{[11]} \mathrm{A}$ disadvantage could be that the bond might not be stable during vulcanization of the rubber compound, since Retro-Diels-Alder reaction occurs at temperatures above $160^{\circ} \mathrm{C}$. [107]

Epoxidized natural rubber is already applied as a compatibilizer in rubber compounds. ${ }^{[01]}$ The epoxide unit is bound by a ring opening reaction to the CB surface. For this approach, an increase of hydroxyl groups at the surface could enhance the grafting density. ${ }^{[108]}$

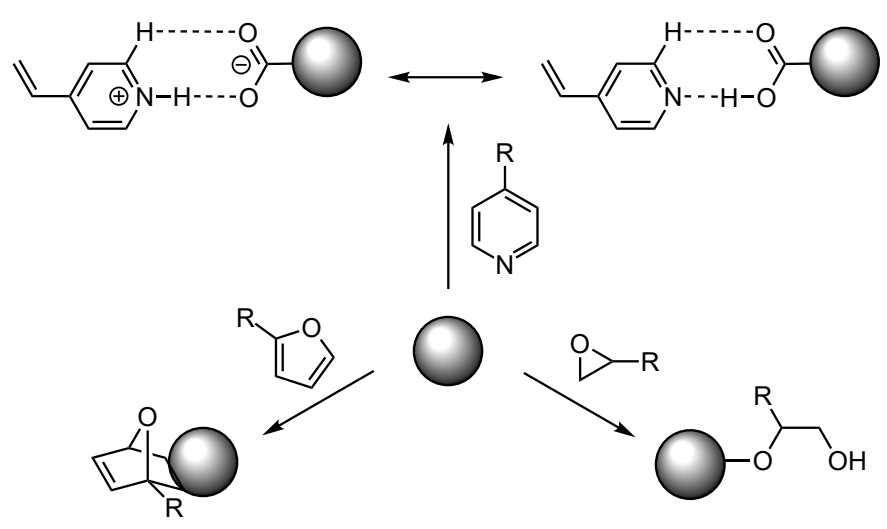

Scheme 4-3: Schematic representation of the grafting mechanisms of the furan, epoxide and pyridine units to $\mathrm{CB}$. 
The pyridine unit binds via non-covalent $\pi-\pi$ interactions and hydrogen bonds. ${ }^{[103,109]}$ The amount of the latter could be enhanced by increasing the amount of carboxyl groups at the surface. This non-covalent approach could be interesting regarding the self-healing ability of the hybrid material. [110]

\section{Copolymerization of the Anchor Groups-Containing Monomers}

The polymerization of butadiene copolymers within this work can only be realized using the high-pressure autoclave (Section 3.2). Since the experimental expense is high and large quantities of the substances are needed for compounding, another class of monomers was chosen as a model system for preliminary experiments. Acrylate and methacrylate polymers are often used for the investigation of mechanical properties of hybrid materials. Usually, the use of acrylic monomers would be favoured due to their much shorter reaction time. But it is known for radical polymerizations of acrylates that monomers with aromatic side groups can undergo undesired crosslinking or inhibit the polymerization. ${ }^{[111-113]}$ In contrast to that, the polymerization of methacrylates with aromatic side chains is uncritical. ${ }^{[113]}$ Another advantage is the commercial availability of the required monomers (Figure 4-3).

Poly(hexyl methacrylate) (PHMA) was chosen as the matrix polymer due to its glass transition temperature $\left(T_{\mathrm{g}}\right)$ around room temperature. ${ }^{[114]}$ Glycidyl methacrylate (GMA), furfuryl methacrylate (FMA) and 4-vinylpyridine i] (VP) were the corresponding anchor group-containing comonomers.

\footnotetext{
iVinylpyridine consists of two constitutional isomers: 2-vinylpyridine and 4-vinylpyridine. The 1,2-isomer does not have any importance within this work therefore vinylpyridine will be used synonymous for 1,4-vinylpyridine.
}

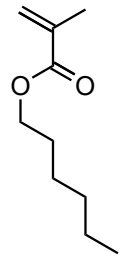

(a)

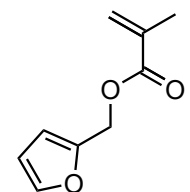

(b)

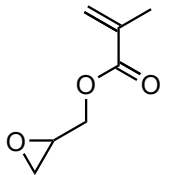

(c)<smiles>C=Cc1ccncc1</smiles>

(d)

Figure 4-3: Structural formulas of (a) HMA, (b) FMA, (c) GMA and (d) VP. 
Table 4-2: Number average molecular mass, dispersity and comonomer ratio of hexyl methacrylate copolymers after different polymerization periods. Polymerizations were performed with CDSPA and AIBN in $50 \mathrm{wt} \%$ toluene at $60^{\circ} \mathrm{C}$ and with a monomer-to-RAFT-to-initiator ratio of $500: 1: 0.2$ and $10 \mathrm{~mol} \%$ of the comonomer.

\begin{tabular}{lcccc}
\hline & $t / \mathrm{h}$ & $\bar{M}_{\mathrm{n}} / 10^{4} \mathrm{~g} \mathrm{~mol}^{-1}$ & $\oplus$ & $x_{\mathrm{Co}} / \mathrm{mol} \%$ \\
\hline \multirow{3}{*}{ P(HMA-co-VP) } & 6 & 4.5 & 1.2 & 15.9 \\
& 12 & 4.9 & 1.2 & 16.4 \\
& 15 & 5.1 & 1.2 & 16.9 \\
\hline \multirow{2}{*}{ P(HMA-co-FMA) } & 6 & 4.3 & 1.4 & 8.6 \\
& 12 & 8.1 & 1.6 & 7.9 \\
& 15 & 7.9 & 1.6 & 7.7 \\
P(HMA-co-GMA) & 6 & 5.1 & 1.2 & 11.5 \\
& 12 & 7.1 & 1.2 & 12.3 \\
& 15 & 7.5 & 1.2 & 12.2 \\
\hline
\end{tabular}

The copolymerizations of hexyl methacrylate (HMA) with $10 \mathrm{~mol} \%$ of the comonomers were conducted with CDSPA as RAFT agent at $60^{\circ} \mathrm{C}$ in $50 \mathrm{wt} \%$ toluene. Table 4-2 gives an overview of the number average molecular mass, the dispersity $(\nexists)$ and the comonomer ratio $\left(x_{\mathrm{Co}}\right)$ within the polymer after different periods of polymerization. SEC analysis was performed using the Mark-Houwink coefficients of HMA and the corresponding MDWs are shown in Figure E.1. [115] The comonomer ratio was determined via ${ }^{1} \mathrm{H}-\mathrm{NMR}$ analysis by comparison of the intensities of the protons in $\alpha$-position to the methacrylic group of HMA and the ring protons of the comonomers. The results of SEC and ${ }^{1} \mathrm{H}-\mathrm{NMR}$ analysis in Table 4-2 confirmed the successful copolymerizations and a good polymerization control by CDSPA, reflected in low dispersities. Copolymerizations with FMA and GMA seem to yield statistical copolymers, since the ratio before and after polymerization is approximately the same. In contrast to that, the ratio of VP in the copolymer is 1.5 times higher than in the monomer mixture. This indicates the formation of a gradient copolymer. 

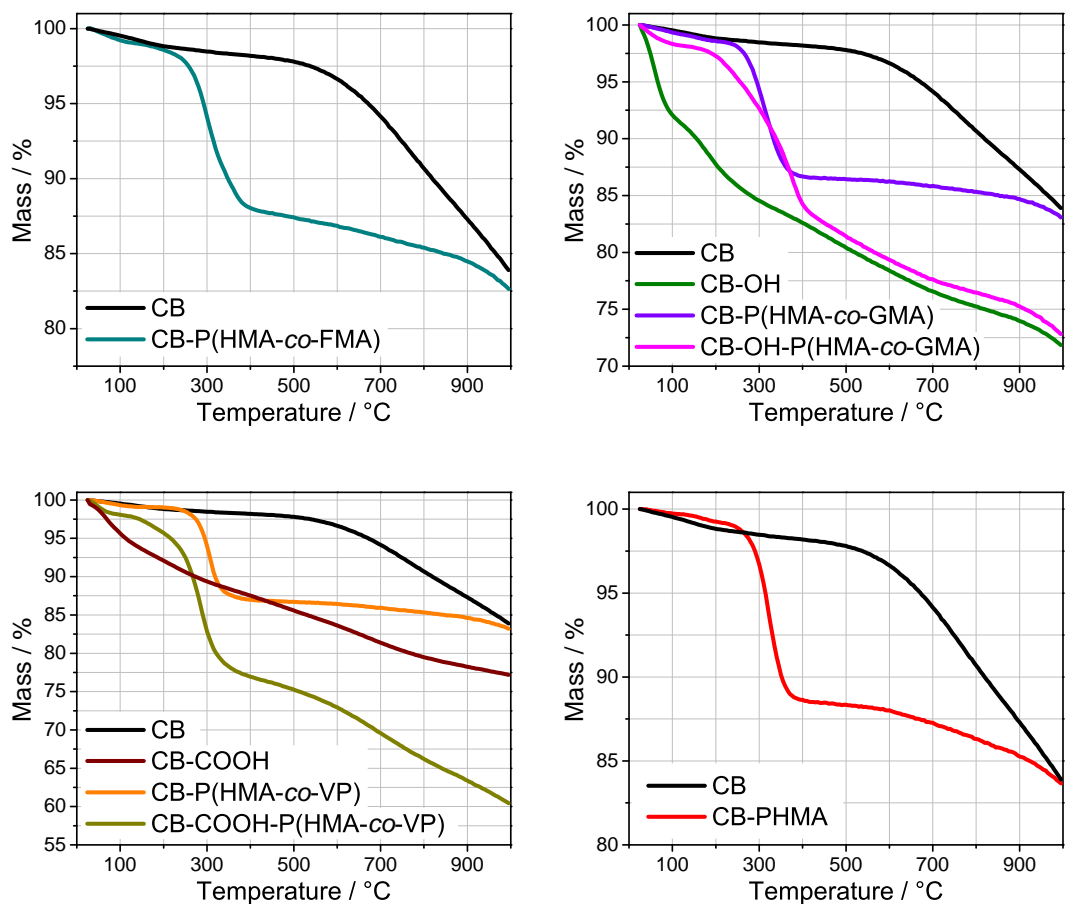

Figure 4-4: Thermograms of CB before and after the functionalization with hexyl methacrylate copolymers measured under $\mathrm{N}_{2}$ atmosphere.

\section{Grafting of Copolymers to CB}

The functionalization of CB was performed in dibenzyl ether (DBE). The copolymers were dissolved in triple amount of DBE, $20 \mathrm{wt} \% \mathrm{CB}$ were added and the dispersions were stirred for $2 \mathrm{~h}$ at $120^{\circ} \mathrm{C}$. The reaction temperature was chosen in accordance to the typical mixing temperature of rubber compounds. The particles were washed three times with tetrahydrofuran (THF) to get rid of free polymer and were dried in vacuo.

Figure 4-4 depicts the thermograms of the CB particles before and after (denoted by the type of CB particles used for the functionalization and the bound polymer) the functionalization under $\mathrm{N}_{2}$ atmosphere. The weight loss region from 200 to $450^{\circ} \mathrm{C}$ was assigned to the decomposition of the grafted 
Table 4-3: Ratio of the comonomers within the used copolymers and weight loss of $\mathrm{CB}$ particles before and after the functionalization determined by TGA under $\mathrm{N}_{2}$ atmosphere between $200-450^{\circ} \mathrm{C}$.

\begin{tabular}{lcc}
\hline & $x_{\mathrm{Co}} /$ mol\% & Weight loss $/ \mathrm{wt} \%$ \\
\hline CB & & 0.9 \\
CB-OH & & 6.2 \\
CB-COOH & 8.6 & 5.5 \\
CB-P(HMA-co-FMA) & 12.3 & 10.9 \\
CB-P(HMA-co-GMA) & 12.3 & 12.0 \\
CB-OH-P(HMA-co-GMA) & 16.4 & 14.7 \\
CB-P(HMA-co-VP) & 16.4 & 12.2 \\
CB-COOH-P(HMA-co-VP) & & 21.2 \\
CB-PHMA & & 10.6 \\
\hline
\end{tabular}

copolymers and the corresponding weight losses are given in Table 4-3. All functionalized CB samples show a weight loss in this area, which indicates a successful functionalization of $\mathrm{CB}$ by the anchor groups. Ranging from 10.0 to $12.2 \mathrm{wt} \%$, the weight losses for $\mathrm{CB}$ without pretreatment are relatively similar. In case of GMA and VP, the weight loss is increased through the usage of oxidized $\mathrm{CB}$. Within the respective temperature region the oxidized CB particles also exhibit a weight loss before the functionalization (Table 4 2). On the one hand this weight loss originates from the decomposition of the functional groups at the $\mathrm{CB}$ surface introduced via oxidation. On the other hand it might also come from adsorbed solvent and water molecules trapped within the CB layers or agglomerates. Through the encapsulation their decomposition temperature is increased. A similar behavior is known e.g. from silica nanoparticles prepared via Stöber method (Subsection 3.3.1). Since it is not known if these encapsulated solvent or water molecules are also present after the functionalization, the amount of grafted copolymer can only be estimated by the difference of the weight losses of the oxidized $\mathrm{CB}$ particles before and after the functionalization. For oxidized CB functionalized with GMA copolymer the estimated amount of grafted copolymer is lower compared to the use of untreated $\mathrm{CB}$, which is contradictory to the proposed binding mechanism in Scheme 4-3. But in case of VP the amount of grafted copolymer is increased through the use of oxidized CB. This might 
be an indicator that more copolymer is grafted to the CB surface through additional hydrogen bonds.

Additionally, a sample of pure PHMA was mixed with CB under the same grafting conditions as a reference. The weight loss of the resulting $\mathrm{CB}$ particles of $10.6 \mathrm{wt} \%$ is similar to that of the copolymers with untreated CB. This indicates that adsorption of polymer to CB occurs, since PHMA does not contain anchor groups for $\mathrm{CB}$. This is an important fact to be considered when it comes to the interpretation of the TGA curves.

\section{Solution Behavior of Functionalized CB}

Using CB, it is barley possible to distinguish between grafted or simply adsorbed polymer by analytical methods. Under the assumption that adsorbed polymer is bound weaker to the surface than grafted polymer, the solution behavior could provide insights into the binding situation. Non-functionalized $\mathrm{CB}$ is hardly dispersable in THF, which is a good solvent for the copolymers. Through functionalization of $\mathrm{CB}$ by grafting of polymer, the solubility of $\mathrm{CB}$ in THF is enhanced.

The same weight fractions of CB particles were dispersed in THF under sonication for $5 \mathrm{~min}$ and the precipitation of the particles was monitored for 7 days (Figure 4-5). Precipitation of untreated CB, CB-COOH and CB-PHMA was observed already after $5 \mathrm{~min}$. $\mathrm{CB}-\mathrm{OH}$ showed significant precipitation after 90 min. CB-P(HMA-co-GMA) started to settle down after $210 \mathrm{~min}$, followed by CB-P(HMA-co-FMA) after 24h. CB-OH-P(HMA-co-GMA) showed slight precipitation after 7 days, while the $\mathrm{CB}$ particles containing vinylpyridine still remained dispersed.

The fact that CB-HMA behaved like non-functionalized CB samples indicates, that the polymer is only bound weakly to the surface through adsorption. The functionalized $\mathrm{CB}$ particles started to settle down afterwards. From this results it can be concluded that at least a fraction of the copolymer is bound to CB through the anchor groups. As discussed before, in case of the GMA copolymer the amount of grafted polymer was lower using the oxidized CB compared to the untreated $\mathrm{CB}$. The results of the solution behavior showed, that the untreated $\mathrm{CB}$ particles functionalized with the GMA copolymer settled down much earlier compared to the oxidized particles. This indicates, that the fraction of covalently bound GMA copolymer is increased by the oxidation of the $\mathrm{CB}$ particles, supporting the proposed binding mechanism in Scheme 4-3. Both the untreated and oxidized CB particles functionalized with the VP copolymer still remained dispersed after 


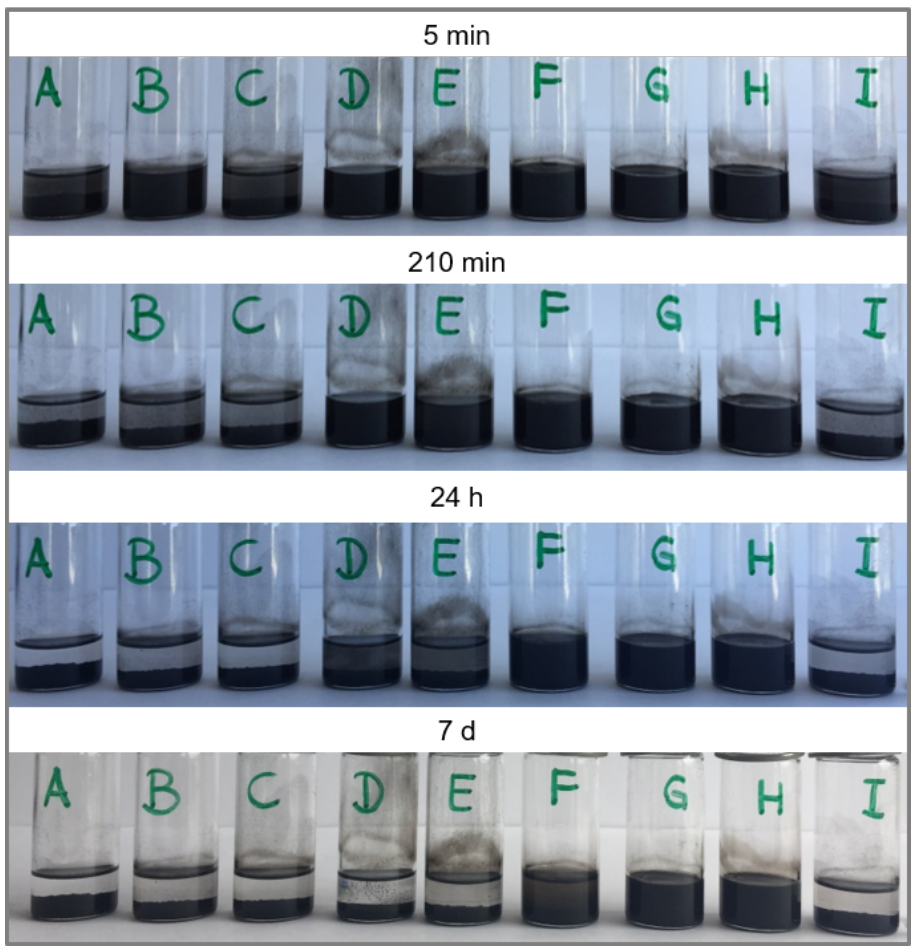

Figure 4-5: CB samples dispersed in THF under sonication and monitored afterwards for 7 days.

A: CB, B: CB-OH, C: CB-COOH, D: CB-P(HMA-co-FMA), E: CB-P(HMAco-GMA), F: CB-OH-P(HMA-co-GMA), G: CB-P(HMA-co-VP), H: CBCOOH-P(HMA-co-VP), I: CB-PHMA.

one week. This points out that the majority of the VP copolymer is bound to the CB surface through the anchor groups and that these non-covalent interactions are strong, making the pyridine anchor a promising candidate for the functionalization of $\mathrm{CB}$. 


\subsection{Grafting of Pentyl Methacrylate Copolymers to CB}

After the successful grafting of the copolymers to CB, the influence of functionalization on the mechanical properties of filled composites was supposed to be investigated by DMA. It turned out, that composite test specimen, consisting of functionalized CB in a matrix of PHMA, were too soft to be measured with DMA. The measurement of PHMA in a material pocket showed a $T_{\mathrm{g}}$ of about $3^{\circ} \mathrm{C}$ taken from the maximum of the loss modulus (Figure 4-6). Consequently, the polymer is already in the viscous state at ambient temperature and another matrix polymer had to be found. As the copolymerizations with methacrylates worked well and the corresponding anchor groups-containing monomers are commercially available, the class of methacrylates had to be maintained. For the fabrication and the testing of specimen with DMA, a $T_{\mathrm{g}}$ little below room temperature is very suitable.

There are some reports in literature about the relaxation behaviour of poly(n-alkyl methacrylates), showing an almost linear increase of $T_{\mathrm{g}}$ with decreasing number of $n .{ }^{[114,116]}$ Beiner et al. determined the $T_{\mathrm{g}}$ of poly(butyl methacrylate) to be about $30^{\circ} \mathrm{C}$ via rheometry. ${ }^{[116]}$ With the linear trend concerning the relaxation behavior PPnMA was a promising candidate. The

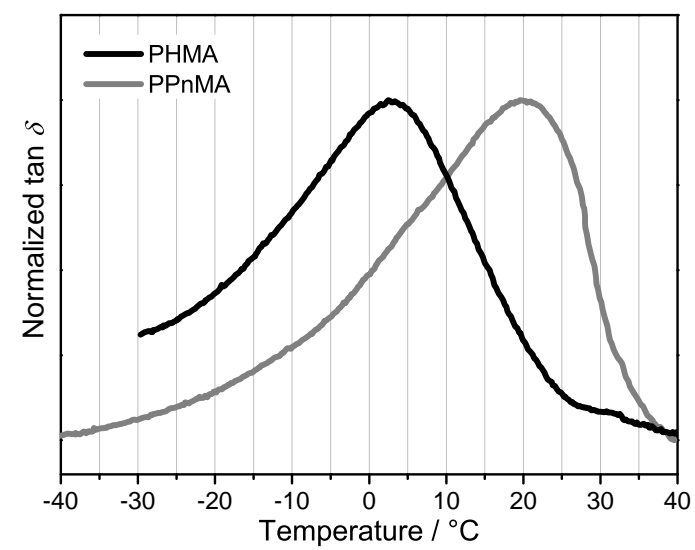

Figure 4-6: Temperature-dependent loss modulus of PHMA and PnMA measured by DMA in material pockets. 
$T_{\mathrm{g}}$ taken from the loss modulus of a DMA measurement in a material pocket was determined at $20^{\circ} \mathrm{C}$ (Figure 4-6). Thus, PPnMA is a suitable matrix polymer.

In contrast to other monomers of this class, the polymerization of pentyl methacrylate has not been studied in detail yet. Therefore, the kinetic parameters of the radical polymerization were determined via pulsed laser polymerization techniques. This required the determination of the temperaturedependent density $(\rho(T))$ of the monomer and of the Mark-Houwink coefficients of PPnMA for the analysis of the molar weight distribution via SEC. [89]

\subsubsection{Mark-Houwink Coefficients of PPnMA}

ii] For a given polymer, the Mark-Houwink equation (Equation (4.1)) relates intrinsic viscosity $([\eta])$ to molar mass $(M)$ :

$$
[\eta]=K \cdot M^{a}
$$

with $K$ and $a$ being the two Mark-Houwink parameter. [117,118] The SEC setup is mostly calibrated with poly(methyl methacrylate) (PMMA) or polystyrene standards and the molecular weight of a polymer under investigation is determined via universal calibration on the basis of the MarkHouwink coefficients of both polymers according to Equation (4.2).

$$
\log M_{1}=\frac{1+a_{2}}{1+a_{1}} \cdot \log M_{2}+\frac{1}{1+a_{1}} \cdot \log \frac{K_{2}}{K_{1}} .
$$

This approach was used in a reversed fashion. The parameters $K_{\mathrm{PPnMA}}$ and $a_{\text {PPnMA }}$, which were not known so far, have been deduced from a doublelog plot according to Equation (4.3), of the SEC data obtained with PMMA calibration in conjunction with calculated molecular weights of PPnMA samples from RAFT polymerizations. Equation (4.3) is a re-arranged form of Equation (4.2).

$$
\log \left(K_{\mathrm{PMMA}} \cdot M_{\mathrm{PMMA}}^{a_{\mathrm{PMMA}}+1}\right)=\log \left(K_{\mathrm{PPnMA}}\right)+\log M_{\mathrm{PPnMA}} \cdot\left(a_{\mathrm{PPnMA}}+1\right) .
$$

For the PPnMA samples from RAFT polymerization the theoretical numberaverage molecular weight has been estimated via Equation (4.4) from monomer

\footnotetext{
ii Adapted with permission from A. Nitschke, L. Riemann,L. Kollenbach,V. Braun,M. Buback,P. Vana, Macromolecular Chemistry and Physics 2020, 221 (1), 1900345.
} 
conversion $(U)$, from the molar mass of the monomer $\left(M_{\mathrm{M}}\right)$ and of the RAFT agent $\left(M_{\mathrm{RAFT}}\right)$ and from the initial concentrations of RAFT agent $\left(c_{\mathrm{RAFT}}\right)$ and of monomer $\left(c_{\mathrm{M}}^{0}\right) \cdot{ }^{[28] 30]}$

$$
\bar{M}_{\mathrm{n}, \text { theo }}=\frac{U \cdot c_{\mathrm{M}}^{0} \cdot M_{\mathrm{M}}}{c_{\mathrm{RAFT}}}+M_{\mathrm{RAFT}} .
$$

The Mark-Houwink Equation (4.1) is strictly speaking only valid for the viscosity average molar mass, $\bar{M}_{\mathrm{v}}$, as representative for molar mass $M$, which, however, is not accessible here. As an approximation, which is especially valid for narrowly dispersed polymer from RAFT polymerization, $\bar{M}_{\mathrm{n}}$ or the weigth average molar mass $\left(\bar{M}_{\mathrm{w}}\right)$ can substitute for $\bar{M}_{\mathrm{v}}$. As the exponent a is typically above 0.5 (also for PnMA as demonstrated below), $\bar{M}_{\mathrm{w}}$ is closer to $\bar{M}_{\mathrm{v}}$ and thus the better choice in order to minimize the error of the method. The weight-averaged molecular weight $\bar{M}_{\mathrm{w}, \mathrm{PPnMA}}$ is thus calculated via $\oslash$ using Equation (4.5), which is estimated from the SEC curve via PMMA calibration.

$$
\bar{M}_{\mathrm{w}, \mathrm{PPnMA}}=\bar{M}_{\mathrm{n}, \text { theo }} \cdot \bigoplus .
$$

Samples with molecular weights between 25000 and $111000 \mathrm{~g} \mathrm{~mol}^{-1}$ and with dispersities $D<1.3$ were synthesized under variation of both monomerto-RAFT ratio and polymerization time. The resulting double-log plot according to Equation (4.3) is shown in Figure 4-7. The Mark-Houwink coefficients of PPnMA at $35^{\circ} \mathrm{C}$ in THF were found from the linear fit to be: $K=25.7 \cdot 10^{-3} \mathrm{~mL} \mathrm{~g}^{-1}$ and $a=0.615$.

\subsubsection{Kinetics of the Radical Polymerization of PnMA}

iii For the design of polymerization processes the propagation rate coefficient $\left(k_{\mathrm{p}}\right)$ and the termination rate ${ }^{\mathrm{iv}}$ should be accurately known. ${ }^{[119-121]}$ The well-established method for $k_{\mathrm{p}}$ determination is pulsed laser polymerization in conjunction with size-exclusion chromatography (PLP-SEC). ${ }^{[120]}$ The $k_{\mathrm{p}}$ value is obtained from maxima $L_{\mathrm{i}}$ of the first-derivative curve of the MWD measured on the product from polymerization induced by a photoinitiator

\footnotetext{
iii Adapted with permission from A. Nitschke, L. Riemann,L. Kollenbach,V. Braun,M. Buback,P. Vana, Macromolecular Chemistry and Physics 2019, doi:10.1002/macp.201900345.

${ }^{\text {iv }}$ The termination kinetics of PnMA was investigated via single-pulse PLP electron resonance spectroscopy by Lara Riemann. As the results are presented within the respective publication, the results will not be discussed further within this work. 89$]$
} 


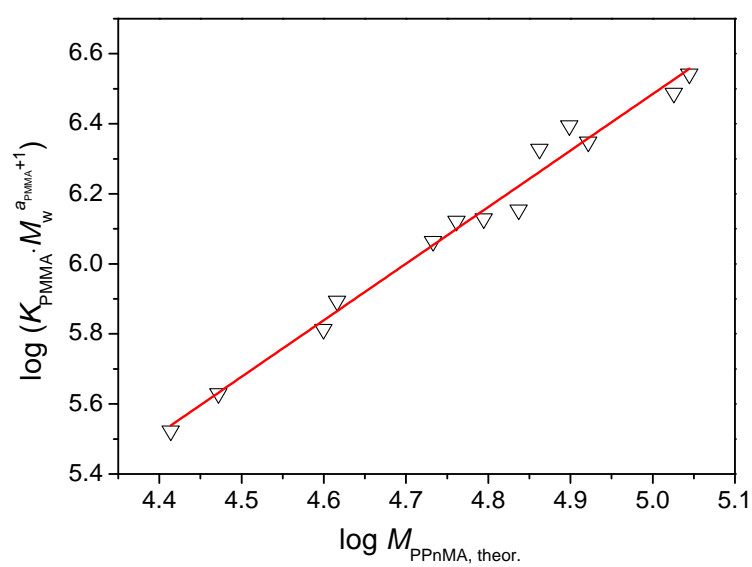

Figure 4-7: Double-log plot for determination of the Mark-Houwink coefficients of poly(pentyl methacrylate) according to Equation 4.3

under periodic laser pulsing at a precisely known laser pulse repetition rate, p.r.r.:

$$
L_{\mathrm{i}}=i \cdot k_{\mathrm{p}} \cdot c_{\mathrm{M}} \cdot t_{0} \quad i=1,2,3, \ldots
$$

where $c_{\mathrm{M}}$ is monomer concentration and $t_{0}$ is the time between two successive laser pulses, given by the inverse of pulse repetition rate. ${ }^{[122]}$ The first such maximum is mostly used for $k_{\mathrm{p}}$ determination. The reliability of the so-obtained $k_{\mathrm{p}}$ data should, however, be verified via the internal consistency criteria: [119]

$$
\frac{L_{1}}{L_{2}} \approx 0.50 \text { and } \frac{L_{2}}{L_{3}} \approx 0.67
$$

PLP-SEC analysis over a range of temperatures requires the knowledge of monomer density as a function of temperature. The measurements were performed at temperatures between 25.5 and $60.2{ }^{\circ} \mathrm{C}$ in bulk and in toluene solution. They resulted in linear relations of density vs. temperature for each concentration (Table 4-4). 
Table 4-4: Parameters of the linear fits of temperature-dependent density $\rho(T)=b-m \cdot T$ for PnMA in bulk and at different concentrations in toluene solution.

\begin{tabular}{lcc}
\hline$c_{\text {PnMA }} / \mathrm{M}$ & $m / 10^{-4} \mathrm{~g} \mathrm{KmL}^{-1}$ & $b / \mathrm{g} \mathrm{KmL}^{-1}$ \\
\hline 1.5 & 6.42781 & 1.0586 \\
2.7 & 6.39979 & 1.60122 \\
bulk & 6.20885 & 1.06314 \\
\hline
\end{tabular}
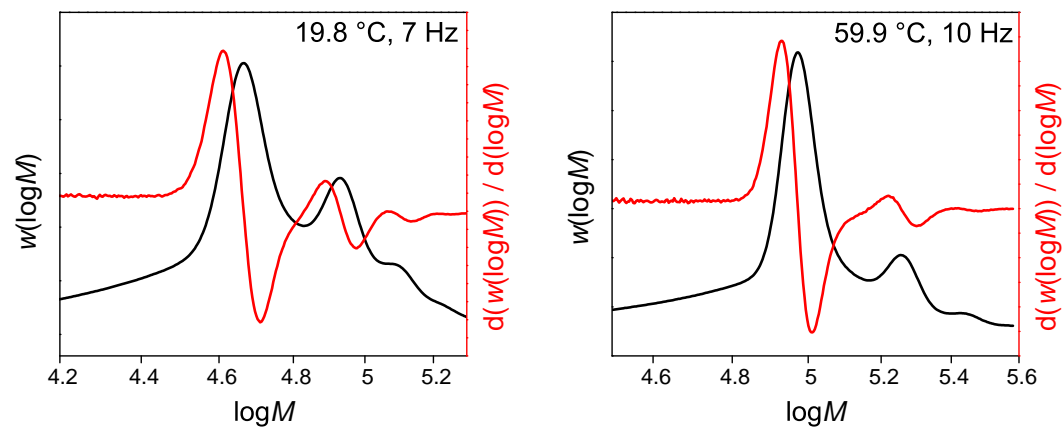

Figure 4-8: Molecular weight distribution and associated first-derivative curve for PLP-SEC experiments on bulk radical polymerizations of pentyl methacrylate at two temperatures and different p.r.r.'s. The abscissa refers to PMMA molar masses which have been used for calibration of the SEC setup.

\section{PLP-SEC Experiments in Bulk}

Pulsed laser polymerizations in bulk were performed between 19.8 and $59.9^{\circ} \mathrm{C}$. At each temperature, laser pulsing was carried out at p.r.r.'s of 7 and $10 \mathrm{~Hz}$. The number of applied laser pulses was chosen such that monomer conversion was kept below 5\%. Figure 4-8 shows the MWD's of PPnMA samples from PLP-SEC together with the associated first-derivative curves for two temperatures and different p.r.r.'s. The MWD's exhibit distinct first maxima, a smaller second maximum and a weak third maximum.

The $k_{\mathrm{p}}$ values were determined from the maxima of the first-derivate curves, in particular from the first such maximum, after calculation, via 
Table 4-5: Reaction conditions and results from the PLP-SEC analysis of the radical polymerization of pentyl methacrylate in bulk at ambient pressure with an initiator concentration of $c_{\text {DMPA }}=5.0 \mathrm{mmol} \mathrm{L}^{-1}$. Laser pulse repetition rate p.r.r., and the ratios of inflection points $L_{\mathrm{i}}$. Monomer conversion was always below $5 \%$.

\begin{tabular}{lcccc}
\hline$T /{ }^{\circ} \mathrm{C}$ & p.r.r. $/ \mathrm{Hz}$ & $k_{\mathrm{p}} / \mathrm{L} \mathrm{mol}^{-1} \mathrm{~s}^{-1}$ & $L_{1} / L_{2}$ & $L_{2} / L_{3}$ \\
\hline 21.0 & 7 & 354 & 0.51 & 0.66 \\
19.8 & 7 & 343 & 0.51 & 0.65 \\
22.4 & 10 & 357 & 0.50 & 0.68 \\
19.8 & 10 & 339 & 0.51 & 0.65 \\
36.5 & 7 & 594 & 0.50 & 0.64 \\
37.5 & 7 & 611 & 0.50 & \\
36.8 & 10 & 611 & 0.52 & 0.65 \\
37.7 & 10 & 604 & 0.51 & 0.65 \\
50.9 & 7 & 830 & 0.49 & \\
49.9 & 7 & 814 & 0.49 & \\
50.9 & 10 & 880 & 0.50 & 0.64 \\
49.9 & 10 & 864 & 0.50 & 0.65 \\
59.0 & 7 & 1055 & 0.46 & \\
57.9 & 7 & 1048 & 0.48 & \\
59.9 & 10 & 1091 & 0.50 & 0.63 \\
59.0 & 10 & 1136 & 0.49 & \\
\hline
\end{tabular}

Equation (4.2), the characteristic positions of PPnMA from the data referring to PMMA. The consistency criteria for reliable $k_{\mathrm{p}}$ analysis are fulfilled for the entire set of experiments, as testified by the listed ratios of inflection point positions. The bulk $k_{\mathrm{p}}$ values are summarized in Table 4-5. Depicted in Figure 4-9 is the temperature dependence of $k_{\mathrm{p}}$ for bulk PnMA. The Arrhenius fit represented by the straight line reads:

$$
\begin{aligned}
\ln \left[k_{\mathrm{p}}(\mathrm{PnMA}) /\left(\mathrm{L} \mathrm{mol}^{-1} \mathrm{~s}^{-1}\right)\right]= & 15.60-2861 \cdot(T / \mathrm{K})^{-1} \\
& \left(19.8^{\circ} \mathrm{C} \leq \theta \leq 59.9^{\circ} \mathrm{C}\right)
\end{aligned}
$$

with an activation energy of $E_{\mathrm{A}}\left(k_{\mathrm{p}}\right)=(23.8 \pm 0.4) \mathrm{kJ} \mathrm{mol}^{-1}$ and a preexponential factor of $A\left(k_{\mathrm{p}}\right)=(6.0 \pm 1.0) \cdot 10^{6} \mathrm{~L} \mathrm{~mol}^{-1} \mathrm{~s}^{-1}$. As can be seen from Table 4-6, both numbers are close to the ones of other alkyl methacry- 


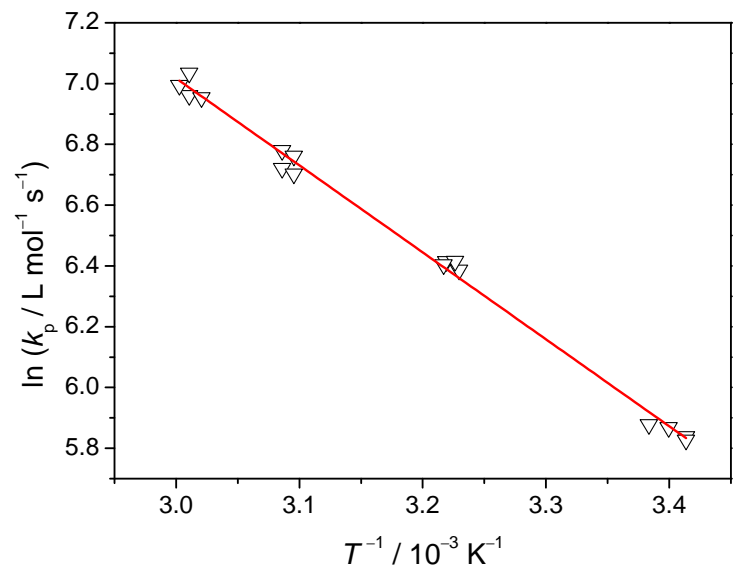

Figure 4-9: Temperature dependence of the propagation rate coefficient of bulk PnMA polymerization.

lates, which demonstrates the family behavior of methacrylate propagation rate. [122!123]

Benchmark values of $k_{\mathrm{p}}$ for several bulk alkyl methacrylates have been published as a function of polymerization temperature. This data from PLPSEC reveals an increase in $k_{\mathrm{p}}$ with increasing length of the alkyl side chain, e.g. in passing from MMA to dodecyl methacrylate (DMA), $k_{\mathrm{p}}$ increases by about $50 \% .{ }^{[122,123]}$ The Arrhenius straight lines for several alkyl methacrylates are plotted in Figure 4-10. Even within an experimental accuracy of $\pm 15 \%$, the data in Figure $4-10$ clearly indicates that bulk $k_{\mathrm{p}}$ increases toward larger size of the alkyl side chain. ${ }^{[122]}$ Moreover, the $k_{\mathrm{p}}$ of tert-BMA is slightly below the $k_{\mathrm{p}}$ of PnMA and also fits in this family behavior. [124]

\section{PLP-SEC Experiments in Toluene Solution}

PLP experiments on PnMA were also carried out in toluene solution, at two concentrations, p.r.r.'s of 7 and $10 \mathrm{~Hz}$ with monomer conversion being kept below $5 \%$. Figure 4-11 depicts normalized MWD's for samples from PLP-SEC experiments in toluene solution and in bulk at $50{ }^{\circ} \mathrm{C}$ and a p.r.r. of $10 \mathrm{~Hz}$. The MWD's exhibit a distinct first maximum, a smaller second 
Table 4-6: Arrhenius parameters for MMA, EMA, BMA, PnMA and DMA bulk radical polymerizations determined via PLP-SEC at ambient pressure.

\begin{tabular}{lccc}
\hline & $A\left(k_{\mathrm{p}}\right) / 10^{6} \mathrm{~L} \mathrm{~mol}^{-1} \mathrm{~s}^{-1}$ & $E_{\mathrm{A}}\left(k_{\mathrm{p}}\right) / \mathrm{kJ} \mathrm{mol}^{-1}$ & Ref. \\
\hline MMA & 2.7 & 22.4 & {$[123]$} \\
EMA & 4.1 & 23.4 & {$[122]$} \\
BMA & 3.8 & 22.9 & {$[122]$} \\
PnMA & 6.0 & 23.8 & this work \\
DMA & 2.5 & 21.0 & {$[122]$} \\
\hline
\end{tabular}

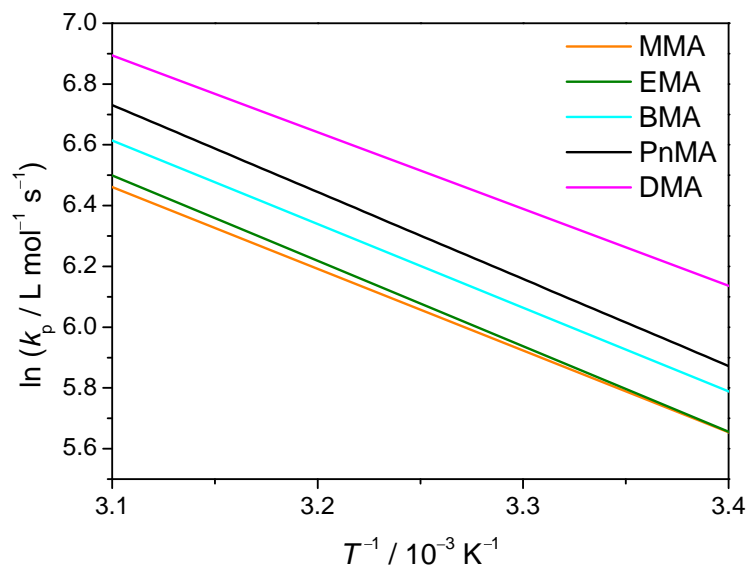

Figure 4-10: Arrhenius plot of the propagation rate coefficient for bulk radical polymerizations of methyl methacrylate (MMA), [123] ethyl methacrylate (EMA), ${ }^{[122]}$ butyl methacrylate (BMA), ${ }^{[122]}$ pentyl methacrylate (PnMA), and dodecyl methacrylate (DMA) [122].

maximum and even a weak third maximum. In passing from dilute solution $(1.5 \mathrm{M})$ to the bulk system, the maximum positions are shifted toward higher values because of the increasing monomer content. The $k_{\mathrm{p}}$ data for 1.5 and 2.7 molar solutions of PnMA in toluene are listed in Table 4-7. The ratios of inflection point positions again show that the consistency criteria are fulfilled. 


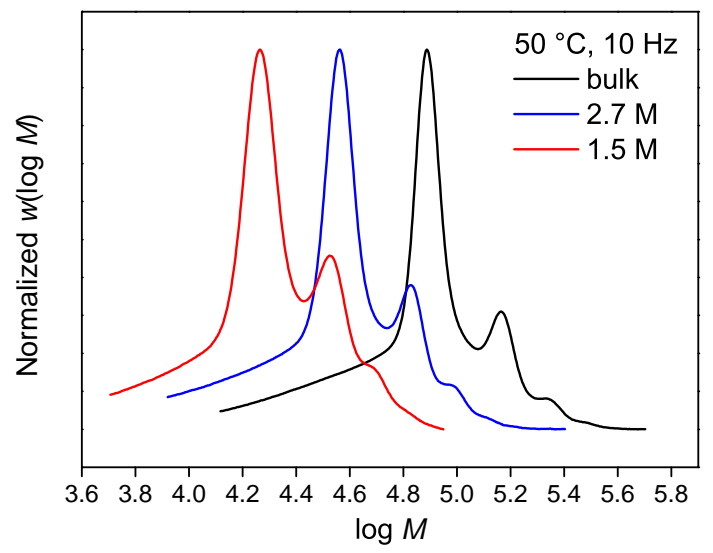

Figure 4-11: Normalized molecular weight distributions of PLP-SEC samples produced by radical polymerization of PnMA in bulk and in mixtures with toluene at $50^{\circ} \mathrm{C}$ at a p.r.r. of $10 \mathrm{~Hz}$. The SEC setup was calibrated with PMMA standards.

The temperature dependence of $k_{\mathrm{p}}$ for 1.5 and 2.7 M PnMA polymerizations in toluene solution is shown in Figure 4-12 together with the bulk $k_{\mathrm{p}}$ data. The straight-line fits to the $1.5 \mathrm{M}$ and to the bulk data reveal that $k_{\mathrm{p}}$ of PnMA decreases upon dilution in toluene. With the exception of MMA, no $k_{\mathrm{p}}$ data for other alkyl methacrylates in toluene solution have been reported. Beuermann et al. showed that the $k_{\mathrm{p}}$ for MMA in toluene solution is above the bulk MMA value. In contrast, the $k_{\mathrm{p}}$ values of iso-bornyl methacrylate in toluene solution are below bulk $k_{\mathrm{p}}$. ${ }^{[125]}$ The authors attributed these opposite effects on $k_{\mathrm{p}}$ to the difference of molar volume of monomer and solvent. [125]

Within studies into alkyl acrylate polymerizations in toluene solution, the group of Vairon made similar observations. ${ }^{[126]}$ With small acrylates, such as methyl acrylate, bulk $k_{\mathrm{p}}$ was lower than the solution value, whereas the opposite type of behavior, i.e., higher bulk $k_{\mathrm{p}}$, was found for acrylates with a large side chain, e.g., for nonyl acrylate. Despite some early debate on this finding, the adequate explanation of this effect is related to the impact of the molecular environment on the rotational mobility of the transition state for propagation. $\stackrel{[127-129]}{ }$ Larger such hindrance to rotational mobility 


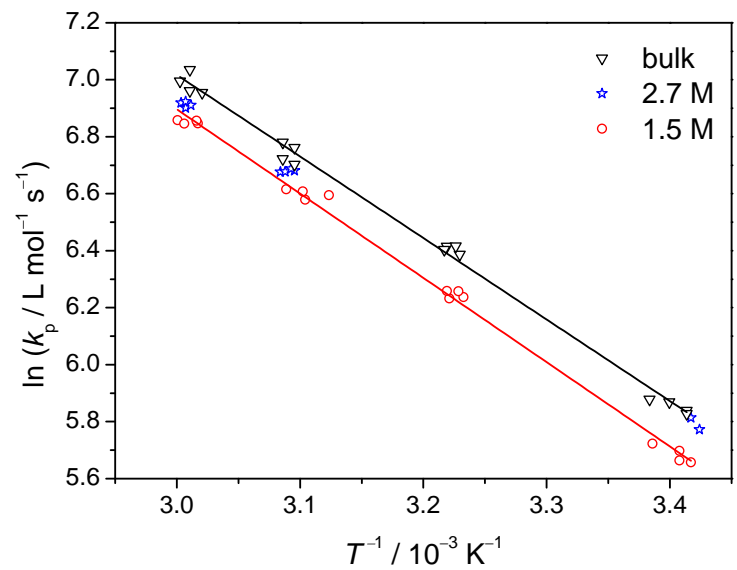

Figure 4-12: Temperature dependence of $k_{\mathrm{p}}$ for PnMA polymerizations in bulk and in toluene at different monomer concentrations.

in the transition state structure is accompanied by an entropy penalty which reduces the pre-exponential and thus lowers $k_{\mathrm{p}}$. The above-mentioned argument about the relative size of monomer and solvent molecules may be considered as a special case of the general entropy argument. As bulk $k_{\mathrm{p}}$ is lower than the value in toluene with small alkyl side chains, but is larger for long side chains, the trend must be reversed at intermediate sizes of the alkyl group. This is the case at about the alkyl size of butyl acrylate. [128]

The results for PnMA and for MMA suggest that the same type of behavior will probably occur within the methacrylate family. This assumption remains to be investigated by solution experiments, e.g. into dodecyl methacrylate in toluene. 
Table 4-7: Reaction conditions and results from the PLP-SEC analysis of the radical polymerization of pentyl methacrylate in toluene solution at ambient pressure with an initiator concentration of $c_{\mathrm{DMPA}}=$ $5.0 \mathrm{mmol} \mathrm{L}^{-1}$. Listed are the concentration of the initial monomer solution at room temperature $(c)$, the laser pulse repetition rate p.r.r., and the ratios of inflection points $L_{\mathrm{i}}$. Monomer conversion was always below $5 \%$.

\begin{tabular}{cccccc}
\hline$c / \mathrm{M}$ & $T /{ }^{\circ} \mathrm{C}$ & p.r.r. $/ \mathrm{Hz}$ & $k_{\mathrm{p}} / \mathrm{L} \mathrm{mol}^{-1} \mathrm{~s}^{-1}$ & $L_{1} / L_{2}$ & $L_{2} / L_{3}$ \\
\hline & 22.2 & 7 & 306 & 0.50 & 0.64 \\
& 20.3 & 7 & 298 & 0.50 & 0.64 \\
& 20.3 & 10 & 288 & 0.49 & 0.64 \\
19.5 & 10 & 286 & 0.49 & 0.64 \\
& 36.2 & 7 & 512 & 0.51 & 0.65 \\
& 37.3 & 7 & 509 & 0.50 & 0.65 \\
& 36.6 & 10 & 522 & 0.50 & 0.65 \\
1.5 & 37.5 & 10 & 522 & 0.52 & 0.64 \\
& 49.0 & 7 & 720 & 0.51 & 0.66 \\
& 50.6 & 7 & 747 & 0.51 & 0.65 \\
& 49.2 & 10 & 741 & 0.51 & 0.64 \\
& 47.0 & 10 & 731 & 0.51 & 0.63 \\
& 60.1 & 7 & 951 & 0.52 & \\
& 58.3 & 7 & 941 & 0.52 & 0.65 \\
& 59.5 & 10 & 940 & 0.51 & \\
& 58.4 & 10 & 949 & 0.51 & \\
\hline & 19.5 & 10 & 335 & 0.50 & 0.65 \\
& 18.9 & 10 & 321 & 0.52 & 0.64 \\
& 51.1 & 7 & 793 & 0.51 & 0.66 \\
& 49.9 & 7 & 797 & 0.51 & 0.67 \\
& 50.7 & 10 & 794 & 0.51 & 0.66 \\
& 50.2 & 10 & 802 & 0.50 & 0.66 \\
& 59.4 & 7 & 994 & 0.50 & \\
& 58.9 & 7 & 1003 & 0.51 & 0.65 \\
& 59.8 & 10 & 1011 & 0.51 & 0.67 \\
& 59.4 & 10 & 1016 & 0.51 & 0.66 \\
\hline \multirow{4}{*}{3.7} & & & &
\end{tabular}




\subsubsection{Investigation of the Grafting Conditions}

In the following section, the conditions for grafting of anchor groupscontaining copolymers to $\mathrm{CB}$ are further investigated to gain deeper understanding of the grafting mechanisms and the tuning of the grafting density. Untreated and oxidized CB particles were functionalized with PnMA copolymers (Table 4-8) under variation of time and temperature.

\section{Furan Anchor}

The grafting conditions and the weight loss after functionalization obtained from TGA are given in Table 4-9. The weight loss in TGA of untreated CB functionalized with FMA copolymer is equal under all conditions. This was unexpected, since it is known, that the mechanism of Diels-Alder reaction is temperature-dependent. Springer showed, that the grafting density of a furan-containing RAFT agent could be enhanced with increasing temperature up to $130^{\circ} \mathrm{C}$. [11] For both types of oxidized $\mathrm{CB}$ no weight loss was observable so the grafting of FMA copolymer to oxidized CB was not successful.

The investigation of the solution behavior of functionalized and nonfunctionalized CB in Section 4.3 showed, that the CB particles functionalized with FMA copolymer started to settle down much earlier than the other copolymer-functionalized particles. Keeping this in mind, there are two possible explanations for the given results. On the one hand, because of the high aromaticity of $\mathrm{CB}$, little percentage of furan units can bind to the surface

Table 4-8: Number average molecular mass, dispersity and comonomer ratio of pentyl methacrylate copolymers after different polymerization periods. SEC analysis was conducted using the Mark-Houwink coefficients of PPnMA. The MWDs of the copolymers are shown in Figure F.1. Polymerizations were performed with CDSPA and AIBN in bulk at $60^{\circ} \mathrm{C}$, a monomer-to-RAFT-to-initiator ratio of 250:1:0.2 was used. The comonomer ratio was $10 \mathrm{~mol} \%$ in case of FMA and GMA and $5 \mathrm{~mol} \%$ in case of VP.

\begin{tabular}{lcccc}
\hline & $t / \mathrm{h}$ & $\bar{M}_{\mathrm{n}} / 10^{4} \mathrm{~g} \mathrm{~mol}^{-1}$ & $Ð$ & $x_{\text {Co }} / \mathrm{mol} \%$ \\
\hline P(PnMA-co-VP) & 22 & 3.4 & 1.5 & 6.5 \\
P(PnMA-co-FMA) & 8 & 2.4 & 1.2 & 11.3 \\
P(PnMA-co-GMA) & 8 & 4.6 & 2.7 & 12.2 \\
\hline
\end{tabular}


Table 4-9: Weight loss of functionalized CB after the grafting of P(PnMAco-X) determined by TGA under $\mathrm{N}_{2}$ atmosphere between $200-450{ }^{\circ} \mathrm{C}$. The functionalization was conducted in DBE with $20 \mathrm{wt} \% \mathrm{CB}$ with respect to the copolymer.

\begin{tabular}{|c|c|c|c|c|c|}
\hline \multirow[b]{2}{*}{$\mathrm{X}$} & \multirow[b]{2}{*}{ CB } & \multicolumn{4}{|c|}{ Weight loss / wt $\%$} \\
\hline & & $\begin{array}{c}20^{\circ} \mathrm{C} \\
1 \mathrm{~h}\end{array}$ & $\begin{array}{c}20^{\circ} \mathrm{C} \\
18 \mathrm{~h}\end{array}$ & $\begin{array}{c}60^{\circ} \mathrm{C} \\
4 \mathrm{~h}\end{array}$ & $\begin{array}{c}120^{\circ} \mathrm{C} \\
2 \mathrm{~h}\end{array}$ \\
\hline \multirow{3}{*}{ FMA } & $\mathrm{CB}$ & 8 & 8 & 8 & 7 \\
\hline & $\mathrm{CB}-\mathrm{OH}$ & - & - & - & 0 \\
\hline & $\mathrm{CB}-\mathrm{COOH}$ & - & - & - & 0 \\
\hline \multirow{3}{*}{ GMA } & $\mathrm{CB}$ & 26 & 25 & 23 & 20 \\
\hline & $\mathrm{CB}-\mathrm{OH}$ & 25 & 26 & 19 & 36 \\
\hline & $\mathrm{CB}-\mathrm{COOH}$ & 31 & - & - & 35 \\
\hline \multirow{3}{*}{ VP } & $\mathrm{CB}$ & 9 & 9 & 9 & 9 \\
\hline & $\mathrm{CB}-\mathrm{OH}$ & 15 & 21 & - & 21 \\
\hline & $\mathrm{CB}-\mathrm{COOH}$ & 16 & 16 & 17 & 15 \\
\hline
\end{tabular}

even at low temperatures, but with increasing degree of functionalization or by oxidation Diels-Alder reaction can not proceed anymore, since the aromatic system is disturbed. On the other hand, the polymer is just adsorbed on the surface and by increasing the amount of functional groups through oxidation the van der Waals interactions between polymer and CB decrease, leading to less polymer on the surface. The preparation of reference samples of oxidized CB and PnMA homopolymer would be necessary to validate these theories.

\section{Epoxide Anchor}

The CB particles functionalized with the GMA copolymer show overall the highest weight loss compared to the other copolymers. With respect to the molecular mass of the copolymers (Table 4-8), the grafting density is four times that of the $\mathrm{CB}$ particles functionalized with the VP copolymer.

Before the discussion of the results it has to be noted, that the samples became poorly dispersable after functionalization, suggesting some kind of network formation. This behaviour was not observable under the use of the 
corresponding HMA copolymer. The MWD exhibits a shoulder (FigureF.1) and the resulting dispersity of the copolymer is 2.7 , which indicates network formation within the copolymer. But after polymerization or stirring in DBE at $120^{\circ} \mathrm{C}$, the polymer was still well soluble. Maybe, additional network formation is somehow catalyzed by CB during the grafting reaction.

The weight loss of GMA copolymer grafted to untreated CB is more or less independent of the conditions (Table 4-9), as been seen before for the particles functionalized with FMA copolymer. Compared to the samples with untreated $\mathrm{CB}$, the weight loss under the use of $\mathrm{CB}-\mathrm{OH}$ is only increased after reaction at $120^{\circ} \mathrm{C}$. This is in accordance with the results of the HMA copolymer, but the increase is significantly larger. The grafting to $\mathrm{CB}-\mathrm{COOH}$ at $120^{\circ} \mathrm{C}$ results in the same weight loss than for $\mathrm{CB}-\mathrm{OH}$. Furthermore, the highest weight loss after $1 \mathrm{~h}$ at $20^{\circ} \mathrm{C}$ was obtained for $\mathrm{CB}-\mathrm{COOH}$. The ratio of functional groups after the oxidation of $\mathrm{CB}$ could not be determined. As stated in literature, it was simply assumed that via Hummer's method mainly hydroxy functions and by use of nitric acid carboxy functions were introduced. ${ }^{[98,105]}$ The results of functionalization of both types of oxidized CB particles with the GMA copolymer indicate, that the amount of introduced hydroxy groups might be approximately the same using both oxidation methods.

Due to the network formation, the actual amount of copolymer grafted directly to the surface of $\mathrm{CB}$ can not be determined. Besides, the samples are unsuitable as filler for composites, since the dispersion within the matrix most propably be poor. A lower ratio of GMA within the copolymer might reduce the network formation.

\section{Pyridine Anchor}

The weight loss of grafted VP copolymer varied for the different types of CB but was more or less independent of the reaction conditions (Table 4-9]. As discussed before, oxidation increases the amount of hydrogen bonds, resulting in an enhanced grafting density. The results show, that the noncovalent grafting of pyridine anchors happens fast and without the need of any additional activation, since no chemical bond has to be formed.

In addition, copolymers with varying ratio of VP were synthesized and the weight loss after grafting to $\mathrm{CB}$ and $\mathrm{CB}-\mathrm{COOH}$ at room temperature was determined by TGA (Table 4-10). The results are comparable with the ones already discussed under similar conditions (Table 4-9). The weight loss of functionalized $\mathrm{CB}-\mathrm{COOH}$ particles is twice that of untreated $\mathrm{CB}$ particles. 
Table 4-10: Number average molecular mass, dispersity and comonomer ratio of P(PnMA-co-VP) and weight loss of copolymer after grafting to CB. SEC analysis was conducted using the Mark-Houwink coefficients of PPnMA (Subsection 4.4.1). Weight loss was determined by TGA under $\mathrm{N}_{2}$ atmosphere between $200-450{ }^{\circ} \mathrm{C}$. Polymerizations were performed with CDSPA and AIBN in bulk at $60^{\circ} \mathrm{C}$ for $22 \mathrm{~h}$ with a monomer-to-RAFT-to-initiator ratio of 250:1:0.2 with varying monomer ratios of VP $\left(x_{\mathrm{VP}, \mathrm{M}}\right)$. Functionalization was conducted at ambient temperature for $16 \mathrm{~h}$ in acetone with $66 \mathrm{wt} \% \mathrm{CB}$ with respect to the copolymer.

\begin{tabular}{lccccc}
\hline$x_{\mathrm{VP}, \mathrm{M}}$ & $\bar{M}_{\mathrm{n}}$ & $Ð$ & $x_{\mathrm{Co}}$ & \multicolumn{2}{c}{ Weight loss $/ \mathrm{wt} \%$} \\
\cline { 5 - 6 }$/ \mathrm{mol} \%$ & $/ 10^{4} \mathrm{~g} \mathrm{~mol}^{-1}$ & & $/ \mathrm{mol} \%$ & $\mathrm{CB}$ & $\mathrm{CB}-\mathrm{COOH}$ \\
\hline 12.5 & 3.0 & 1.4 & 10 & 14.7 & 21.2 \\
25 & 3.0 & 1.4 & 24 & 11.9 & 19.8 \\
50 & 2.8 & 1.2 & 38 & 11.2 & 19.8 \\
\hline
\end{tabular}

Regarding the comonomer ratio no significant difference of the weight loss can be observed. Since the molar masses of copolymers are alike, this indicates that the amount of grafted polymer is independent of the ratio of VP within the copolymer.

The properties of the non-covalent grafting mechanism make the pyridine anchor the most promising anchor group. It might be used to design selfhealing materials containing CB. Furthermore, functionalization of CB does not necessarily need to be performed prior to mixing of a compound, since the reaction is fast and might be done in situ.

\subsubsection{Dynamic Mechanical Analysis of CB-P(PnMA-co-X) Filled Composites}

The influence of functionalization of $\mathrm{CB}$ on its filler properties was investigated via DMA. Test specimen were produced by injection moulding. Therefore matrix polymer was dissolved in acetone, CB was added and the dispersion was mixed under sonication for $20 \mathrm{~min}$. Before injection moulding, the composites and the pure matrix polymer were dried in vacuo at $80^{\circ} \mathrm{C}$ for 5 days. The composites consisted of PPnMA as matrix with $10 \mathrm{wt} \%$ of CB (Table 4-11). The amount of CB of functionalized particles was calculated with respect to the residual mass at $450{ }^{\circ} \mathrm{C}$ determined by TGA. The temperature- 
Table 4-11: Number average molecular mass, dispersity and comonomer ratio of PPnMA and pentyl methacrylate copolymers and weight loss of copolymers after grafting-to CB. SEC analysis was conducted using the Mark-Houwink coefficients of PPnMA (Subsection 4.4.1). Weight loss was determined by TGA under $\mathrm{N}_{2}$ atmosphere between $200-450^{\circ} \mathrm{C}$. Polymerizations were performed with CDSPA and AIBN in $50 \mathrm{~V} \%$ toluene at $60^{\circ} \mathrm{C}$ for $22 \mathrm{~h}$ (VP comonomer) or $8 \mathrm{~h}$ (other samples) with a monomer-to-RAFT-to-initiator ratio of 250:1:0.2. The comonomer ratio was $10 \mathrm{~mol} \%$ in case of FMA and GMA and $5 \mathrm{~mol} \%$ in case of VP. Functionalization was conducted at $120^{\circ} \mathrm{C}$ for $2 \mathrm{~h}$ in DBE with 20 wt $\%$ CB with respect to the copolymer.

\begin{tabular}{lcccc}
\hline & $\begin{array}{c}\bar{M}_{\mathrm{n}} \\
/ 10^{4} \mathrm{~g} \mathrm{~mol}^{-1}\end{array}$ & $Ð$ & $\begin{array}{c}x_{\mathrm{Co}} \\
/ \mathrm{mol} \%\end{array}$ & $\begin{array}{c}\text { Weight loss } \\
/ \mathrm{wt} \%\end{array}$ \\
\hline PPnMA & 2.9 & 1.2 & - & - \\
CB-P(PnMA-co-VP) & 3.1 & 1.2 & 7.5 & 11.5 \\
CB-P(PnMA-co-FMA) & 2.4 & 1.4 & 8.0 & 9.8 \\
CB-P(PnMA-co-GMA) & 2.5 & 1.2 & 12.9 & 9.6 \\
\hline
\end{tabular}

dependent storage modulus (Figure 4-13), loss modulus (Figure 4-14) and $\tan \delta$ (Figure 4-15) were determined between -60 and $80^{\circ} \mathrm{C}$ with a heating rate of $2 \mathrm{~K} \mathrm{~min}^{-1}$ in a single cantilever geometry. For each composite, three specimen were measured and the results were averaged.

The CB-containing samples show an enhanced storage modulus over temperature compared to the pure matrix polymer (Figure 4-13). Thereby the difference is big in the glassy state and low in the viscous state. This is in accordance to theory (Subsection 2.3.2), since the elastic part is increased by the addition of filler. [57] Samples filled with FMA and VP copolymerfunctionalized CB have the highest moduli, whereas no difference is observable for the GMA-containing sample compared to non-functionalized CB.

A similar trend can be seen for the loss modulus (Figure 4-14). The CB-containing samples show a significant increase of the loss modulus compared to the pure matrix polymer but the $T_{\mathrm{g}}$, which is presented by the maximum of the curves, is not shifted. This also correlates with the theory of filler properties (Subsection(2.3.2). Polymer-filler interactions enhance the viscosity, resulting in an increase of energy demand for the deformation of the composite. [57] Again, the samples filled with FMA and VP copolymer-functionalized CB show the highest moduli. 


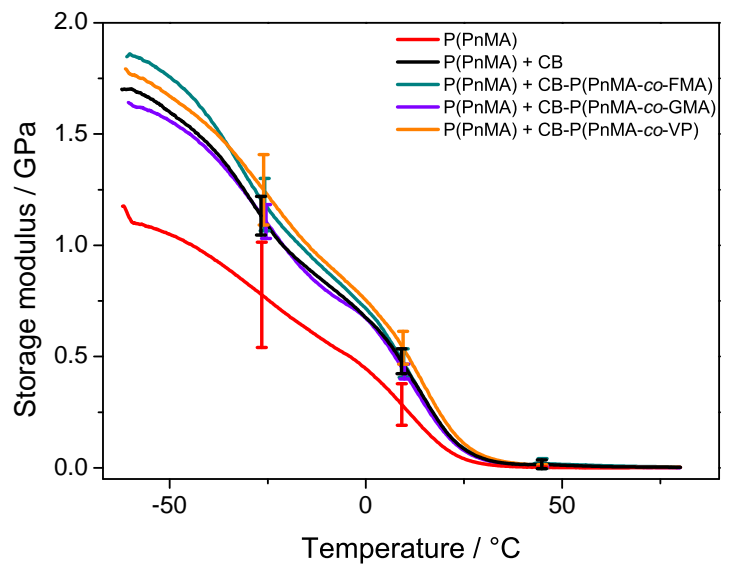

Figure 4-13: Temperature-dependent storage modulus of the pure matrix polymer PPnMA and of PPnMA composites filled with $10 \mathrm{wt} \%$ of untreated and functionalized CB.

The $\tan \delta$ of filled samples is lower compared to the pure matrix polymer (Figure 4-15). The $\tan \delta$ is the ratio of loss modulus to storage modulus. This shows that the elasticity of the polymer is increased stronger than the viscosity through the addition of filler. When the glass transition was passed the polymer is in the rubber state and the specimen became too soft at some point and the measurement was stopped. The composites filled with functionalized CB show an enhanced dimensional stability compared to the non-functionalized CB. This results from additional reinforcement of the polymer-filler interactions, since the bound polymer entangles with the matrix polymer.

The given results show, that the functionalization of $\mathrm{CB}$ with surface bound polymer enhances its reinforcing properties, since the polymer-filler interactions are increased, whereas the filler-filler interactions are decreased. Especially for the VP copolymer-functionalized CB composite, a significant difference compared to non-functionalized $\mathrm{CB}$ is observable. 


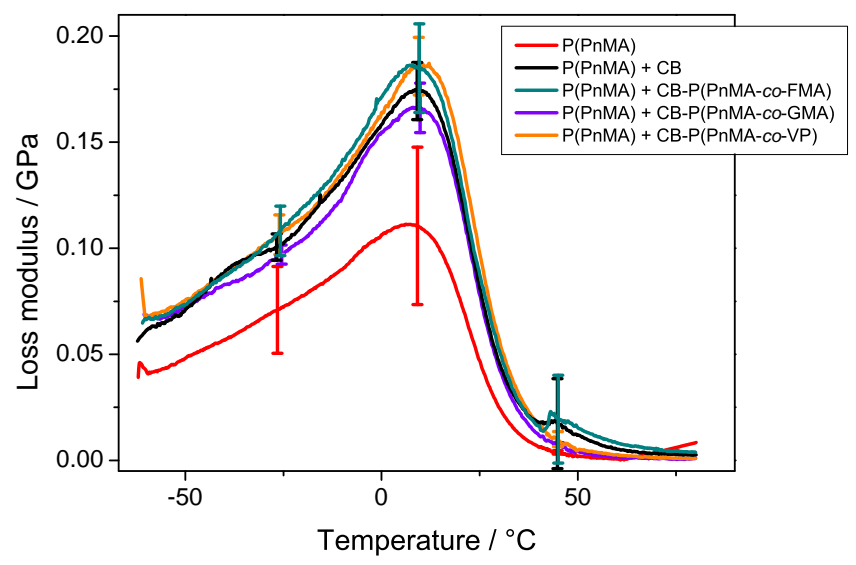

Figure 4-14: Temperature-dependent loss modulus of the pure matrix polymer PPnMA and of PPnMA composites filled with $10 \mathrm{wt} \%$ of untreated and functionalized CB.

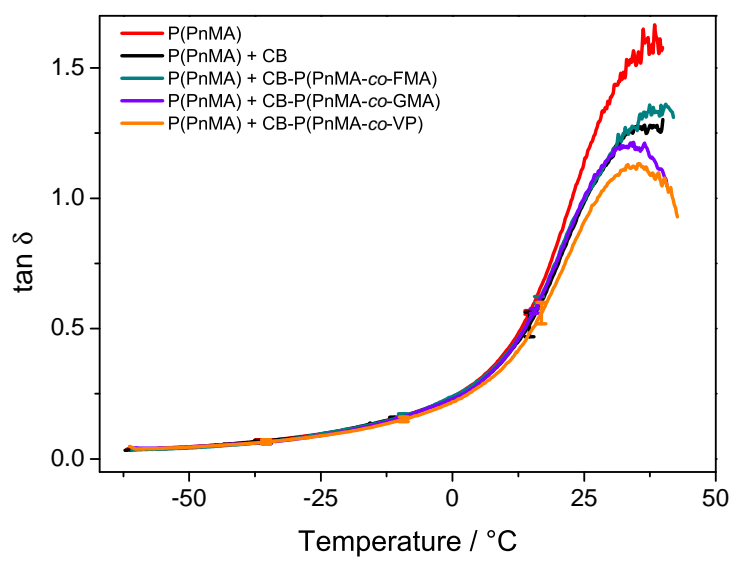

Figure 4-15: Temperature-dependent $\tan \delta$ of the pure matrix polymer PPnMA and of PPnMA composites filled with $10 \mathrm{wt} \%$ of untreated and functionalized $\mathrm{CB}$. 


\subsection{Functionalized CB as Filler in Tire Compounds}

To investigate the influence of the functionalization of $\mathrm{CB}$ on its properties as a reinforcing filler in tire compounds, the grafting-to approach needs to be conducted with butadiene copolymers. Therefore, suitable monomers with the anchor groups studied previously in this work will be presented and their copolymerization with butadiene studied. At least, the functionalization of CB with butadiene copolymers will be discussed.

\subsubsection{Synthesis of Butadiene Copolymers}

The miscibility of polymers depends on their interactions described by the Flory-Huggins theory. To ensure proper interactions between the surface bound copolymer and the matrix of the compound, the polymers need to mix and therefore be alike. ${ }^{[130]}$ As a consequence, the comonomer ratio within the copolymer should be little. Also, the surface bound copolymer should have a $T_{\mathrm{g}}$ similar to the matrix polymer. Therefore, the synthesis of terpolymers consisting of butadiene, styrene and the anchor group containing comonomers might be favorable.

For the synthesis of butadiene copolymers containing furan and epoxide anchor groups styrene based-comonomers were synthesized. The structures of glycidyl 4-vinylbenzyl ether (GMSt) and 4-furfuryloxymethylstyrene (FMSt) are shown in Figure 4-16. Besides the direct introduction of the anchor groups during copolymerization, a post-functionalization of the copolymer

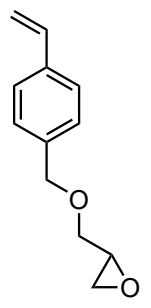

GMSt

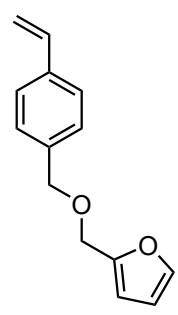

FMSt<smiles>C=Cc1ccc(CCl)cc1</smiles>

CMSt

Figure 4-16: Structures of styrene based monomers glycidyl 4vinylbenzyl ether (GMSt), 4-furfuryloxymethylstyrene (FMSt) and 4(chloromethyl)styrene (CMSt) for copolymerization with butadiene. 
Table 4-12: Number average molecular mass, dispersity and comonomer ratio of $\mathrm{P}(\mathrm{Bd}-\mathrm{co}-\mathrm{St}-\mathrm{co}-\mathrm{X})$ before and after the polymerization. SEC analysis was conducted using the Mark-Houwink coefficients of polybutadiene. The copolymer ratios were determined via ${ }^{1} \mathrm{H}-\mathrm{NMR}$ analysis. The terpolymerizations were performed with BPTT and ACCN in toluene at $87^{\circ} \mathrm{C}$ for $24 \mathrm{~h}$ and with a monomer-to-RAFT-to-initiator ratio of 3700:1:1.7. The copolymerization of Bd and VP was performed with CDSPA as RAFT agent and ACCN in toluene at $87^{\circ} \mathrm{C}$ for $24 \mathrm{~h}$ and with a monomer-to-RAFT-to-initiator ratio of 3000:1:1.7.

\begin{tabular}{|c|c|c|c|c|c|c|}
\hline \multirow[b]{2}{*}{$X$} & & \multicolumn{3}{|c|}{$x / \mathrm{mol} \%$} & \multirow[b]{2}{*}{$\bar{M}_{\mathrm{n}} / 10^{4} \mathrm{~g} \mathrm{~mol}^{-1}$} & \multirow[b]{2}{*}{$Ð$} \\
\hline & & $\mathrm{Bd}$ & St & $\mathrm{X}$ & & \\
\hline \multirow{2}{*}{ FMSt } & monomer & 73 & 17 & 10 & & \\
\hline & polymer & - & - & - & - & - \\
\hline \multirow{2}{*}{ GMSt } & monomer & 73 & 24 & 3 & & \\
\hline & polymer & 68 & 25 & 7 & 1.4 & 1.6 \\
\hline \multirow{4}{*}{ CMSt } & monomer & 73 & 17 & 10 & & \\
\hline & polymer & 36 & 28 & 36 & 2.8 & 3.3 \\
\hline & monomer & 73 & 24 & 3 & & \\
\hline & polymer & 57 & 20 & 23 & 1.1 & 3.0 \\
\hline \multirow{6}{*}{ VP } & monomer & 73 & 17 & 10 & & \\
\hline & polymer & 50 & 26 & 24 & 1.0 & 3.3 \\
\hline & monomer & 73 & 24 & 3 & & \\
\hline & polymer & 58 & 25 & 17 & 0.7 & 2.7 \\
\hline & monomer & 90 & - & 10 & & \\
\hline & polymer & 87 & - & 13 & 1.3 & 1.7 \\
\hline
\end{tabular}

is possible too. Therefore, 4-(chloromethyl)styrene (CMSt, Figure 4-16) was also used as comonomer for later O-etherification. [107:131] The advantage of this approach is, that the copolymer can be functionalized with a variety of anchor groups. Terpolymerizations were conducted with a constant ratio of butadiene under variation of the styrene-to-comonomer ratio.

Table 4-12 gives an overview of the copolymer compositions before and after the polymerization and the results from SEC analysis. Since the copolymer containing FMSt could not be re-dissolved, analysis was not possible. 
This is most likely due to crosslinking during the polymerization through Diels-Alder reaction of the furan unit and butadiene double bonds.

The copolymerization of GMSt resulted in a copolymer with $7 \mathrm{~mol} \%$ of the comonomer and a dispersity of 1.7. The butadiene ratio is lower than in the monomer mixture, indicating that the comonomer radical favors the addition of styrene monomers and vice versa.

This tendency is even more distinct for the CMSt comonomer. Both polymerizations gave copolymers with a much higher CMSt and lower butadiene ratio than in the monomer mixture. Also, the dispersity of both copolymers was over 3 , which is very high for RAFT polymerizations. Since the copolymerization of FMSt and butadiene did not work, the copolymerization with CMSt should be investigated further. The favored copolymer composition could be achieved by increasing the butadiene ratio, but may be ending up with a gradient copolymer instead of a statistical one.

From the results of $\mathrm{CB}$ functionalization with methacrylate copolymers vinylpyridine was the most interesting comonomer. Like the copolymers containing CMSt, the ratio of VP was higher and butadiene ratio was lower than in the monomer mixture. The dispersities close to 3 indicating bad control of the polymerization by the RAFT agent BPTT. Additionally, the copolymerization seems to be slow, resulting in a low $\bar{M}_{\mathrm{n}}$.

Overall, the terpolymerizations yield polymers with high dispersities. Therefore a copolymerization of $\mathrm{Bd}$ and VP without additional styrene was conducted. The resulting $\mathrm{P}(\mathrm{Bd}-\mathrm{co}-\mathrm{VP})$ has a comonomer ratio of $\mathrm{VP}$ of $13 \mathrm{~mol} \%$, which is only little higher than in the monomer mixture and so it is most likely a statistical copolymer. Also, the use of CDSPA as RAFT agent let to better control of the polymerization, resulting in a dispersity of 1.7. Additionally, a higher molar mass compared to the VP-containing terpolymers was achieved.

\subsubsection{Functionalization of CB with Butadiene Copolymers}

The results of $\mathrm{CB}$ functionalization with anchor groups-containing copolymers showed that pyridine is a suitable anchor group. The functionalization was more or less independent of the grafting conditions and the resulting hybrid material might also have self-healing properties due to the non-covalent grafting mechanism. Moreover, the oxidation of CB particles let to an increase of the grafting density of the vinylpyridine copolymer. Therefore, $\mathrm{CB}$ and $\mathrm{CB}-\mathrm{COOH}$ particles functionalized with the copolymer $\mathrm{P}(\mathrm{Bd}-\mathrm{co}-\mathrm{VP})$ were synthezised for application as fillers in tire compounds. 


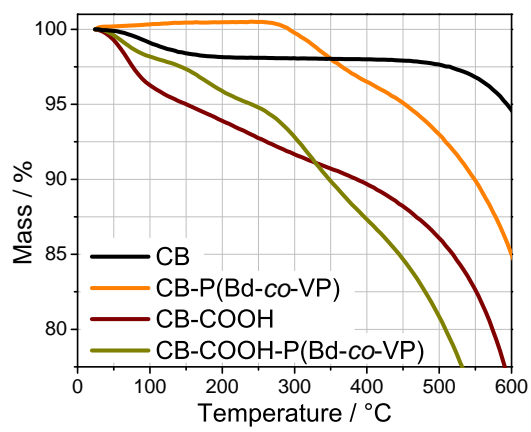

Figure 4-17: Thermograms of untreated $\mathrm{CB}$, oxidized $\mathrm{CB}$ (CB-COOH) and copolymer-functionalized $\mathrm{CB}$ (CB-P(Bd-co-VP) and (CB-COOH$\mathrm{P}(\mathrm{Bd}-\mathrm{co}-\mathrm{VP})$ ) measured under $\mathrm{N}_{2}$ atmosphere.

Table 4-13: Weight percentage of carbon, hydrogen and nitrogen measured by EA and weight loss between 250 and $450{ }^{\circ} \mathrm{C}$ measured by TGA under $\mathrm{N}_{2}$ atmosphere of untreated $\mathrm{CB}$, oxidized $\mathrm{CB}(\mathrm{CB}-\mathrm{COOH})$ and copolymer-functionalized CB (CB-P(Bd-co-VP) and (CB-COOH-P(Bdco-VP)). The copolymer P(Bd-co-VP) $\left(\bar{M}_{\mathrm{n}}=1.3 \cdot 10^{4} \mathrm{~g} \mathrm{~mol}^{-1}, Ð=1.7\right.$, $x_{\mathrm{VP}}=13 \mathrm{~mol} \%$ ) used for functionalization was synthesized via RAFT polymerization (Subsection 6.4.1). The functionalization was conducted at room temperature for $16 \mathrm{~h}$ in cyclohexane with a CB-to-copolymer mass ratio of $3: 2$.

\begin{tabular}{lcccc}
\hline & $\mathrm{C}$ & $\mathrm{H}$ & $\mathrm{N}$ & Weight loss \\
\cline { 2 - 5 } & \multicolumn{5}{c}{$/$ wt $\%$} \\
\hline CB & 96.56 & $<0.5$ & $<0.3$ & 0.9 \\
CB-P(Bd-co-VP) & 95.35 & 1.53 & 0.65 & 5.4 \\
CB-COOH & 83.49 & 0.79 & 0.61 & 4.5 \\
CB-COOH-P(Bd-co-VP) & 85.05 & 2.63 & 1.29 & 10.1 \\
\hline
\end{tabular}

The functionalization was conducted in cyclohexane at room temperature for $16 \mathrm{~h}$. Cyclohexane was chosen as solvent since it is a good solvent for the CB particles and the copolymer. The successful functionalization was confirmed via TGA and EA. The thermograms of the purified and dried particles before and after the functionalization are depicted in Figure 4-17. Both curves exhibit a distinct weight loss between 250 and $450{ }^{\circ} \mathrm{C}$ after 
the functionalization, which can be assigned to the decomposition of the copolymer (Figure G.1). The results of EA in Table 4-13 show an increase of the hydrogen and nitrogen weight percentage after the functionalization. These increases can be attributed to the copolymer.

The comparison of the weight loss before and after the functionalization (Table 4-13) shows that the amount of grafted copolymer, which is the difference of the weight loss of $\mathrm{CB}$ particles before and after the functionalization, is only marginally higher for $\mathrm{CB}-\mathrm{COOH}$ compared to $\mathrm{CB}$. This is in contrast to the results obtained with methacrylate copolymers, whose amount of grafted vinylpyridine copolymer was always approximately 1.5 times higher by the use of oxidized CB (Section 4.3 and Subsection 4.4.3). One explanation for this may be the fact that the interactions between the copolymer and the $\mathrm{CB}$ surface have changed by the use of butadiene as comonomer. While the interactions between the CB surface and the polar methacrylate polymers might be enhanced by the oxidation of $\mathrm{CB}$, it could be reduced in case of the non-polar butadiene. Nevertheless, under inclusion of the molar mass of the copolymer the grafting density of the VP copolymer grafted to untreated CB is equal to that obtained with the corresponding methacrylate copolymer.

\subsection{Conclusion and Future Perspectives}

The functionalization of CB via a grafting-to approach with anchor groupscontaining copolymers was studied in detail. Furan, epoxide and pyridine units were chosen as anchor groups, since their proposed grafting mechanisms differ from each other but are all promising. The furan unit is bound via Diels-Alder reaction to the CB surface. Grafting of the epoxide anchor group occurs via ring opening reaction with surface bound hydroxy groups. In contrast to these covalent binding mechanisms, the pyridine anchor is grafted via non-covalent $\pi-\pi$ interactions and hydrogen bonds. In addition, $\mathrm{CB}$ particles were oxidized to enhance the amount of oxygen functional groups at the surface.

The syntheses of respective methacrylate copolymers with FMA, GMA and VP as anchor group-containing monomers were conducted successfully. The investigation of the grafting conditions revealed some insights into the binding mechanisms and demonstrated the suitability of the anchor groups for the functionalization of $\mathrm{CB}$. It was found for the functionalization of untreated $\mathrm{CB}$ that the grafting density of the copolymer is almost independent of the anchor group and the grafting conditions, i.e. reaction time and 
temperature. Along with the finding that even a small portion of polymer without an appropriate anchor group is grafted to $\mathrm{CB}$, those results allow the conclusion that at least parts of the copolymers are grafted to the CB surface only via adsorption.

The functionalization of untreated and oxidized CB with the furan anchor was only successful when using untreated CB. This confirms the proposed binding mechanism via Diels-Alder reaction. The aromatic system of CB is disturbed through oxidation and the Diels-Alder reaction can not proceed. Furthermore, this result is interesting with regard to the above mentioned adsorption of polymer to the CB surface. Since no weight loss of FMA copolymers after the reaction was observed in the thermograms of both oxidized CB particles, also adsorption of polymer did not occur. This can be assigned to alteration of the interactions between polymer and CB surface through oxidation. Still, the results of the conducted experiments do not allow to conclude if the copolymer is covalently bound completely or only partially to the CB surface via the furan anchor group. Within the investigation of the solution behavior of the CB particles those functionalized with the FMA copolymer started to settle down shortly after the non-functionalized CB particles. In contrast, the CB particles functionalized with GMA and VP copolymers remained stably dispersed for much longer. This might point out that only parts of the copolymer are covalently bound to the CB surface in case of the furan anchor group.

The situation is similar for the functionalization of CB with the epoxide anchor. The comparison of the weight loss after functionalization of untreated and oxidized CB particles only showed a significant difference for reactions at $120^{\circ} \mathrm{C}$. At lower reaction temperatures the weight loss was equal for all samples. As for the solution behavior, the oxidized CB particles functionalized with GMA copolymer remained dispersed over a longer period than the untreated ones, although the amount of grafted copolymer was lower. Overall, those results support the proposed binding mechanism of the epoxide group to the CB surface. On the one hand, the ring opening reaction might need some additional energy in the form of temperature to proceed. On the other hand, the fraction of covalently bound copolymer is increased by the oxidation of CB particles and simultaneously the fraction of copolymer grafted via adsorption to the surface is reduced. Further investigations may include the use of graphene instead of CB particles. Graphene is a crystalline allotrope of carbon without further functional groups, which can be oxidized like CB. Without the presence of any functional groups at the surface, a copolymer with epoxide anchor groups can not react with 
graphene. The difference of the amount of grafted polymer to untreated and oxidized graphene can thus be assigned to polymer bound covalently to the surface. This could be used for finding the optimum grafting conditions for the epoxide anchor, i.e. reaction time and temperature.

The results of the functionalization of CB with the VP copolymer showed that the reaction is fast and proceeds without the need of elevated temperatures. Through oxidation of the CB surface the grafting density is increased, which is most probably based on the formation of additional hydrogen bonds. Although the grafting is only non-covalent, the interactions between the pyridine anchor and the CB surface seem to be very strong. This can be observed in its solution behavior and in the results of DMA measurements. In contrast to all the other samples, both untreated and oxidized CB particles functionalized with VP copolymer remained dispersed in THF for over a week. The DMA measurements of PPnMA compounds filled with CB particles showed that both storage and loss modulus are increased through the functionalization of the CB with VP copolymer. Meaning, the reinforcing properties of $\mathrm{CB}$ are improved. Since the grafting of the pyridine anchor is fast, it could also be performed in situ during mixing of a rubber compound. Another advantage of this anchor group over the furan and epoxide units lies in the grafting mechanism. In contrast to covalent bonds, the non-covalent interactions may be easily renewed after breakage and so it could be interesting regarding self-healing properties of hybrid materials.

To investigate the influence of the functionalization of $\mathrm{CB}$ on its properties as reinforcing filler, further mechanical tests need to be conducted. This includes for example DMA measurements and tensile testing. Thereby copolymer properties like the comonomer ratio and the chain length should be varied. Besides statistical copolymers, also the use of block copolymers for the functionalization might be interesting to enable the precise positioning of the anchoring region within the polymer chain. Usually, the RAFT polymerization is a powerful tool for the synthesis of block copolymers but in case of methacrylate block copolymers it has one deficiency: because of the kinetics of the RAFT mechanism, in most monomer combinations the methacrylate monomers need to be polymerized as the first block, since most transfer constants of macroRAFT agents in methacrylate polymerizations are low. ${ }^{[132]}$ While this still enables the synthesis of AB block copolymers, it hinders the synthesis of ABA block copolymers if an anchor group-containing monomer like vinylpyridine should be placed inside the polymer chain (Figure H.1). Thus, in case of the block copolymerization with vinylpyridine, at least either another class of the comonomer or another polymerization 
technique need to be used.

The main goal of this work was the investigation of the polymer-filler interactions within tire compounds filled with functionalized nanoparticles. Therefore, the functionalization of CB via the grafting-to approach had to be conducted with butadiene copolymers. As comonomers styrene based-monomers containing the furan (FMSt) and epxoide (GMSt) anchor groups were synthesized. Additionally, CMSt was also used as comonomer because it can be modified with anchor groups after the polymerization via O-etherification.

At first, the synthesis of terpolymers of the anchor groups-containing monomers with butadiene and styrene was studied. Thereby, a fraction of styrene was supposed to be replaced by the anchor groups-containing monomers. The copolymerization of FMSt did succeed but the polymer could not be redissolved and analyzed. This can be assigned to crosslinking during the polymerization via Diels-Alder reaction of the furan units and the butadiene double bonds. For the copolymerizations of VP and CMSt it was found that the terpolymers have high dispersities and the monomer ratio within the polymer is shifted in contrast to the monomer mixture in expense of butadiene, i.e. the copolymerization of styrene with VP and CMSt is favored. Only in the case of GMSt as comonomer a low dispersity and a low ratio of the comonomer within the polymer was achieved. Since the copolymerization of FMSt and butadiene resulted in a crosslinked copolymer, further investigation should focus on the copolymerisation of butadiene with CMSt. Due to the post-modification of the CMSt copolymer, it gives access to a wide range of anchor groups.

The functionalization of $\mathrm{CB}$ with a butadiene-based copolymer was conducted with $\mathrm{P}(\mathrm{Bd}-\mathrm{co}-\mathrm{VP})$. The synthesis of the respective copolymer resulted in a dispersity of 1.7 and a monomer ratio equal to the initial ratio in the monomer mixture. The functionalization of both untreated and oxidized CB was conducted at room temperature. For the untreated CB a grafting density of the copolymer equal to that of VP methacrylate copolymers was obtained. In contrast, the increase of the grafting density by the use of oxidized CB particles, which had been observed before, was only marginally. This may be due to deterioration of the interactions between the copolymer, which consists mostly of non-polar butadiene units, and the CB surface, whose polarity is increased through oxidation. Nevertheless, the functionalization of untreated and oxidized CB with a VP butadiene copolymer was successful, resulting in potential fillers for tire compounds. 
For the demonstration of the suitability of the copolymer-functionalized $\mathrm{CB}$ particles as reinforcing filler and for the investigation of the resulting filler-filler and polymer-filler interactions, model compounds were supposed to be mixed and physically tested by Continental. Thereby, in addition to SBR compounds, also natural rubber (NR) compounds filled with the VP copolymer-functionalized CB particles were supposed to be mixed. NR is mainly used for truck tires and due to its chemical structure the interactions between CB and NR are strong. ${ }^{[14]}$ At the point this work was completed, the mixing of the compounds was already done but the results of the physical tests were not available and therefore can not be discussed.

Finally, the determination of the Mark-Houwink coefficients of PPnMA and the investigation of the radical polymerization kinetics of PnMA were studied in this work for the first time. ${ }^{\left[{ }^{[0]} \text { The }\right.}$ MHC were determined via a new approach using RAFT polymerization. The determination of the MHC always requires the knowledge of the molecular mass of the respective polymer samples. Here, an advantage of the RAFT mechanism was exploited, which allows the estimation of the number average molar mass of the polymer from the conversion and the initial concentrations of the RAFT agent and of the monomer. The resulting MHC were in the same order of magnitude as those of other $n$-alkyl methacrylates.

The propagation rate coefficients of PnMA in bulk and toluene solution were determined as a function of temperature by PLP-SEC experiments. The so-obtained data, in combination with the termination rate coefficients determined by L. Riemann [89], allow for the representation and reliable simulation of bulk PnMA polymerizations up to moderate degrees of monomer conversion. Additionally, the results support the family behavior of methacrylates in which bulk $k_{\mathrm{p}}$ increases with increasing size of the polymer side chain. In solution of toluene slightly lower $k_{\mathrm{p}}$ has been observed with the difference, however, occurring within the limits of experimental accuracy, i.e., within $15 \%$. This result offers new insights into the propagation kinetics of methacrylates in toluene solution, since no $k_{\mathrm{p}}$ data for other alkyl methacrylates in toluene solution have been reported with the exception of MMA. In contrast to PnMA, MMA was found to show an increase of $k_{\mathrm{p}}$ in toluene solution compared to bulk values. ${ }^{[125]}$ The results for PnMA and for MMA suggest that the same type of family behavior which had been found for acrylates in toluene solution with regard to the size of the polymer side chain will probably occur within the methacrylate family, too. ${ }^{[128]}$ This assumption remains to be investigated by PLP-experiments of other alkyl methacrylates in solution. 


\section{Closing Remarks}

Within this work it was shown that the RAFT polymerization is a powerful technique for the functionalization of two of the mostly used reinforcing fillers for rubber compounds, silica (Chapter 3) and CB (Chapter 4) particles, and thereby this enables both the improvement of the mechanical properties of filled compounds and the contribution to a better understanding of the interactions between fillers and the surrounding polymeric matrix.

Different strategies for the functionalization of the nanoparticles via grafting-to and grafting-from approaches were used, whereby it was taken advantage of the versatility of the RAFT technique. On the one hand, the RAFT mechanism is tolerant towards many functional groups and enables the polymerization of anchor groups-containing monomers. On the other hand, the RAFT agent itself can act as anchor group in order to directly graft the polymer to a surface.

The latter was used for the functionalization of silica nanoparticles in both grafting-from and grafting-to approaches. The results of physical tests of tire compounds filled with these silica nanoparticles demonstrated that the functionalization is a promising method for the improvement of the mechanical properties of such compounds. In this respect it should be emphasized that based on the results these fillers may offer new possibilities for the adjustment of the mechanical properties than classical systems like the silica-silane technology do. Overall, the results of the physical tests pointed out that the polymer-filler interactions within the compounds were increased most probably through the entanglement of the surface bound polymer with the polymeric matrix. This is reflected in the improvement of the rebound 70 and in the abrasion. Even though the compounds had much higher crosslink distances compared to compounds filled with silanized silica nanoparticles, the abrasion was comparable or even lower. This indicates 
that the network strength is increased significantly through the functionalization of the silica nanoparticles. However, no significant reduction of the filler-filler interactions was observed. This may be assigned to insufficient shielding of the silica nanoparticles by the surface bound polymer. Either the grafting density of the polymer was too low or the polymer chains are mostly stretched into the matrix leaving only a little fraction of polymer wrapped around the silica surface. This remains to be investigated by the variation of both chain length and grafting density of the surface bound polymer.

The CB particles were functionalized in a grafting-to approach with copolymers containing anchor groups within the polymer chain. Furan, epoxide and pyridine anchor groups were chosen due to their different grafting mechanisms. The investigation of the grafting conditions revealed new insights into these mechanisms, which can be used for the precise functionalization of $\mathrm{CB}$ with the copolymers to tune its properties as reinforcing filler in rubber compounds. Thereby it was found that the furan anchor group can solely bind to untreated $\mathrm{CB}$, which has only a small amount of functional group at the surface. The oxidation of CB prevents the Diels-Alder reaction because the aromaticity of the CB surface is disturbed. The results of the epoxide anchor group showed that the grafting via ring opening reaction is at least favored by external energy input, i.e. by elevated temperatures. In contrast, the non-covalent interactions between the pyridine anchor group and the CB surface seem to be very strong, because the grafting occurred fast and independent of temperature.

The grafting density of the methacrylate copolymers at untreated CB particles was approximately equal independent of the anchor group and the grafting conditions, time and temperature. This might be assigned to a maximum loading capacity of the CB surface. Since this should be proportional to the specific surface area of the $\mathrm{CB}$ particles, this assumption could be tested by the variation of the size of $\mathrm{CB}$ particles. It was further found that a part of the polymer is only grafted to the CB via adsorption at the surface. This is important for the discussion of the results and for the tuning of the functionalization especially by the use of anchor groups with covalent grafting mechanisms.

There are two questions arising from these results: What portion of the polymer is purely adsorbed at the surface and trough how many anchor groups is one polymer chain bound covalently to the surface? Both questions are important for the use of functionalized CB particles as filler in rubber compounds, concerning the influence of the grafted polymer on the fillerfiller and polymer-filler interactions within the compound. Purely adsorbed 
polymer could reduce the filler-filler interactions, but might not enhance the polymer-filler interactions significantly. If the polymer chains are bound via several anchor groups to the surface it might also not be able to interact strongly with the polymeric matrix trough entanglement, resulting in the same outcome. To ensure that the surface bound polymer is able to interact with the surrounding polymeric matrix the fraction of the anchor groupscontaining monomer should be low, so multiple binding of one polymer chain to the CB surface might be prevented. Additionally, instead of the use of random copolymers, the use of block copolymer with short blocks of the anchor groups-containing monomers might be interesting too.

The situation might be different for the pyridine anchor group. On the one hand, the non-covalent interactions between the anchor group and the CB surface seem to be very strong. On the other hand, this bond can still be broken much more easily compared to covalent bonds. Therefore, it remains interesting to learn more about the influence of such functionalized CB particles on the filler-filler and polymer-filler interactions within filled compounds. Also, the self-healing capability of the pyridine anchor group might improve the mechanical properties and the durability of the compounds. A first step towards answering all of these questions was done within this work by the preparation of CB particles functionalized with $\mathrm{P}(\mathrm{Bd}-\mathrm{co}-\mathrm{VP})$ to be used as filler in tire compounds. The results of the physical tests of the compounds were still pending at the time this work was finished. 



\section{Experimental Section}

\subsection{Chemicals}

2,2'-Azobis(2-methylpropionitrile) (AIBN, Sigma Aldrich, 98\%) and 1,1'azobis(cyclohexanecarbonitrile) (ACCN, Sigma Aldrich, 98\%) were recrystallized from methanol. Pentyl methacrylate (PnMA, ABCR), furfuryl methacrylate (FMA, Sigma Aldrich, 97\%), hexyl methacrylate (HMA, Sigma Aldrich, $98 \%$ ), glycidyl methacrylate (GMA, Sigma Aldrich, 97\%), 4-vinylpyridine (VP, Sigma Aldrich, $95 \%$ ), 4-(chloromethyl)styrene (CMSt, Sigma Aldrich, $90 \%$ ) and styrene (St, Sigma Aldrich, 99\%) were passed through a basic alumina column prior to use. All commercially available solvents and chemicals were used in highest purity and without further purification.

\subsection{Synthesis of Monomers}

\subsubsection{Glycidyl 4-vinylbenzyl ether (GMSt)}<smiles>C=Cc1ccc(COCC2CO2)cc1</smiles>

GMSt was synthesized following the procedure published by Weston et al.. [131]

${ }^{1} \mathrm{H}-\mathrm{NMR}\left(300 \mathrm{MHz}, \mathrm{CDCl}_{3}\right) \delta(\mathrm{ppm})=2.62(\mathrm{dd}, J=2.7,5.0 \mathrm{~Hz}, 1 \mathrm{H}, \mathrm{Gly})$, 2.80 (dd, $J=4.1,5.0 \mathrm{~Hz}, 1 \mathrm{H}$, Gly), 3.19 (ddt, $J=3.0,4.2,5.8 \mathrm{~Hz}, 1 \mathrm{H}$, Gly), 
3.43 (dd, $J=5.8,11.4 \mathrm{~Hz}, 1 \mathrm{H}, \mathrm{O}-\mathrm{CH}_{2}$-Gly), 3.76 (dd, $J=3.0,11.4 \mathrm{~Hz}, 1 \mathrm{H}$, O-CH 2 -Gly), 4.49-4.66 (m, 2H, O-CH $-\mathrm{Ph}$ ), 5.24 (dd, $J=1.0,10.9 \mathrm{~Hz}, 1 \mathrm{H}$, $\mathrm{CH}_{2}$-CH-Ph), 5.74 (dd, $J=1.0,17.6 \mathrm{~Hz}, 1 \mathrm{H}, \mathrm{CH}_{2}$-CH-Ph), 6.72 (dd, $J=10.9$, $17.6 \mathrm{~Hz}, 1 \mathrm{H}, \mathrm{CH}_{2}-\mathrm{CH}-\mathrm{Ph}$ ), 7.24-7.45 (m, 4H, $\mathrm{CH}_{\mathrm{Ar}}$ ).

\subsubsection{4-Furfuryloxymethylstyrene (FMSt)}

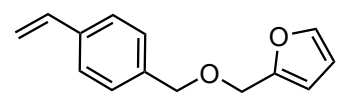

FMSt was synthesized following the procedure published by Goussé et al.. [107]

${ }^{1} \mathrm{H}-\mathrm{NMR}\left(300 \mathrm{MHz}, \mathrm{CDCl}_{3}\right) \delta$ (ppm) $=4.49$ (s, 2H, O-CH - -Fur), 4.54 (s, $2 \mathrm{H}, \mathrm{O}-\mathrm{CH}_{2}-\mathrm{Ph}$ ), 5.25 (dd, $J=1.0,10.9 \mathrm{~Hz}, 1 \mathrm{H}, \mathrm{CH}_{2}-\mathrm{CH}-\mathrm{Ph}$ ), 5.75 (dd, $J=1.0,17.6 \mathrm{~Hz}, 1 \mathrm{H}, \mathrm{CH}_{2}-\mathrm{CH}-\mathrm{Ph}$ ), 6.31-6.39 (m, 2H, Fur), 6.72 (dd, $\left.J=10.9,17.6 \mathrm{~Hz}, 1 \mathrm{H}, \mathrm{CH}_{2}-\mathrm{CH}-\mathrm{Ph}\right), 7.32-7.45$ (m, $5 \mathrm{H}, \mathrm{CH}_{\mathrm{Ar}}$, Fur).

\subsection{Synthesis of RAFT Agents}

\subsubsection{Benzylpropyltrithiocarbonate (BPTT)}<smiles>CCCSC(=S)SCc1ccccc1</smiles>

BPTT was synthesized following the procedure published by Rotzoll et al. . ${ }^{[40]}$

${ }^{1}$ H-NMR $\left(300 \mathrm{MHz}, \mathrm{CDCl}_{3}\right) \delta(\mathrm{ppm})=0.93\left(\mathrm{t}, J=7.4 \mathrm{~Hz}, 3 \mathrm{H}, \mathrm{CH}_{3}\right), 1.6$ (h, $J=7.4 \mathrm{~Hz}, 2 \mathrm{H}, \mathrm{CH}_{2}$ ), 3.26 (t, $J=7.4 \mathrm{~Hz}, 2 \mathrm{H}, \mathrm{S}-\mathrm{CH}_{2}$ ), 4.52 (s, 2H, Ph$\left.\mathrm{CH}_{2}\right), 7.10-7.29\left(\mathrm{~m}, 5 \mathrm{H}, \mathrm{CH}_{\mathrm{Ar}}\right)$. 


\subsubsection{Activated 4-Cyano-4-[(dodecylsulfanylthiocarbonyl)} sulfanyl]pentanoic acid (Act. CDSPA)<smiles>CC(C)(C)CSC(=S)SC(C)(C#N)CCC(=O)N1CCSC1=S</smiles>

The activation of CDSPA was performed according to the method in literature using CDSPA instead of CPDB. ${ }^{[34]}$ Silica gel column chromatography was carried out with a 3:2 mixture of hexane and ethyl acetate.

${ }^{1} \mathrm{H}-\mathrm{NMR}\left(300 \mathrm{MHz}, \mathrm{CDCl}_{3}\right) \delta(\mathrm{ppm})=0.80\left(\mathrm{~m}, 3 \mathrm{H}, \mathrm{CH}_{3}\right), 1.19(\mathrm{~m}, 18 \mathrm{H}$, S- $\mathrm{CH}_{2}-\mathrm{CH}_{2}-\mathrm{C}_{9} \mathrm{H}_{18}-\mathrm{CH}_{3}$ ), 1.60 (m, 2H, S- $\mathrm{CH}_{2}-\mathrm{CH}_{2}$ ), 1.82 (d, 3H, $\mathrm{CH}_{3}$ ), 1.9 (m, $\left.2 \mathrm{H}, \mathrm{C}(\mathrm{O}) \mathrm{CH}_{2} \mathrm{CH}_{2}\right), 2.48$ (m, $\left.2 \mathrm{H}, \mathrm{C}(\mathrm{O}) \mathrm{CH}_{2} \mathrm{CH}_{2}\right), 3.25$ (m, 4H, N-CH , $\mathrm{S}-\mathrm{CH}_{2}-\mathrm{C}_{11} \mathrm{H}_{23}$ ), 3.51 (m, 2H, S-CH $-\mathrm{CH}_{2}-\mathrm{N}$ ).

6.3.3 Dodecyl-((trimethoxysilyl)ethylphenylmethyl) trithiocarbonate (DTPT)<smiles>COCCc1ccc(CSC(=S)SCC(C)(C)C)cc1</smiles>

DTPT was synthesized analogue to PTPT as described by Rotzoll using 1dodecanethiol instead of 1-propanethiol. ${ }^{[40]}$

${ }^{1} \mathrm{H}-\mathrm{NMR}\left(300 \mathrm{MHz}, \mathrm{CDCl}_{3}\right) \delta(\mathrm{ppm})=0.79\left(\mathrm{~s}, 3 \mathrm{H}, \mathrm{CH}_{3}\right), 0.78-0.98(\mathrm{~m}$, $2 \mathrm{H}, \mathrm{Si}-\mathrm{CH}_{2}$ ), 1.18 (s, $20 \mathrm{H}, \mathrm{S}-\mathrm{CH}_{2}-\mathrm{C}_{1} \mathrm{OH}_{20}-\mathrm{CH}_{3}$ ), 1.60 (pd, $J=8.2,7.2 \mathrm{~Hz}, 2 \mathrm{H}$, Si- $\left.\mathrm{CH}_{2}-\mathrm{CH}_{2}\right), 3.20-3.39\left(\mathrm{~m}, 2 \mathrm{H}, \mathrm{S}-\mathrm{CH}_{2}-\mathrm{C}_{1} 1 \mathrm{H}_{23}\right), 3.46(\mathrm{td}, J=1.6,0.4 \mathrm{~Hz}$, 9H, O-CH 3 ), 4.48 (d, $2 \mathrm{H}, J=3.2 \mathrm{~Hz}, \mathrm{Ph}-\mathrm{CH}_{2}$ ), 6.95-7.22 (m, 4H, $\mathrm{CH}_{\mathrm{Ar}}$ ). 
6.3.4 Propyl-((trimethoxysilyl)ethylphenylmethyl) trithiocarbonate (PTPT)<smiles>CCCSC(=O)SCc1ccc(COC)cc1</smiles>

PTPT was synthesized as described by Rotzoll. [40]

${ }^{1}$ H-NMR $\left(300 \mathrm{MHz}, \mathrm{CDCl}_{3}\right) \delta(\mathrm{ppm})=0.83-1.00\left(\mathrm{~m}, 5 \mathrm{H}, \mathrm{CH}_{3}, \mathrm{Si}^{-\mathrm{CH}_{2}}\right)$, 1.54-1.76 (m, $2 \mathrm{H}, \mathrm{CH}_{2}$ ), 2.53-2.69 (m, $2 \mathrm{H}, \mathrm{Si}_{-}-\mathrm{CH}_{2}-\mathrm{CH}_{2}$ ), 3.21-3.33 (m, $2 \mathrm{H}$, $\mathrm{S}-\mathrm{CH}_{2}-\mathrm{C}_{5} \mathrm{H}_{6}$ ), $3.48\left(\mathrm{t}, J=1.8 \mathrm{~Hz}, 9 \mathrm{H}, \mathrm{O}-\mathrm{CH}_{3}\right), 4.44-4.54\left(\mathrm{~m}, 2 \mathrm{H}, \mathrm{Ph}-\mathrm{CH}_{2}\right.$ ), 6.97-7.25 (m, $\left.4 \mathrm{H}, \mathrm{CH}_{\mathrm{Ar}}\right)$.

\subsection{RAFT Polymerizations}

\subsubsection{Butadiene Copolymers}

The synthesis of butadiene copolymers was performed in an high-pressure autoclave as described by Springer. ${ }^{[11]}$ Polymerizations were conducted at $87^{\circ} \mathrm{C}$ for $24 \mathrm{~h}$ using toluene as solvent and ACCN as initiator.

SBR polymerizations were performed using the $1900 \mathrm{~mL}$ reaction vessel setup with an amount of $240 \mathrm{~g}$ of butadiene (Table 6-1).

Terpolymers were synthezised in the $450 \mathrm{~mL}$ vessel $(20 \mathrm{~g}$ butadiene, $30 \mathrm{~mL}$ toluene) using BPTT as RAFT agent. The monomer-RAFT-ACCN ratio of 3700:1:1.7 was fixed under variation of the monomer fractions. 
Table 6-1: Composition of butadiene copolymerizations in the $1900 \mathrm{~mL}$ vessel.

\begin{tabular}{lllll}
\hline & $\mathrm{M}_{2}$ & RAFT & Bd: $\mathrm{M}_{2}:$ R:I & $V($ tol $) / \mathrm{mL}$ \\
\hline SBR & St & BPTT & $2700: 1000: 1: 1.7$ & 350 \\
SBR-CDSPA & St & act. CDSPA & $2700: 1000: 1: 1.7$ & 350 \\
P(Bd-co-VP) & VP & CDSPA & $2700: 300: 1: 1.7$ & 500 \\
GF-RAFT & St & Si-RAFT (21.5 g) & $2700: 1000:-: 1.7$ & 350 \\
\hline
\end{tabular}

\subsubsection{Methacrylate Homo- and Copolymers}

In a typical procedure monomer(s), RAFT agent, AIBN and solvent were mixed, purged with argon for $10 \mathrm{~min}$ and heated to $60^{\circ} \mathrm{C}$. The polymerization was stopped by cooling and exposure to air. Conversion was determined gravimetrically after removing residual monomer(s) and solvent in vacuo at $60{ }^{\circ} \mathrm{C}$ for $24 \mathrm{~h}$. The polymerization conditions of each sample are stated in the respective part within this work.

\subsection{Synthesis and Functionalization of Silica Nanoparticles}

Silica nanoparticles provided by PlasmaChem ( $50 \mathrm{wt} \%$ aq. solution, particle diameter: $20 \mathrm{~nm}$ ) were precipitated in hexane, centrifuged, washed three times with methanol and dried in vacuo at ambient temperature for $24 \mathrm{~h}$.

\subsubsection{Synthesis of Silica Nanoparticles via Stöber Method}

A mixture of ethanol $(395 \mathrm{~mL}, 6.70 \mathrm{~mol})$, methanol $(530 \mathrm{~mL}, 13.1 \mathrm{~mol})$, ammonia (32 wt\%, $29.5 \mathrm{~mL}, 0.50 \mathrm{~mol})$, water $(26.6 \mathrm{~mL}, 1.50 \mathrm{~mol})$ and tetraethyl orthosilicate $(33.2 \mathrm{~mL}, 0.20 \mathrm{~mol})$ was stirred vigorously at ambient temperature for $24 \mathrm{~h}$. The solution was concentrated in vacuo. The remaining solution was centrifuged $\left(9000 \mathrm{rpm}, 0^{\circ} \mathrm{C}, 30 \mathrm{~min}\right)$, the particles were washed two times with methanol and were dried in vacuo at ambient temperature for $12 \mathrm{~h}$. 


\subsubsection{Immobilization of RAFT Agent with Anchor Group}

Silica nanoarticles (PlasmaChem, $18.0 \mathrm{~g}$ ) were dispersed in 1,2-dimethoxyethane $(300 \mathrm{~mL})$ under sonication for $30 \mathrm{~min}$. The RAFT agent (PTPT or DPTP, $2.6 \mathrm{mmol})$ and saturated aq. solution of maleic anhydride $(0.3 \mathrm{~mL})$ were added. The solution was stirred vigorously at ambient temperature for $3 \mathrm{~d}$. The solution was centrifuged $\left(9000 \mathrm{rpm}, 0^{\circ} \mathrm{C}, 1 \mathrm{~h}\right.$ ), the particles (SiPTPT/Si-DPTP) were washed three times with methanol and dried in vacuo at ambient temperature for $3 \mathrm{~d}$.

\subsubsection{Immobilization of RAFT Agent without Anchor Group}

Silica nanoarticles (PlasmaChem, $18.0 \mathrm{~g}$ ) were dispersed in THF (dry, $300 \mathrm{~mL}$ ) under sonication for $30 \mathrm{~min}$. 3-Aminopropyldimethylethoxysilane (APDEMS, $2.0 \mathrm{~g}, 14.4 \mathrm{mmol}$ ) was added, the solution was purged with argon for $30 \mathrm{~min}$ and stirred vigorously at $90^{\circ} \mathrm{C}$ for $3 \mathrm{~d}$. The solution was centrifuged ( $9000 \mathrm{rpm}, 0^{\circ} \mathrm{C}, 1 \mathrm{~h}$ ), the particles (Si-APDMES) were washed two times with THF and dried in vacuo at ambient temperature for $3 \mathrm{~d}$.

Si-APDMES (18.0 g) and act. CDSPA (1.5 g) were dissolved in THF (dry, $200 \mathrm{~mL}$ ) under sonication for $30 \mathrm{~min}$. The solution was purged with argon for $30 \mathrm{~min}$ and stirred vigorously at ambient temperature for $3 \mathrm{~d}$. The solution was centrifuged $\left(9000 \mathrm{rpm}, 0^{\circ} \mathrm{C}, 1 \mathrm{~h}\right)$, the particles (Si-CDSPA) were washed three times with THF and dried in vacuo at ambient temperature for $3 \mathrm{~d}$.

\subsubsection{Grafting of Polymer to Silica Nanoparticles via Thiol-Ene Click Reaction}

Silica nanoarticles (Stöber Methode, $17.0 \mathrm{~g}$ ) were dispersed in toluene $(500 \mathrm{~mL})$ under sonication for $30 \mathrm{~min}$. Dimethylethoxyvinylsilane (DMEVS, $3.0 \mathrm{~mL}, 18.2 \mathrm{mmol}$ ) was added, the solution was purged with argon for $30 \mathrm{~min}$ and stirred vigorously at $80^{\circ} \mathrm{C}$ for $3 \mathrm{~d}$. The solution was centrifuged ( $9000 \mathrm{rpm}, 0^{\circ} \mathrm{C}, 1 \mathrm{~h}$ ), the particles (Si-DMEVS) were washed two times with toluene and dried in vacuo at $40^{\circ} \mathrm{C}$ for $12 \mathrm{~h}$.

SBR was dissolved in toluene $(300 \mathrm{~mL})$, hydrazine $(2.4 \mathrm{~mL})$ was added and the solution was stirred vigorously at ambient temperature for $2 \mathrm{~d}$. After evaporation of the solvent at ambient temperature the polymer (SBR-SH) was dried in vacuo at $40^{\circ} \mathrm{C}$ for $24 \mathrm{~h}$.

Si-DMEVS (19.0 g) and SBR-SH (40 g) were dissolved in toluene (500 mL) 
under sonication for $30 \mathrm{~min}$. ACCN (0.7 g, $2.8 \mathrm{mmol})$ was added, the solution was purged with argon for $30 \mathrm{~min}$, and stirred vigorously at $80^{\circ} \mathrm{C}$ for $2 \mathrm{~d}$. The solution was centrifuged $\left(9000 \mathrm{rpm}, 0^{\circ} \mathrm{C}, 1 \mathrm{~h}\right)$ and the particles (GT-Click) were washed several times with toluene.

\subsubsection{Grafting of Polymer to Silica Nanoparticles via Nucleophilic Substitution}

Silica nanoarticles (Stöber method, $17.0 \mathrm{~g}$ ) were dispersed in toluene $(500 \mathrm{~mL})$ under sonication for $30 \mathrm{~min}$. APDMES $(2.0 \mathrm{~g}, 14.4 \mathrm{mmol})$ was added, the solution was purged with argon for $30 \mathrm{~min}$ and stirred vigorously at $90^{\circ} \mathrm{C}$ for $36 \mathrm{~h}$. The solution was centrifuged $\left(9000 \mathrm{rpm}, 0^{\circ} \mathrm{C}, 1 \mathrm{~h}\right)$, the particles (Si-APDMES) were washed two times with toluene and dried in vacuo at $40^{\circ} \mathrm{C}$ for $12 \mathrm{~h}$.

Si-APDMES (19.0 g) and SBR-CDSPA (40 g) were dissolved in toluene (500 mL) under sonication for $30 \mathrm{~min}$. The solution was purged with argon for $30 \mathrm{~min}$ and stirred vigorously at $90^{\circ} \mathrm{C}$ for $3 \mathrm{~d}$. The solution was centrifuged (9000 rpm, $0^{\circ} \mathrm{C}, 1 \mathrm{~h}$ ) and the particles (GT-Subs) were washed several times with toluene.

\subsubsection{Thioether End Group}

The functionalized silica particles were dissolved in toluene $(300 \mathrm{~mL})$, hydrazine $(2.4 \mathrm{~mL})$ was added and the solution was stirred vigorously at ambient temperature for $2 \mathrm{~d}$. Butyl acrylate $(1.0 \mathrm{~mL}, 7.0 \mathrm{mmol})$ was added, after $3 \mathrm{~d}$ the solution was centrifuged $\left(9000 \mathrm{rpm}, 0^{\circ} \mathrm{C}, 1 \mathrm{~h}\right)$, the particles were washed two times with toluene and dried in vacuo at $40^{\circ} \mathrm{C}$ for $12 \mathrm{~h}$.

\subsection{Functionalization of Carbon Black}

Carbon Black (CORAX ${ }^{\circledR}, \mathrm{N} 220$, Orion Engineered Carbons, specific surface area: $106 \mathrm{~m}^{2} \mathrm{~g}^{-1}$ ) was purified by soxhlet extraction using toluene for $72 \mathrm{~h}$.

\subsubsection{Synthesis of $\mathrm{CB}-\mathrm{COOH}$}

CB $\left(100 \mathrm{~g} \mathrm{~L}^{-1}\right)$ was stirred in $\mathrm{HNO}_{3}\left(65 \%\right.$ aq.) at $100^{\circ} \mathrm{C}$ for $24 \mathrm{~h}$. The solution was centrifuged $\left(9000 \mathrm{rpm}, 15^{\circ} \mathrm{C}, 1 \mathrm{~h}\right)$, the particles $(\mathrm{CB}-\mathrm{COOH})$ were washed three times with water and dried in vacuo at $60^{\circ} \mathrm{C}$ for $24 \mathrm{~h}$. 


\subsubsection{Synthesis of $\mathrm{CB}-\mathrm{OH}$}

$\mathrm{CB}-\mathrm{OH}$ was synthezised analogue to a procedure published by Beckert et al. for graphite. [105]

\subsubsection{Grafting of Polymer to CB}

In a typical procedure polymer was dissolved in a good solvent (Table 6-2), CB was added and the dispersion was stirred at a respective temperature. After a certain period of time the dispersion was centrifuged $(9000 \mathrm{rpm}$, $15^{\circ} \mathrm{C}, 1 \mathrm{~h}$ ), the particles were washed three times with the used solvent and dried in vacuo at $60^{\circ} \mathrm{C}$ for $24 \mathrm{~h}$.

Table 6-2: Solvents used for grafting the respective copolymer to CB.

\begin{tabular}{ll}
\hline Polymer & Solvent \\
\hline Methacrylate copolymers & Dibenzyl ether \\
Butadiene copolymers & Cyclohexane \\
\hline
\end{tabular}

\subsection{Instrumentation}

\subsubsection{Density Measurement}

Density measurements were performed on an Anton Paar (DMA 60 and DMA 602TP) oscillating U-tube densitometer with a thermostated cell (Anton Paar, DMA 602H) using water as the heat-transfer fluid. The densitometer was calibrated with distilled water and with air. The measurements were performed at temperatures between 25.5 and $60.2^{\circ} \mathrm{C}$ in bulk and in toluene solution.

\subsubsection{Dynamic Mechanical Analysis}

The dynamic mechanical analysis (DMA) was performed in single cantilever geometry on a DMA 8000 by Perkin Elmer with a heating rate of $2 \mathrm{~K} \mathrm{~min}^{-1}$ under constant strain. Samples were either measured in material pockets 
(stainless steel, Perkin Elmer) or as test specimen produced by injection moulding (HAAKE MiniJet Pro, Thermo Scientific).

\subsubsection{Elemental Analysis}

Elemental analysis (EA) was performed by the analytical lab of the institute of inorganic chemistry of the Georg-August-University Göttingen on a 4.1 Vario EL 3 by Elementar. The samples were measured twice and the mean value is given.

\subsubsection{Nuclear Magnetic Resonance Spectroscopy}

${ }^{1} \mathrm{H}$-Nuclear magnetic resonance $\left({ }^{1} \mathrm{H}-\mathrm{NMR}\right)$ spectra were measured with a Varian Unity 300 instrument at room temperature. The samples were dissolved in deuterated chloroform. Residual solvent proton signals were used as internal standard.

\subsubsection{PLP-SEC}

Pulsed laser polimerizations (PLP) were carried out using an ATLEX-I Laser (ATL Lasertechnik $\mathrm{GmbH}$, operated on the XeF line at $351 \mathrm{~nm}$; maximum pulse energy: $7 \mathrm{~mJ}$, pulse width: $20 \mathrm{~nm}$, maximum p.r.r.: $1000 \mathrm{~Hz}$,). Laser irradiation was performed by using two cylindrical plano-concave lenses (Thorelabs, UV-grade-fused-silica, antireflective coating for $290-370 \mathrm{~nm}$, reflectance $<0.5 \%$ per surface; lense 1 : LK4326-UV, $f_{\mathrm{L}}=-25.0 \mathrm{~mm}$; lense 2: LK4385-UV, $f_{\mathrm{L}}=-75.0 \mathrm{~mm}$ ) and two cylindrical plano-convex lenses (lense 3: Thorelabs, UV-grade-fused-silica, anti-reflective coating for 290-370 nm, reflectance $<0.5 \%$ per surface; LJ4395-UV, $f_{\mathrm{L}}=100 \mathrm{~mm}$; lense 4: Melles Griot, UV-grade-fused silica, V-type anti-reflective coating for 351,nm, reflectance $<0.5 \%$ per surface; SCX-25.4-101.7-UV-248-355, $f_{\mathrm{L}}=25.4 \mathrm{~mm}$ ). This equipment allows for widening the laser beam from 3 to $4 \times 6 \mathrm{~mm}$ to an area of $16 \times 16 \mathrm{~mm}$. The p.r.r.'s were chosen to be in the range of 3 to $10 \mathrm{~Hz}$. The polymerizing system was contained in a double-walled cylindrical cuvette (Starna, 65.14/Q/10, spectrosil-fused quartz, transmission $\geq 80 \%$ between 192 and $2700 \mathrm{~nm}$, path length: $10 \mathrm{~mm}$ ) which was thermostated (Haake, K, Haake F3) using ethylene glycol/water (4:1) as the heat-transfer fluid. 
PnMA, 2,2-dimethoxy-2-phenylacetophenone (DMPA) $\left(5.0 \mathrm{~mol} \mathrm{~L}^{-1}\right)$ and toluene were mixed, transferred into the cuvette, purged with argon for $10 \mathrm{~min}$ and pre-heated for $15 \mathrm{~min}$. Polymerization was stopped by pouring the reaction mixture into a flask containing HQ as the inhibitor. Monomer conversion was determined gravimetrically after removing residual monomer in vacuo at $60^{\circ} \mathrm{C}$.

\subsubsection{Size-Exclusion Chromatography}

The SEC measurements were performed on an Agilent Technology SEC setup consisting of an autosampler (Agilent 1260 ALS G1329B), an injector (Agilent 1260 ALS), a HPLC pump (PSS Agilent Technologies 1260 Iso Pump G1310B), a pre-column (PSS SDV, $8 \times 50 \mathrm{~mm}, 5 \mu \mathrm{m}$ particle size), three separation columns (PSS SDV, $8 \times 300 \mathrm{~mm}, 5 \mu \mathrm{m}$ particle size with pore sizes of $10^{6}, 10^{5}$ and $10^{3} \AA$ ), a UV detector (PSS Agilent Technologies 1260 VWDVL), and a refractive index (RI) detector (PSS Technologies $1260 \mathrm{RID}$ ). THF was used as the eluent with a flow rate of $1.0 \mathrm{~mL} \mathrm{~min}^{-1}$ at $35^{\circ} \mathrm{C}$ and toluene as the internal standard. The system was calibrated with poly(methyl methacrylate) standards of narrow molecular weight distribution purchased from PSS, Mainz ( $K=12.98 \cdot 10^{-3} \mathrm{~mL} \mathrm{~g}^{-1}, a=0.688$ ). Butadiene copolymers were analyzed using the Mark-Houwink coefficients of polybutadiene $\left(K=5.78 \cdot 10^{-2} \mathrm{~mL} \mathrm{~g}^{-1}, a=0.670\right)$.

\subsubsection{Thermogravimetric Analysis}

Thermogravimetric analysis (TGA) was conducted with a TG 209 F3 Tarsus by Netzsch. Samples were heated in aluminium oxide pot from room temperature to $1000 \mathrm{~K}$ with a heating rate of $10 \mathrm{Kmin}^{-1}$. Silica samples were measured using ambient air as purging gas, CB samples were measured under nitrogen atmosphere with a flow rate of $20 \mathrm{~mL} \mathrm{~min}^{-1}$.

\subsubsection{Transmission Electron Microscopy}

Transmission electron microscopy (TEM) was done at a Philips CM12 with an acceleration voltage of $120 \mathrm{kV}$ and a resolution of 2.4 angstrom. TEM images were recorded with a Olympus CCD camera with a resolution of 1376 x 1032 pxl.

For sample preparation a solution of $4 \mathrm{gL}^{-1}$ was sonicated for $30 \mathrm{~min}$, 
dropped onto a TEM grid (Plano, carbon film on copper mesh, $3.5 \mathrm{~mm}$, 400 mesh) and solvent was evaporated at ambient temperature. 



\section{Appendices}

\section{Appendix A}

TE Micrographs of GT-Si and GF-Si

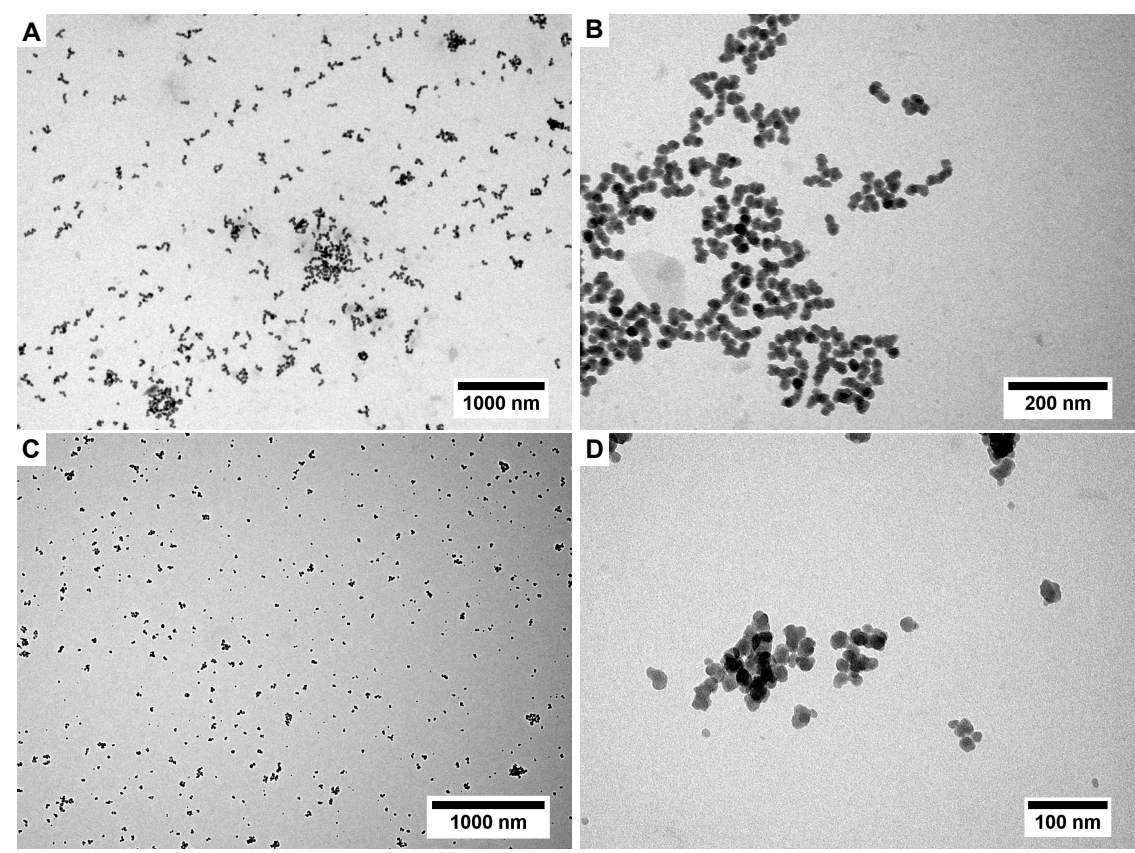

Figure A.1: TE micrographs of silica nanoparticles prepared via Stöber method (A and B) and purchased from PlasmaChem (C and D). 


\section{Appendices}

\section{Appendix B}

Thermogram of SBR

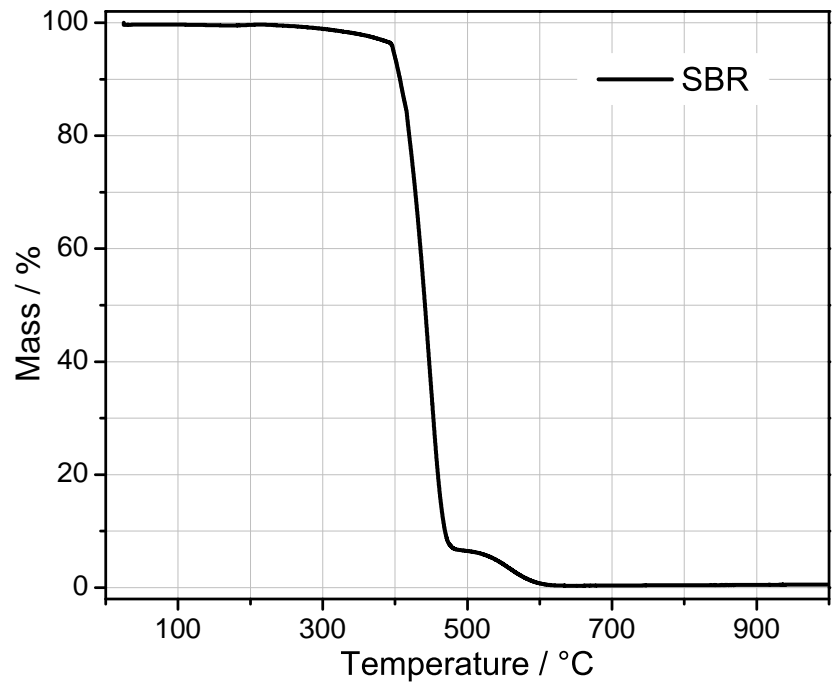

Figure B.1: Thermogram of SBR synthesized via RAFT polymerization measured under air. 


\section{Appendix C}

\section{Amount of Silica Samples Taken for Compound Mixing}

Table C.1: Actual amount of samples taken for mixing of silica compounds.

\begin{tabular}{lcccccc}
\hline & \multicolumn{5}{c}{ Amount of added particles / phr } \\
\cline { 2 - 7 } & 35 & 50 & 60 & 70 & 75 & 80 \\
\hline GT-Subs & 37.5 & & 64.2 & 75.0 & & \\
GT-Click & & 52.0 & 62.4 & 72.8 & & \\
GF-PTPT & & & 69.5 & 81.0 & & 92.6 \\
GF-CDSPA & & & 63.7 & 74.3 & 79.6 & \\
GF-DTPT & & 63.9 & 76.7 & 89.4 & & \\
\hline
\end{tabular}


Appendix D

Overview of the Results of Physical Tests of Compounds Containing Functionalized Silica Nanoparticles

The results presented in this section are part of an invention disclosure, which has already been submitted. [67] 
Table D.1: Results of the physical tests of cured compounds filled with VN3.

\begin{tabular}{|c|c|c|c|c|c|c|c|}
\hline & \multicolumn{4}{|c|}{$\mathrm{VN} 3+\mathrm{S}$} & \multicolumn{3}{|c|}{ VN3+L } \\
\hline & 60 & 70 & $70(2)$ & 80 & $70 / 8$ & $70 / 16$ & $70 / 24$ \\
\hline Density & & & & & & & \\
\hline $\begin{array}{l}\text { Density RT / } \mathrm{g} \mathrm{cm}^{-3} \\
\text { XDL }\end{array}$ & 1.128 & 1.152 & 1.152 & 1.174 & 1.140 & 1.131 & 1.124 \\
\hline $\begin{array}{l}\text { Dist. of Crosslinks } 1 \text { / nm } \\
\text { Shore Hardness }\end{array}$ & 9.3 & 8.3 & 8.6 & 8.1 & 9.9 & 8.7 & 9.7 \\
\hline $\begin{array}{l}\text { Hardness RT / ShA } \\
\text { Rebound }\end{array}$ & 54.6 & 56.7 & 59.0 & 62.6 & 53.6 & 50.4 & 50.4 \\
\hline Rebound RT / \% & 45.8 & 42.4 & 42.3 & 37.7 & 41.5 & 46.2 & 45.7 \\
\hline Rebound $70^{\circ} \mathrm{C} / \%$ & 64.0 & 60.7 & 61.3 & 58.9 & 55.8 & 58.7 & 58.1 \\
\hline Stress-Strain RT & & & & & & & \\
\hline M100 RT / MPa & 2.2 & 2.6 & 3.4 & 3.3 & 1.8 & 1.7 & 2.1 \\
\hline M300 RT / MPa & 7.5 & 8.9 & 10.1 & 10.3 & 5.2 & 5.0 & 5.7 \\
\hline DMA $55^{\circ} \mathrm{C}$ & & & & & & & \\
\hline$E^{\prime}(0.15 \%) / \mathrm{MPa}$ & 5.0 & 6.0 & 6.4 & 7.9 & 5.8 & 5.5 & 5.7 \\
\hline$E^{\prime}(8 \%) / \mathrm{MPa}$ & 4.2 & 4.7 & 5.0 & 5.8 & 4.4 & 4.4 & 4.7 \\
\hline $\begin{array}{l}E^{\prime}(0.15 \%)-E^{\prime}(8 \%) / \mathrm{MPa} \\
\text { DMA const. Force }\end{array}$ & 0.8 & 1.2 & 1.4 & 2.1 & 1.5 & 1.1 & 1.0 \\
\hline$T \tan \delta \max$ & -18 & -18 & -18 & -18 & -20 & -23 & -23 \\
\hline $\tan \delta\left(-15^{\circ} \mathrm{C}\right)$ & 1.024 & 0.969 & 0.970 & 0.874 & 0.940 & 0.863 & 0.768 \\
\hline $\tan \delta\left(70{ }^{\circ} \mathrm{C}\right)$ & 0.086 & 0.096 & 0.106 & 0.125 & 0.147 & 0.141 & 0.121 \\
\hline Abrasion & & & & & & & \\
\hline Abrasion RT / $\mathrm{mm}^{3}$ & 173 & 144 & 129 & 144 & 207 & 262 & 188 \\
\hline
\end{tabular}



GT-Si.

\begin{tabular}{|c|c|c|c|c|c|c|c|c|}
\hline & \multicolumn{5}{|c|}{ GT-Si } & \multicolumn{3}{|c|}{ GT-Si+L } \\
\hline & 35 & 50 & 60 & 70 & 80 & $70 / 8$ & $70 / 16$ & $70 / 24$ \\
\hline Density & & & & & & & & \\
\hline $\begin{array}{l}\text { Density RT / } \mathrm{g} \mathrm{cm}^{-3} \\
\text { XDL }\end{array}$ & 1.046 & 1.083 & 1.106 & 1.130 & 1.148 & 1.121 & 1.118 & 1.111 \\
\hline $\begin{array}{l}\text { Dist. of Crosslinks } 1 \text { / nm } \\
\text { Shore Hardness }\end{array}$ & 10.5 & 12.4 & 11.2 & 10.9 & 13.7 & 11.5 & 10.3 & 10.1 \\
\hline $\begin{array}{l}\text { Hardness RT / ShA } \\
\text { Rebound }\end{array}$ & 39.4 & 42.0 & 44.4 & 44.7 & 48.3 & 46.8 & 47.0 & 46.3 \\
\hline Rebound RT / \% & 56.0 & 55.3 & 51.6 & 49.7 & 48.3 & 50.8 & 51.6 & 52.0 \\
\hline Rebound $70^{\circ} \mathrm{C} / \%$ & 73.1 & 71.3 & 67.9 & 63.9 & 60.0 & 65.4 & 65.2 & 65.0 \\
\hline Stress-Strain RT & & & & & & & & \\
\hline M100 RT / MPa & 0.8 & 1.0 & 1.1 & 1.3 & 1.3 & 1.3 & 1.4 & 1.4 \\
\hline $\begin{array}{l}\text { M300 RT / MPa } \\
\text { DMA } 55^{\circ} \mathrm{C}\end{array}$ & 1.8 & 2.2 & 2.3 & 2.7 & 2.9 & 3.0 & 3.2 & 3.3 \\
\hline$E^{\prime}(0.15 \%) / \mathrm{MPa}$ & 2.5 & 2.8 & 2.9 & 3.8 & 4.1 & 3.4 & 3.9 & 3.3 \\
\hline$E^{\prime}(8 \%) / \mathrm{MPa}$ & 2.4 & 2.6 & 2.6 & 3.4 & 3.5 & 3.1 & 3.5 & 3.0 \\
\hline $\begin{array}{l}E^{\prime}(0.15 \%)-E^{\prime}(8 \%) / \mathrm{MPa} \\
\text { DMA const. Force }\end{array}$ & 0.1 & 0.1 & 0.2 & 0.4 & 0.6 & 0.4 & 0.4 & 0.3 \\
\hline$T \tan \delta \max$ & -20 & -20 & -20 & -20 & -20 & -20 & -23 & -23 \\
\hline $\tan \delta\left(-15^{\circ} \mathrm{C}\right)$ & 1.176 & 1.106 & 1.091 & 1.025 & 1.012 & 1.012 & 0.980 & 0.929 \\
\hline $\tan \delta\left(70^{\circ} \mathrm{C}\right)$ & 0.049 & 0.067 & 0.079 & 0.123 & 0.129 & 0.114 & 0.096 & 0.090 \\
\hline Abrasion & & & & & & & & \\
\hline Abrasion RT / mm ${ }^{3}$ & 690 & 576 & 470 & 505 & 525 & 465 & 448 & 474 \\
\hline
\end{tabular}


Table D.3: Results of the physical tests of cured compounds filled with GT-Click and GT-Subs.

\begin{tabular}{|c|c|c|c|c|c|c|}
\hline & \multicolumn{3}{|c|}{ GT-Click } & \multicolumn{3}{|c|}{ GT-Subs } \\
\hline & 50 & 60 & 70 & 35 & 60 & 70 \\
\hline \multicolumn{7}{|l|}{ Density } \\
\hline Density RT / $\mathrm{g} \mathrm{cm}^{-3}$ & 1.088 & 1.109 & 1.133 & 1.054 & 1.111 & 1.132 \\
\hline \multicolumn{7}{|l|}{ XDL } \\
\hline \multicolumn{6}{|l|}{ Shore Hardness } & 20.3 \\
\hline Hardness RT / ShA & 41.8 & 43.5 & 45.9 & 38.8 & 44.7 & 46.8 \\
\hline \multicolumn{7}{|l|}{ Rebound } \\
\hline Rebound RT / \% & 54.4 & 51.6 & 49.2 & 55.9 & 52.8 & 51.6 \\
\hline Rebound $70^{\circ} \mathrm{C} / \%$ & 72.7 & 69.2 & 68.0 & 74.3 & 69.3 & 69.1 \\
\hline \multicolumn{7}{|l|}{ Stress-Strain RT } \\
\hline M100 RT / MPa & 1.3 & 1.0 & 1.2 & 1.1 & 1.5 & 1.4 \\
\hline M300 RT / MPa & 2.8 & 2.2 & 2.5 & 2.7 & 3.4 & 2.9 \\
\hline \multicolumn{7}{|l|}{ DMA $55^{\circ} \mathrm{C}$} \\
\hline$E^{\prime}(0.15 \%) / \mathrm{MPa}$ & 2.6 & 3.0 & 3.3 & 2.4 & 2.8 & 3.6 \\
\hline$E^{\prime}(8 \%) / \mathrm{MPa}$ & 2.4 & 2.8 & 2.9 & 2.3 & 2.7 & 3.2 \\
\hline$E^{\prime}(0.15 \%)-E^{\prime}(8 \%) / \mathrm{MPa}$ & 0.2 & 0.2 & 0.4 & 0.1 & 0.2 & 0.3 \\
\hline \multicolumn{7}{|l|}{ DMA const. Force } \\
\hline$T \tan \delta \max$ & -20 & -20 & -20 & -22 & -20 & -20 \\
\hline $\tan \delta\left(-15^{\circ} \mathrm{C}\right)$ & 1.208 & 1.191 & 1.163 & 1.104 & 1.167 & 1.143 \\
\hline $\tan \delta\left(70^{\circ} \mathrm{C}\right)$ & 0.058 & 0.066 & 0.075 & 0.054 & 0.066 & 0.078 \\
\hline \multicolumn{7}{|l|}{ Abrasion } \\
\hline Abrasion RT / mm ${ }^{3}$ & 540 & 506 & 498 & 540 & 531 & 498 \\
\hline
\end{tabular}


Table D.4: Results of the physical tests of cured compounds filled with GF-Si.

\begin{tabular}{|c|c|c|c|c|c|c|c|}
\hline & \multicolumn{4}{|c|}{ GF-Si } & \multicolumn{3}{|c|}{ GF-Si+S } \\
\hline & 50 & 60 & 70 & 80 & 50 & 60 & 70 \\
\hline \multicolumn{8}{|l|}{ Density } \\
\hline $\begin{array}{l}\text { Density RT / } \mathrm{g} \mathrm{cm}^{-3} \\
\text { XDL }\end{array}$ & 1.090 & 1.127 & 1.148 & 1.170 & 1.130 & 1.155 & 1.179 \\
\hline $\begin{array}{l}\text { Dist. of Crosslinks } 1 / \mathrm{nm} \\
\text { Shore Hardness }\end{array}$ & 11.2 & 9.5 & 10.0 & 10.2 & 8.7 & 7.5 & 7.7 \\
\hline $\begin{array}{l}\text { Hardness RT / ShA } \\
\text { Rebound }\end{array}$ & 38.1 & 41.5 & 44.6 & 45.7 & 44.9 & 45.7 & 48.8 \\
\hline Rebound RT / \% & 53.3 & 51.3 & 50.7 & 50.6 & 52.4 & 51.0 & 48.7 \\
\hline Rebound $70^{\circ} \mathrm{C} / \%$ & 71.1 & 65.2 & 61.9 & 59.2 & 68.6 & 65.2 & 65.7 \\
\hline Stress-Strain RT & & & & & & & \\
\hline M100 RT / MPa & 0.9 & 1.0 & 1.2 & 1.4 & 1.3 & 1.5 & 1.8 \\
\hline $\begin{array}{l}\text { M300 RT / MPa } \\
\text { DMA } 55^{\circ} \mathrm{C}\end{array}$ & 1.9 & 2.3 & 2.6 & 2.9 & 3.1 & 3.4 & 3.8 \\
\hline$E^{\prime}(0.15 \%) / \mathrm{MPa}$ & 2.3 & 2.7 & 3.0 & 3.9 & 3.5 & 3.7 & 4.0 \\
\hline$E^{\prime}(8 \%) / \mathrm{MPa}$ & 2.2 & 2.5 & 2.8 & 3.4 & 3.2 & 3.4 & 3.6 \\
\hline $\begin{array}{l}E^{\prime}(0.15 \%)-E^{\prime}(8 \%) / \mathrm{MPa} \\
\text { DMA const. Force }\end{array}$ & 0.1 & 0.2 & 0.2 & 0.5 & 0.3 & 0.3 & 0.4 \\
\hline$T \tan \delta \max$ & -20 & -23 & -20 & -23 & -20 & -20 & -20 \\
\hline $\tan \delta\left(-15^{\circ} \mathrm{C}\right)$ & 1.170 & 1.060 & 1.068 & 0.985 & 1.100 & 1.102 & 1.057 \\
\hline $\tan \delta\left(70^{\circ} \mathrm{C}\right)$ & 0.082 & 0.091 & 0.099 & 0.128 & 0.064 & 0.068 & 0.081 \\
\hline Abrasion & & & & & & & \\
\hline Abrasion RT / $\mathrm{mm}^{3}$ & 703 & 507 & 493 & 455 & 451 & 452 & 415 \\
\hline
\end{tabular}


Table D.5: Results of the physical tests of cured compounds filled with GF-CDSPA, GF-PTPT and GF-DTPT.

\begin{tabular}{|c|c|c|c|c|c|c|c|c|c|}
\hline & \multicolumn{3}{|c|}{ GF-CDSPA } & \multicolumn{3}{|c|}{ GF-PTPT } & \multicolumn{3}{|c|}{ GF-DTPT } \\
\hline & 60 & 70 & 75 & 60 & 70 & 80 & 50 & 60 & 70 \\
\hline Density & & & & & & & & & \\
\hline $\begin{array}{l}\text { Density RT } / \mathrm{g} \mathrm{cm}^{-3} \\
\text { XDL }\end{array}$ & 1.129 & 1.151 & 1.157 & 1.113 & 1.140 & 1.160 & 1.192 & 1.114 & 1.139 \\
\hline $\begin{array}{l}\text { Dist. of Crosslinks } 1 \text { / nm } \\
\text { Shore Hardness }\end{array}$ & 13.3 & 13.6 & 18.0 & 17.0 & 15.9 & 14.1 & 18.6 & 18.9 & 18.3 \\
\hline $\begin{array}{l}\text { Hardness RT / ShA } \\
\text { Rebound }\end{array}$ & 43.7 & 45.5 & 45.6 & 42.4 & 44.8 & 47.6 & 44.1 & 46.5 & 49.8 \\
\hline Rebound RT / \% & 52.1 & 50.8 & 50.5 & 52.7 & 50.2 & 50.0 & 50.6 & 50.6 & 49.0 \\
\hline $\begin{array}{l}\text { Rebound } 70{ }^{\circ} \mathrm{C} / \% \\
\text { Stress-Strain RT }\end{array}$ & 71.8 & 71.3 & 69.4 & 69.6 & 68.2 & 66.1 & 71.2 & 67.9 & 64.2 \\
\hline M100 RT / MPa & 1.2 & 1.4 & 1.4 & 1.2 & 1.4 & 1.7 & 1.4 & 1.6 & 2.0 \\
\hline $\begin{array}{l}\text { M300 RT / MPa } \\
\text { DMA } 55^{\circ} \mathrm{C}\end{array}$ & 3.0 & 3.2 & 3.2 & 3.1 & 3.6 & 4.0 & 3.0 & 3.2 & 3.6 \\
\hline$E^{\prime}(0.15 \%) / \mathrm{MPa}$ & 2.8 & 3.3 & 3.1 & 2.8 & 3.1 & 3.8 & 3.1 & 3.4 & 3.9 \\
\hline$E^{\prime}(8 \%) / \mathrm{MPa}$ & 2.6 & 3.0 & 2.8 & 2.6 & 2.8 & 3.5 & 2.9 & 3.1 & 3.5 \\
\hline $\begin{array}{l}E^{\prime}(0.15 \%)-E^{\prime}(8 \%) / \mathrm{MPa} \\
\text { DMA const. Force }\end{array}$ & 0.2 & 0.3 & 0.3 & 0.2 & 0.3 & 0.4 & 0.2 & 0.4 & 0.5 \\
\hline$T \tan \delta \max$ & -20 & -20 & -20 & -20 & -20 & -20 & -20 & -20 & -20 \\
\hline $\tan \delta\left(-15^{\circ} \mathrm{C}\right)$ & 1.118 & 1.130 & 1.107 & 1.150 & 1.153 & 1.063 & 1.089 & 1.089 & 1.085 \\
\hline $\begin{array}{l}\tan \delta\left(70{ }^{\circ} \mathrm{C}\right) \\
\text { Abrasion }\end{array}$ & 0.071 & 0.072 & 0.076 & 0.078 & 0.086 & 0.090 & 0.075 & 0.091 & 0.092 \\
\hline Abrasion RT / mm ${ }^{3}$ & 453 & 421 & 397 & 435 & 407 & 419 & 500 & 423 & 413 \\
\hline
\end{tabular}




\section{Appendix $\mathrm{E}$}

\section{MWDs of HMA Copolymers}
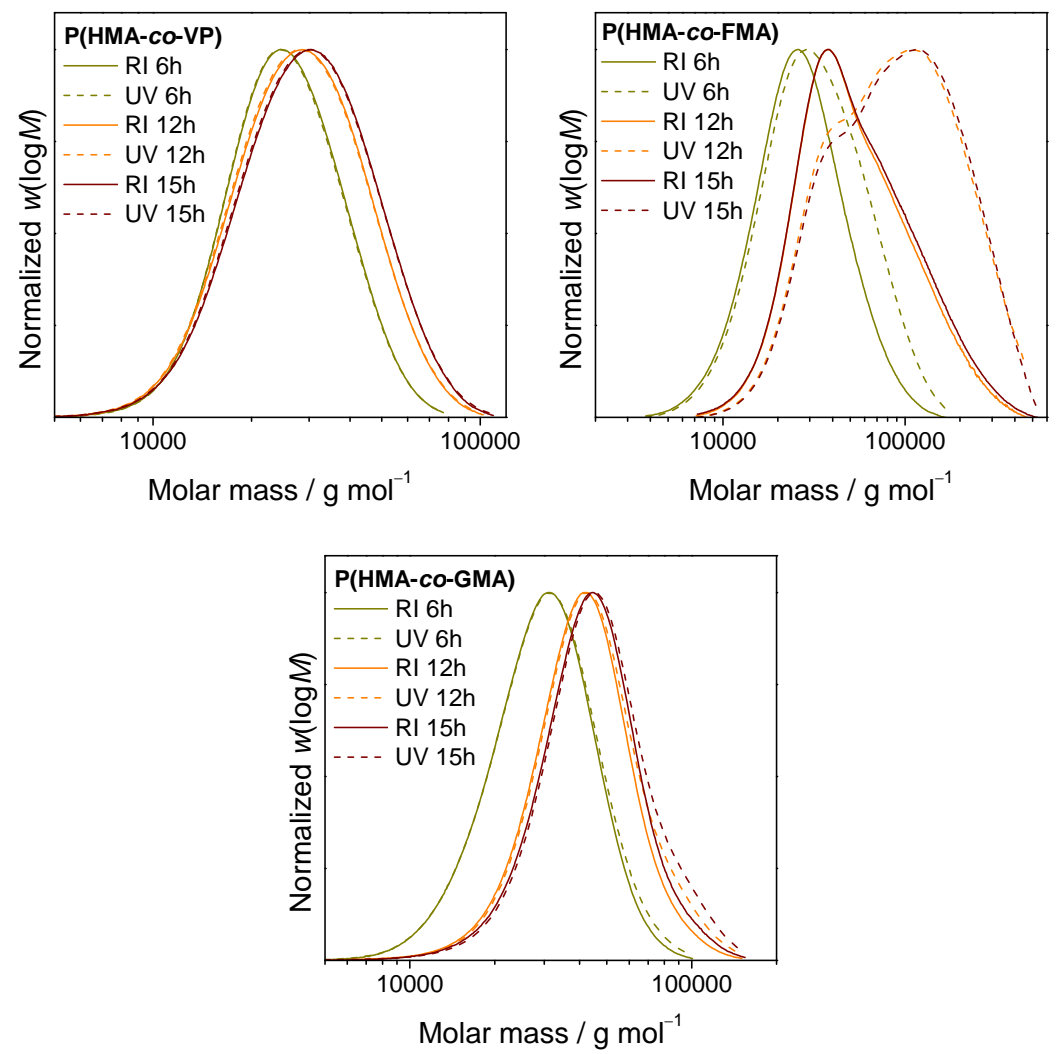

Figure E.1: Normalized molecular weight distributions of hexyl methacrylate copolymers after different polymerization periods obtained from SEC analysis using the Mark-Houwink coefficients of PHMA. Polymerizations were performed with CDSPA and AIBN in $50 \mathrm{wt} \%$ toluene at $60^{\circ} \mathrm{C}$ and with a monomer-to-RAFT-to-initiator ratio of 500:1:0.2 and $10 \mathrm{~mol} \%$ of the comonomer. 


\section{Appendix F}

\section{MWDs of PnMA Copolymers}

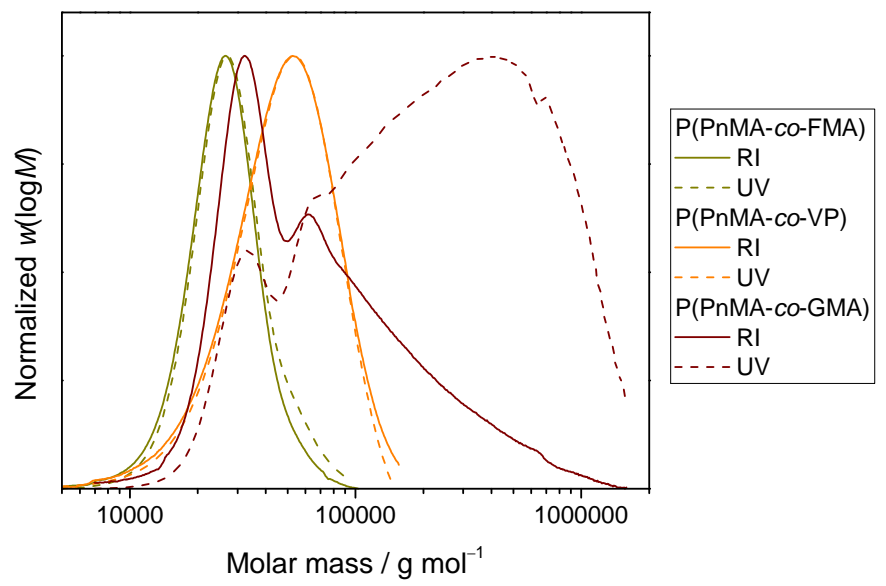

Figure F.1: Normalized molecular weight distributions of pentyl methacrylate copolymers obtained from SEC analysis using the MarkHouwink coefficients of PPnMA. Polymerizations were performed with CDSPA and AIBN in bulk at $60^{\circ} \mathrm{C}$, a monomer-to-RAFT-to-initiator ratio of 250:1:0.2 was used. The comonomer ratio was $10 \mathrm{~mol} \%$ in case of FMA and GMA and $5 \mathrm{~mol} \%$ in case of VP. 
Appendix G

Thermogram of $\mathrm{P}(\mathrm{Bd}-\mathrm{co}-\mathrm{VP})$

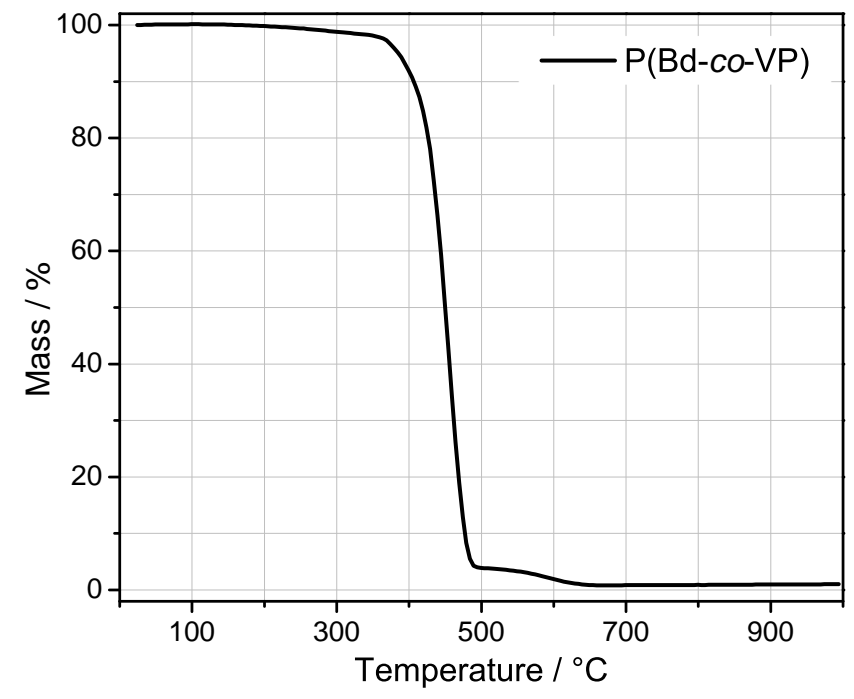

Figure G.1: Thermogram of $\mathrm{P}(\mathrm{Bd}-\mathrm{co}-\mathrm{VP})$ synthesized via RAFT polymerization measured under $\mathrm{N}_{2}$ atmosphere. 


\section{Appendix $\mathrm{H}$}

\section{Block Copolymerization of PnMA and VP}

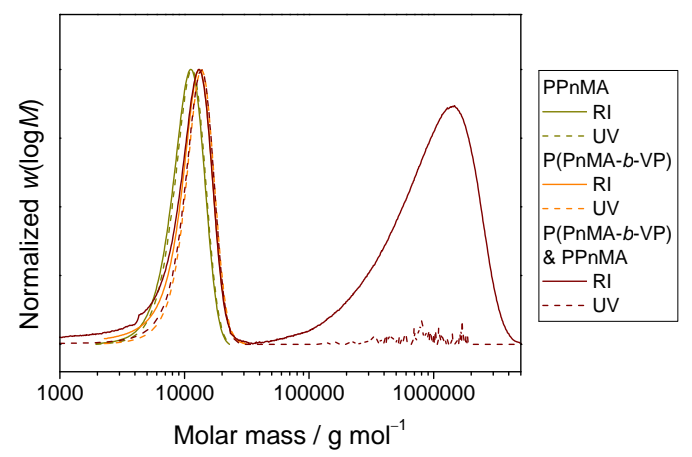

Figure H.1: Normalized molecular weight distributions of pentyl methacrylate homo- and copolymers obtained from SEC analysis using the Mark-Houwink coefficients of PPnMA. The polymerization of PPnMA was performed with CDSPA and AIBN in bulk at $60^{\circ} \mathrm{C}$ and a monomer-to-RAFT-to-initiator ratio of 80:1:0.1 was used. The resulting polymer was used as macroRAFT agent in a block copolymerization with VP with AIBN in $50 \mathrm{wt} \%$ toluene solution at $60^{\circ} \mathrm{C}$ and a monomerto-RAFT-to-initiator ratio of 50:1:0.1. The resulting block copolymer was used as macroRAFT agent in a subsequent block copolymerization with PnMA with AIBN in in $20 \mathrm{wt} \%$ toluene solution at $60^{\circ} \mathrm{C}$ and a monomer-to-RAFT-to-initiator ratio of 160:1:0.1.

The aim of this experiment was the synthesis of an ABA triblock copolymer of PnMA and VP, with VP being the block B in the middle of the polymer chain. The results from SEC analysis show, that the block copolymerization of P(PnMA- $b$-VP) via RAFT polymerization using PPnMA as macroRAFT agent was successful (also confirmed by NMR analysis). The subsequent block copolymerization of PnMA with P(PnMA- $b-\mathrm{VP})$ as macroRAFT agent did not succeed. The MWD of the macroRAFT agent did not change, but an additional peak at high molar masses occurred. Since this peak is only present in the RI signal, it can be assigned to PPnMA from a conventional radical polymerization. So it can be concluded, that the macroRAFT agent with the VP block is not suitable for PnMA block copolymerization. 



\section{Abbreviations}

\begin{tabular}{|c|c|}
\hline$a$ & Mark-Houwink coefficient \\
\hline$A$ & Arrhenius pre-exponential factor \\
\hline AIBN & 2,2'-azobis(2-methylpropionitrile) \\
\hline ACCN & $1,1^{\prime}$-azobis(cyclohexanecarbonitrile) \\
\hline APDMES & 3-aminopropyldimethylethoxysilane \\
\hline $\mathrm{Bd}$ & butadiene \\
\hline BMA & butyl methacrylate \\
\hline BPTT & benzylpropyltrithiocarbonate \\
\hline$c$ & concentration \\
\hline$c_{\mathrm{M}}^{0}$ & initial monomer concentration \\
\hline$c_{\mathrm{RAFT}}^{0}$ & initial RAFT concentration \\
\hline $\mathrm{CB}$ & carbon black \\
\hline $\mathrm{CB}-\mathrm{COOH}$ & oxidized CB mainly functionalized with carboxy groups \\
\hline $\begin{array}{l}\text { CB-COOH- } \\
\mathrm{P}(\mathrm{X})\end{array}$ & oxidized CB functionalized with a (co)polymer X \\
\hline $\mathrm{CB}-\mathrm{OH}$ & oxidized CB mainly functionalized with hydroxy groups \\
\hline $\mathrm{CB}-\mathrm{OH}-\mathrm{P}(\mathrm{X})$ & oxidized CB functionalized with a (co)polymer $\mathrm{P}(\mathrm{X})$ \\
\hline $\mathrm{CB}-\mathrm{P}(\mathrm{X})$ & untreated CB functionalized with a (co)polymer P(X) \\
\hline CDSPA & $\begin{array}{l}\text { 4-cyano-4-[(dodecylsulfanylthiocarbonyl)sulfanyl] pen- } \\
\text { tanoic acid }\end{array}$ \\
\hline CMSt & 4-(chloromethyl)styrene \\
\hline$\boxplus$ & dispersity \\
\hline$\delta$ & chemical shift, phase shift \\
\hline DBE & dibenzyl ether \\
\hline DMA & dodecyl methacrylate, dynamic mechanical analysis \\
\hline DMEVS & dimethylethoxyvinylsilane \\
\hline
\end{tabular}


DMPA 2,2-dimethoxy-2-phenylacetophenone

DTPT dodecyl-((trimethoxysilyl)ethylphenylmethyl) trithiocarbonate

$\varepsilon \quad$ strain

$E^{\prime} \quad$ storage modulus

$E^{\prime \prime} \quad$ loss modulus

$E_{\mathrm{A}} \quad$ activation energy

EA elemental analysis

EMA ethyl methacrylate

FMA furfuryl methacrylate

FMSt 4-furfuryloxymethylstyrene (FMSt)

GF grafting-from

GF-RAFT polymer-functionalized silica nanoparticles synthesized via RAFT polymerization in a grafting-from apporach using a particular RAFT agent (RAFT $=$ CDSPA, DTPT or PTPT)

GF-Si silica purchased by PlasmaChem used for functionalization of silica nanoparticles via grafting-from approach

GMA glycidyl methacrylate

GMSt glycidyl 4-vinylbenzyl ether

GT

GT-Si grafting-to

silica prepared via Stöber method used for functionalization of silica nanoparticles via grafting-to approach

GT-Click polymer-functionalized silica nanoparticles synthesized via thiol-ene click reaction in a grafting-to approach

GT-Subs polymer-functionalized silica nanoparticles synthesized via nucleophilic substitution in a grafting-to approach

HMA hexyl methacrylate

$\mathrm{HNO}_{3} \quad$ nitric acid

$1^{\bullet} \quad$ initiator radical

$k \quad$ rate coefficient

$k_{\mathrm{p}} \quad$ propagation rate coefficient

$K \quad$ Mark-Houwink coefficient

$L_{\mathrm{i}} \quad$ maxima of the first-derivative of the MWD

L, LBR liquid butadiene rubber 


$\begin{array}{ll}\text { M } & \text { monomer } \\ M & \text { molar mass } \\ M_{\text {theo. }} & \text { theoretical molar mass } \\ \bar{M}_{\mathrm{n}} & \text { number average molar mass } \\ \bar{M}_{\mathrm{v}} & \text { viscosity average molar mass } \\ \bar{M}_{\mathrm{w}} & \text { weight average molar mass } \\ \text { MHC } & \text { Mark-Houwink coefficients } \\ \text { MMA } & \text { methyl methacrylate } \\ \text { MWD } & \text { molar weight distribution } \\ \text { NMR } & \text { nuclear magnetic resonance spectroscopy } \\ \text { NR } & \text { natural rubber } \\ \text { P } & \text { polymer } \\ \mathrm{P}_{\mathrm{i}} & \text { polymer chain of length i } \\ \mathrm{P} & \text { polymer radical of length i } \\ \text { PHMA } & \text { poly(hexyl methacrylate) } \\ \text { phr } & \text { parts per hundred rubber } \\ \text { PLP-SEC } & \text { pulsed laser polymerization in conjunction with SEC } \\ \text { PMMA } & \text { poly(methyl methacrylate) } \\ \text { PnMA } & \text { pentyl methacrylate } \\ \text { PPnMA } & \text { poly(pentyl methacrylate) } \\ \text { p.r.r. } & \text { pulse repetetation rate } \\ \text { PTPT } & \text { propyl-(trimethoxysilyl)ethylphenylmethyl) trithiocar- } \\ & \text { bonate } \\ r & \text { copolymerization parameter } \\ \text { R } & \text { leaving group } \\ \text { R } & \text { radical of leaving group } \\ \rho & \text { density } \\ \text { RAFT } & \text { reversible addition-fragmentation chain transfer } \\ \text { RT } & \text { room temperature } \\ \text { S } & \text { silane } \\ \sigma & \text { stress } \\ \text { SBR } & \text { styrene butadiene rubber } \\ \text { SEC } & \text { size-exclusion chromatography } \\ \text { Si } & \text { silica } \\ \text { Si-APDMES } & \text { silica nanoparticles functionalized with APDMES } \\ \text { Si-DMEVS } & \text { silica nanoparticles functionalized with DMEVS } \\ & \end{array}$




$\begin{array}{ll}\text { Si-RAFT } & \text { silica nanoparticles functionalized with a RAFT agent } \\ \text { St } & \text { (RAFT }=\text { CDSPA, DTPT or PTPT) } \\ t & \text { time } \\ t_{0} & \text { inverse of p.r.r. } \\ T & \text { temperature } \\ T_{\mathrm{g}} & \text { glass transition temperature } \\ \tan \delta & \text { loss factor } \\ \text { TEM } & \text { transmission electron microscopy } \\ \text { TESPD } & \text { bis(triethoxysilylpropyl) disulfide } \\ \text { TGA } & \text { thermogravimetric analysis } \\ \text { THF } & \text { tetrahydrofuran } \\ U & \text { conversion } \\ \text { VN3 } & \text { standard silica } \\ \text { VP } & \text { 4-vinylpyridine } \\ x & \text { molar ratio } \\ x_{\mathrm{Co}} & \text { comonomer ratio } \\ \text { Z } & \text { stabilizing group }\end{array}$




\section{Bibliography}

[1] A. Rössler, G. Skillas, S. E. Pratsinis, Chemie in unserer Zeit 2001, 2001(1), 32-41.

[2] P. Ottersbach, C. Schmitz, J. Averdung, L. Heinrich, A. Gutsch, Chemie in Unserer Zeit 2005, 39(1), 54-59.

[3] F. S. F. Röthemeyer, Kautschuktechnologie: Werkstoffe - Verarbeitung Produkte, Carl Hanser Verlag, München, 2013.

[4] M. I. W. Baumann, Kautschuk und Gummi: Daten und Fakten zum Umweltschutz, Springer, Heidelberg, 2013.

[5] R. S. S. Thomas, Rubber Nanocomposites: Preparation, Properties and Applications, John Wiley \& Sons, Singapore, 2010.

[6] J. Liu, Y. Gao, D. Cao, L. Zhang, Z. Guo, Langmuir 2011, 27(12), 7926-7933.

[7] V. Mittal, Synthesis Techniques for Polymer Nanocomposites, Wiley, Heidelberg, 2015.

[8] Y. Wu, S. Wen, J. Shen, J. Jiang, S. Hu, L. Zhang, L. Liu, Radiation Physics and Chemistry 2015, 111, 91-97.

[9] A. V. J. C. A. Da Silva, N. Binde, P. Robert, S. Sterin, Rubber composition for tire comprising an organosilicon coupling system 2006, US9010393 B2.

[10] H. Kloppenburg, T. Groß, M. Mezger, C. Wrana, Chemie in Unserer Zeit 2009, 43(6), 392-406. 
[11] B. Springer, Polybutadien und Butadien enthaltende Copolymere mit kontrollierter Kettenstruktur durch RAFT Polymerisation, Dissertation, Georg-August-University Göttingen, 2011.

[12] D. Huebner, V. Koch, B. Ebeling, J. Mechau, J. E. Steinhoff, P. Vana, Journal of Polymer Science, Part A: Polymer Chemistry 2015, 53(1), 103-113.

[13] S. K. Kumar, N. Jouault, B. Benicewicz, T. Neely, Macromolecules 2013, 46(9), 3199-3214.

[14] C. Wortmann, F. Dettmer, F. Steiner, Chemie in Unserer Zeit 2013, 47(5), 300-309.

[15] L. Wu, U. Glebe, A. Böker, Macromolecules 2016, 49(24), 9586-9596.

[16] M. Rehahn, Chemie in unserer Zeit 2003, 37(1), 18-30.

[17] J. Roncali, Macromolecular Rapid Communications 2007, 28(17), $1761-1775$.

[18] M. R. Hill, R. N. Carmean, B. S. Sumerlin, Macromolecules 2015, 48(16), 5459-5469.

[19] G. Moad, E. Rizzardo, S. H. Thang, Australian Journal of Chemistry 2006, 59(10), 669.

[20] G. Moad, Polymer International 2017, 66(1), 26-41.

[21] J. Chiefari, Y. K. B. Chong, F. Ercole, J. Krstina, J. Jeffery, T. P. T. Le, R. T. A. Mayadunne, G. F. Meijs, C. L. Moad, G. Moad, E. Rizzardo, S. H. Thang, Macromolecules 1998, 31(16), 5559-5562.

[22] K. Matyjaszewski, Macromolecules 2012, 45(10), 4015-4039.

[23] J.-S. Wang, K. Matyjaszewski, Macromolecules 1995, 28(I), 79017910.

[24] G. Moad, E. Rizzardo, S. H. Thang, Polymer International 2011, 60(1), 9-25.

[25] B. Ebeling, P. Vana, Polymers 2011, 3(2), 719-739.

[26] H. Willcock, R. K. O'Reilly, Polymer Chemistry 2010, 1(2), 149-157. 
Bibliography

[27] S. Perrier, P. Takolpuckdee, C. A. Mars, Macromolecules 2005, 38(16), 6770-6774.

[28] G. Moad, E. Rizzardo, S. H. Thang, Australian Journal of Chemistry 2005, 58(6), 379-410.

[29] S. Perrier, P. Takolpuckdee, Journal of Polymer Science, Part A: Polymer Chemistry 2005, 43(22), 5347-5393.

[30] G. Gody, T. Maschmeyer, P. B. Zetterlund, Macromolecules 2014, 47, 639-649.

[31] J. Chiefari, R. Mayadunne, C. Moad, G. Moad, E. Rizzardo, A. Postma, M. Skidmore, S. H. Thang, Macromolecules 2003, 36, 2256-2272.

[32] D. J. Keddie, G. Moad, E. Rizzardo, S. H. Thang, Macromolecules 2012, 45(13), 5321-5342.

[33] J. O. Zoppe, N. C. Ataman, P. Mocny, J. Wang, J. Moraes, H. A. Klok, Chemical Reviews 2017, 117(3), 1105-1318.

[34] C. Li, J. Han, C. Y. Ryu, B. C. Benicewicz, Macromolecules 2006, 39, $3175-3183$.

[35] D. Zhao, M. Di Nicola, M. M. Khani, J. Jestin, B. C. Benicewicz, S. K. Kumar, Soft Matter 2016, 12(34), 7241-7247.

[36] P. S. Chinthamanipeta, S. Kobukata, H. Nakata, D. A. Shipp, Polymer 2008, 49(26), 5636-5642.

[37] B. Ebeling, S. Eggers, M. Hendrich, A. Nitschke, P. Vana, Macromolecules 2014, 47(4).

[38] P. Brandani, P. Stroeve, Macromolecules 2004, 6640-6643.

[39] Y. Zhao, S. Perrier, Macromolecules 2007, 40(25), 9116-9124.

[40] R. Rotzoll, P. Vana, Journal of Polymer Science Part A: Polymer Chemistry 2008, 46(23), 7656-7666.

[41] D. H. Nguyen, P. Vana, Polymers for Advanced Technologies 2006, 17(9-10), 625-633. 
[42] R. Ranjan, R. Ranjan, W. J. Brittain, W. J. Brittain, Macromolecules 2007, 40(17), 6217-6223.

[43] Y. Tsujii, M. Ejaz, K. Sato, A. Goto, T. Fukuda, Macromolecules 2001, 34(26), 8872-8878.

[44] C. Li, B. C. Benicewicz, Macromolecules 2005, 38(14), 5929-5936.

[45] D. Zhou, E. Mastan, S. Zhu, Macromolecular Theory and Simulations 2012, 21(9), 602-614.

[46] W. Ring, I. Mita, A. D. Jenkins, N. M. Bikales, Pure and Applied Chemistry 1985, 57(10), 1427-1440.

[47] L. J. Young, T. Dow, Journal of Polymer Science 1961, 54, 411-455.

[48] F. R. Mayo, F. M. Lewis, Journal of the American Chemical Society 1944, 66(9), 1594-1601.

[49] F. M. Lewis, C. Walling, W. Cummings, E. R. Briggs, W. J. Wenisch, Journal of the American Chemical Society 1948, 70(4), 1527-1529.

[50] D. J. Keddie, Chemical Society Reviews 2014, 43(2), 496-505.

[51] M. D. Lechner, K. Gehrke, E. H. Nordmeier, Makromolekulare Chemie, Springer Spektrum, Berlin, 2014.

[52] R. W. Lunn, Industrial \& Engineering Chemistry 1939, 31(10), 11901192.

[53] K. Hübner, Chemie in unserer Zeit 2016, 50(1), 67-71.

[54] T. A. Vilgis, G. Heinrich, Physik Journal 2001, 57(6), 67-73.

[55] Continental Reifen Deutschland GmbH, Reifengrundlagen Pkw, 2009.

[56] S. S. Sternstein, S. Amanuel, M. L. Shofner, Rubber Chemistry and Technology 2010, 83(2), 181-198.

[57] M.-J. Wang, Rubber Chemistry and Technology 1998, 71(3), 520-589.

[58] S. Maghami, W. K. Dierkes, T. V. Tolpekina, S. M. Schultz, J. W. M. Noordermeer, Rubber Chemistry and Technology 2012, 85(4), 513525. 
[59] F. R. Schwarzl, F. Zahradnik, Rheologica Acta 1980, 19(2), 137-152.

[60] S. S. Choi, Polymers for Advanced Technologies 2002, 13(6), 466-474.

[61] I. Pliskin, N. Tokita, Journal of Applied Polymer Science 1972, 16(2), 473-492.

[62] C. G. Robertson, C. J. Lin, M. Rackaitis, C. M. Roland, Macromolecules 2008, 41, 2727-2731.

[63] E. M. Dannenberg, Rubber Chemistry and Technology 1986, 59(3), 512-524.

[64] C. G. Robertson, C. M. Roland, Rubber Chemistry and Technology 2008, 81(3), 506-522.

[65] C. G. Robertson, C. J. Lin, R. B. Bogoslovov, M. Rackaitis, P. Sadhukhan, J. D. Quinn, C. M. Roland, Rubber Chemistry and Technology 2011, 84(4), 507-519.

[66] S. Merabia, P. Sotta, D. R. Long, Macromolecules 2008, 41(21), 82528266.

[67] A. Nitschke, P. Vana, J. Davin, N. Hojdis, C. Recker, K. Herzog, Silica mit modifizierter Oberfläche 2019, under preparation.

[68] A. Ansarifar, L. Wang, R. J. Ellis, S. P. Kirtley, N. Riyazuddin, Journal of Applied Polymer Science 2007, 105(2), 322-332.

[69] H. S. Katz, J. Mileski, Handbook of Fillers for Plastics, Springer, Berlin, 1987.

[70] X. D. Wang, Z. X. Shen, T. Sang, X. B. Cheng, M. F. Li, L. Y. Chen, Z. S. Wang, Journal of Colloid and Interface Science 2010, 341(1), 23-29.

[71] S. S. Sarkawi, W. K. Dierkes, J. W. M. Noordermeer, Rubber Chemistry and Technology 2015, 88(3), 359-372.

[72] J.-W. Park, Y. J. Park, C.-H. Jun, Chemical Communications 2011, 47(17), 4860. 
[73] L. Wang, J. Li, Y. Zheng, Y. Huang, Y. Qiao, B. C. Benicewicz, in Controlled Radical Polymerization: Materials, (Eds.: J. C. Krzysztof Matyjaszewski, Brent S. Sumerlin, Nicolay V. Tsarevsky), American Chemical Society, ACS Symposium Series 1188, Cha. 13, 2015, 187201.

[74] W. Baumann, Kautschuck und Gummi, Springer, Heidelberg, 1998.

[75] R. Ebewele, Polymer Science and Technology, CRC Press, 2000.

[76] P. Froimowicz, B. Van Heukelum, C. Scholten, K. Greiner, O. Araujo, K. Landfester, Journal of Polymer Science, Part A: Polymer Chemistry 2014, 52(7), 883-889.

[77] P. Ganjeh-Anzabi, V. Haddadi-Asl, M. Salami-Kalajahi, M. Abdollahi, Journal of Polymer Research 2013, 20(9), 1-8.

[78] A. Nitschke, Synthese funktionalisierter Silica- und Ruß-Nanopartikel mittels RAFT-Polymerisation, Masterthesis, Georg-August-University Göttingen, 2015.

[79] C. Li, J. Han, C. Y. Ryu, B. C. Benicewicz, Macromolecules 2006, 39, 3175-3183.

[80] P. Vondráček, M. Hradec, V. Chvalovský, H. D. Khanh, Rubber Chemistry and Technology 1984, 57(4), 675-685.

[81] W. Shen, Q. Qiu, Y. Wang, M. Miao, B. Li, T. Zhang, A. Cao, Z. An, Macromolecular Rapid Communications 2010, 31(16), 1444-1448.

[82] B. Robak, J. Rogoża, M. Łapkowski, Journal of Elastomers and Plastics 2019, 51(3), 244-261.

[83] S. S. Choi, B. H. Park, C. Nah, Journal of Applied Polymer Science 2003, 90(11), 3135-3140.

[84] S. S. Choi, B. H. Park, H. Song, Polymers for Advanced Technologies 2004, 15(3), 122-127.

[85] S. J. Park, K. S. Cho, Journal of Colloid and Interface Science 2003, 267(1), 86-91.

[86] Y. Zhou, L. Yongli, Y. Huang, Micro- and Macromechanical Properties of Materials, CRC Press, Boca Raton, 2014. 
[87] W. K. Dierkes, L. A. E. M. Reuvekamp, J. W. M. Ten Brinke, A. J. W. Noordermeer, in Silanes and Other Coupling Agents, Volume 3, (Eds.: L. K. Mittal), CRC Press, Boca Raton, 2004, 89-103.

[88] L. Brinkmann, Synthese zur Funktionalisierung von Carbon Black zur Modifizierung der mechanischen Eigenschaften von PolymerNanokompositen, Bachelorthesis, Georg-August-University Göttingen, 2018.

[89] A. Nitschke, L. Riemann, L. Kollenbach, V. Braun, M. Buback, P. Vana, Macromolecular Chemistry and Physics 2020, 221 (1), 1900345.

[90] P. M. Visakh, S. Thomas, A. K. Chandra, A. P. Mathew, Advances in Elastomers I, Springer, Berlin, Heidelberg, 2013.

[91] J.-B. Donnet, R. C. Bansal, M.-J. Wang, Carbon Black: Sciene and Technology, Second Edition, Taylor \& Francis, New York, 1993.

[92] J. C. Salamone, Polymeric Materials Encyclopedia, Springer, Heidelberg, 2013.

[93] J. Kuhn, Physik in Unserer Zeit 1992, (2), 84-89.

[94] L. K. R. Dittmeyer, W. Keim, G. Kreysa, A. Oberholz, K. Winnacker, Chemische Technik, Band 7: Industriprodukte, Wiley-VCH Verlag, Weinheim, 2004.

[95] M. L. Studebaker, Rubber Chemistry and Technology 1957, 30(5), $1400-1483$.

[96] I. Kumar, S. Rana, J. W. Cho, Chemistry - A European Journal 2011, 17(40), 11092-11101.

[97] N. B. Pramanik, N. K. Singha, RSC Advances 2015, 5(114), 9432194327.

[98] Q. Yang, L. Wang, W. Xiang, J. Zhou, Q. Tan, Polymer 2007, 48(12), 3444-3451.

[99] T. Liu, S. Jia, T. Kowalewski, K. Matyjaszewski, R. Casado-Portilla, J. Belmont, Langmuir 2003, 19(16), 6342-6345.

[100] N. Roy, R. Sengupta, A. K. Bhowmick, Progress in Polymer Science 2012, 37(6), 781-819. 
[101] T. Xu, Z. Jia, J. Li, Y. Luo, D. Jia, Z. Peng, Polymer Composites 2018, 39(2), 377-385.

[102] F. Morales-Lara, M. Domingo-García, R. López-Garzón, M. Luz Godino-Salido, A. Peñas-Sanjuán, F. J. López-Garzón, M. PérezMendoza, M. Melguizo, Science and Technology of Advanced Materials 2016, 17(1), 541-553.

[103] P. Petrov, F. Stassin, C. Pagnoulle, R. Jérôme, Chem. Commun. 2003, (23), 2904-2905.

[104] S.-J. Park, K.-S. Cho, S.-K. Ryu, Carbon 2003, 41 (7), 1437-1442.

[105] F. Beckert, C. Friedrich, R. Thomann, R. Mülhaupt, Macromolecules 2012, 45(17), 7083-7090.

[106] H. He, M. Zhong, D. Konkolewicz, K. Yacatto, T. Rappold, G. Sugar, N. E. David, K. Matyjaszewski, Journal of Materials Chemistry A 2013, 1(23), 6810-6821.

[107] C. Goussé, A. Gandini, P. Hodge, Macromolecules 1998, 31 (2), 314 321.

[108] Q. Jiang, D. Jia, J. Yang, J. Zhong, Polymer - Plastics Technology and Engineering 2007, 46(12), 1167-1171.

[109] W. F. Brucksch, Rubber Chemistry and Technology 1963, 36(4), 975987.

[110] A. Ma, J. Zhang, N. Wang, L. Bai, H. Chen, W. Wang, H. Yang, L. Yang, Y. Niu, D. Wei, Industrial and Engineering Chemistry Research 2018, 57(51), 17417-17429.

[111] S. Dumitrescu, M. Grigorq, C. I. Simionescu, Macromolecular Chemistry and Physics 1983, 2040, 2033-2040.

[112] D. Boschmann, P. Vana, Macromolecules 2007, 40(8), 2683-2693.

[113] C. I. Simionescu, M. Grigoras, V. Bărboiu, Journal of Polymer Science: Polymer Chemistry Edition 1985, 23(8), 2089-2098.

[114] F. Fleischhaker, A. P. Haehnel, A. M. Misske, M. Blanchot, S. Haremza, C. Barner-Kowollik, Macromolecular Chemistry and Physics 2014, 215(12), 1192-1200. 
[115] I. Aqeel, S; Lath, Dieter; Lathová, Elena; Pavlinec, Juraj; Lacík, Polymer bulletin 2002, 47(6), 563-569.

[116] M. Beiner, K. Schröter, E. Hempel, S. Reissig, E. Donth, Macromolecules 1999, 32(19), 6278-6282.

[117] T. Gruendling, T. Junkers, M. Guilhaus, C. Barner-Kowollik, Macromolecular Chemistry and Physics 2010, 211(5), 520-528.

[118] M. Morris, Journal of Chromatography A 1971, 55(1), 203-210.

[119] A. N. Nikitin, I. Laclk, R. A. Hutchinson, M. Buback, G. T. Russell, Macromolecules 2019, 52(1), 55-71.

[120] P. Drawe, M. Buback, Macromolecular Theory and Simulations 2016, 25(1), 74-84.

[121] H. Kattner, M. Buback, Macromolecular Symposia 2013, 333(1), 1123.

[122] S. Beuermann, M. Buback, T. P. Davis, R. G. Gilbert, R. A. Hutchinson, A. Kajiware, B. Klumperman, G. T. Russell, Macromolecular Chemistry and Physics 2000, 201(12), 1355-1364.

[123] S. Beuermann, M. Buback, T. P. Davis, R. G. Gilbert, R. A. Hutchinson, O. F. Olaj, G. T. Russell, J. Schweer, A. M. Van Herk, Macromolecular Chemistry and Physics 1997, 198(5), 1545-1560.

[124] G. Evan Roberts, T. P. Davis, J. P. Heuts, G. E. Ball, Macromolecules 2002, 35(27), 9954-9963.

[125] S. Beuermann, N. García, Macromolecules 2004, 37(8), 3018-3025.

[126] L. Couvreur, G. Piteau, P. Castignolles, M. Tonge, B. Coutin, B. Charleux, J. P. Vairon, Macromolecular Symposia 2001, 174, 197207.

[127] M. Buback, Macromolecular Symposia 2009, 275-276(1), 90-101.

[128] M. Buback, Macromolecular Rapid Communications 2015, 36(22), 1979-1983. 
[129] A. P. Haehnel, K. Kockler, T. Bantle, C. Barner-Kowollik, B. Wenn, T. Junkers, A. M. Misske, F. Fleischhaker, Macromolecular Rapid Communications 2014, 35(23), 2029-2037.

[130] G. Ovejero, M. D. Romero, E. Díez, I. Díaz, European Polymer Journal 2010, 46(12), 2261-2268.

[131] J. O. Weston, H. Miyamura, T. Yasukawa, D. Sutarma, C. A. Baker, P. K. Singh, M. Bravo-Sanchez, N. Sano, P. J. Cumpson, Y. Ryabenkova, S. Kobayashi, M. Conte, Catalysis Science and Technology 2017, 7(18), 3985-3998.

[132] B. Y. Chong, T. P. Le, G. Moad, E. Rizzardo, S. H. Thang, Macromolecules 1999, 32(6), 2071-2074. 